\title{
CHARACTERIZATION OF THE HEMODYNAMIC RESPONSES OF THE
} HIPPOCAMPAL AND PARAHIPPOCAMPAL REGIONS USING FMRI

\author{
by \\ Priyanka Mehta \\ Bachelor of Biomedical Engineering, University of Mumbai, 2013 \\ Mumbai, India
}

A thesis presented to Ryerson University

in partial fulfillment of the

requirements for the degree of

Master of Science

in the Program of

Biomedical Physics

Toronto, Ontario, Canada, 2016

(C) Priyanka Mehta 2016 


\section{Author's Declaration}

\section{AUTHOR'S DECLARATION}

I hereby declare that I am the sole author of this thesis. This is a true copy of the thesis, including any required final revisions, as accepted by my examiners. I authorize Ryerson University to lend this thesis to other institutions or individuals for the purpose of scholarly research. I further authorize Ryerson University to reproduce this thesis by photocopying or by other means, in total or in part, at the request of other institutions or individuals for the purpose of scholarly research. I understand that my thesis may be made electronically available to the public.

Priyanka Mehta 


\title{
Abstract
}

\section{Characterization of the Hemodynamic Responses of the Hippocampal and Parahippocampal Regions using fMRI}

Priyanka Mehta

\author{
Master of Science, Biomedical Physics
}

Ryerson University, 2016

Previous neuroimaging studies have suggested a dominant role of the right medial temporal lobe (MTL) structures- the hippocampal and parahippocampal regions in spatial memory processing. However, the underlying physiological hemodynamic response functions (HRF) of the MTL substructures remain undefined. Given the neuroanatomical differences between these substructures, it is posited that their hemodynamic characteristics are distinct. In this study, the hemodynamic responses of the MTL substructures are investigated using an optimization algorithm that penalizes the curvature (i.e. second derivative) of HRF. The time-to-peak characteristic of the hemodynamic responses revealed that the right CA3 and DG subfields of the hippocampus are significantly more active than the right CA1 subfield during a specific spatial memory task. Further, the hemodynamic responses of the entorhinal, perirhinal and parahippocampal cortices are presented. Together, these findings may help advance our understanding of neurodegenerative diseases like epilepsy and Alzheimer's disease that are strongly associated to hippocampal dysfunction. 


\section{Acknowledgements}

I would like to seize the opportunity to thank all the people who have directly or indirectly contributed to the accomplishment of this thesis over the past two years. Firstly, I wish to express my sincere gratitude and appreciation to my supervisors Dr. Dietmar Cordes and Dr. Michael Kolios. Dr. Cordes' incredible intuition and insight lie behind many of the ideas in this work. I am particularly indebted to him for providing me with the intellectual freedom, at the same time continuing to push me to reach beyond my limits. I am also very grateful to have had the opportunity to work with Dr. Kolios. This thesis would not have been possible without his persistent guidance and invaluable support.

I would like to thank the members of my supervisory committee Dr. Yuan Xu and Dr. Jahan Tavakkoli. I greatly appreciate their time in providing valuable feedback and the substantial influence it has had on my research. My heartfelt thanks also go to the Program Director, Dr. Carl Kumaradas for his unsurpassed encouragement time and again. I would like to commend the staff at the Ryerson University's Department of Physics for the extremely friendly research environment they maintain. I would also like to recognize my long-distance colleague at the Lou Ruvo Center for Brain Health in Las Vegas, Xiaowei Zhuang for her constant assistance throughout the past few months. Furthermore, I gratefully acknowledge the funding

received from the Natural Sciences and Engineering Research Council of Canada that made my Masters work possible.

Lastly and most importantly, I would like to credit my parents Hitesh \& Dipti Mehta, and my brother Parth Mehta for their love, support and encouragement in all my pursuits. I dedicate this thesis to them.

Priyanka Mehta 


\section{Table of Contents}

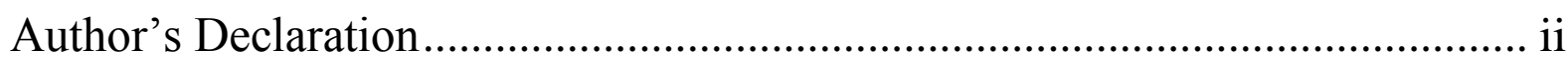

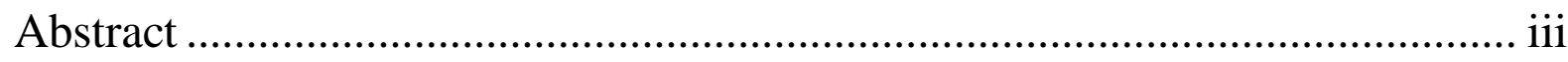

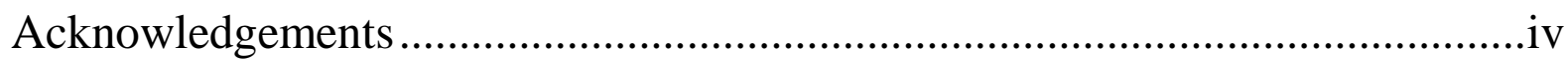

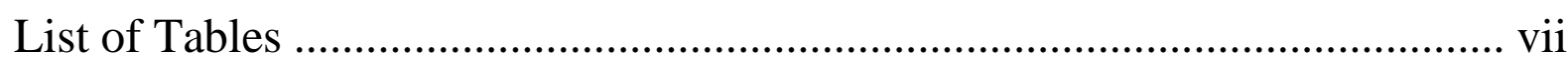

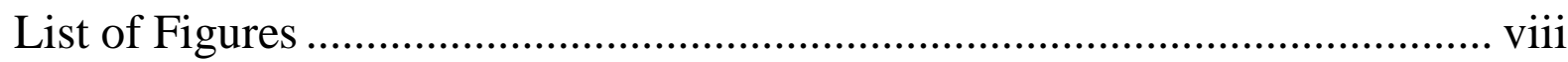

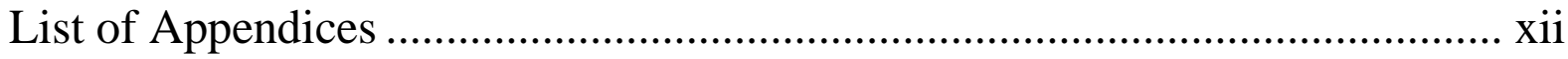

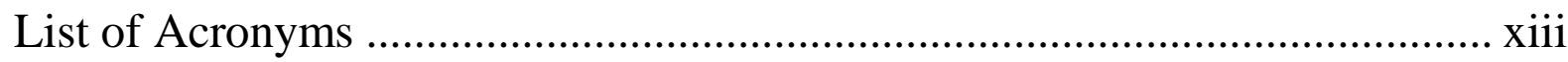

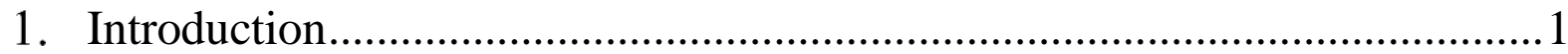

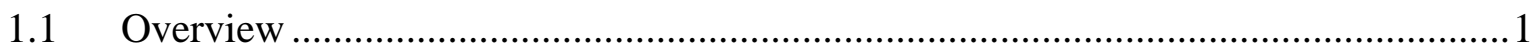

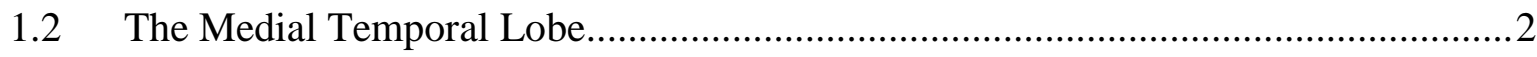

1.2.1 Neural Pathways ................................................................................................

1.2.2 Role in Spatial Memory.....................................................................................

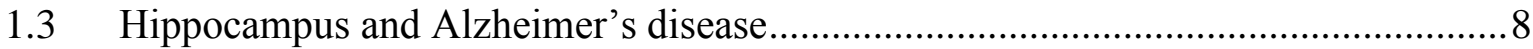

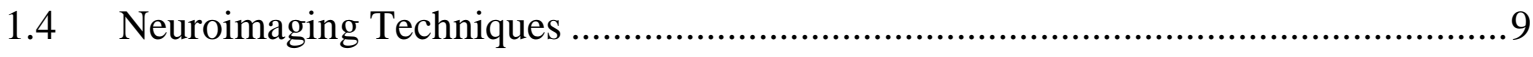

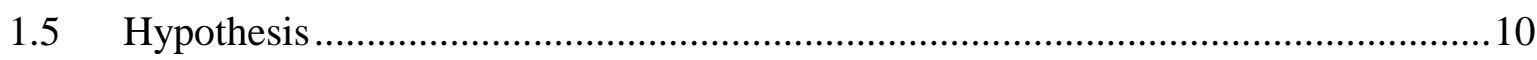

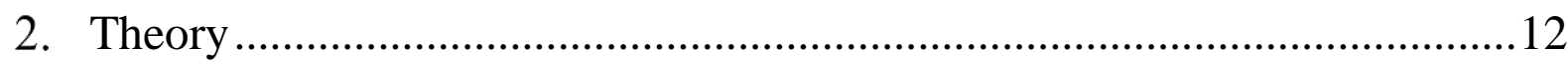

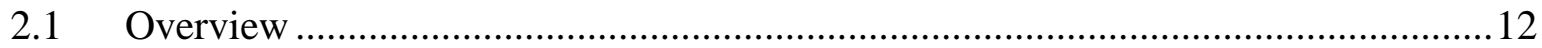

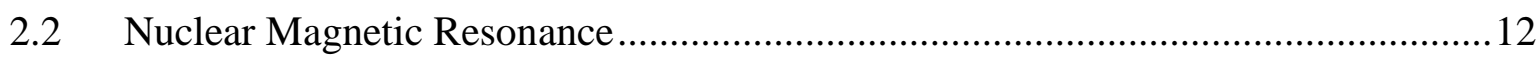

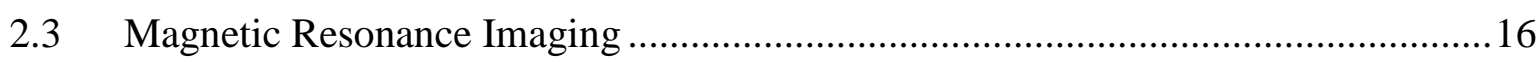

2.4 Functional Magnetic Resonance Imaging ………….............................................. 17

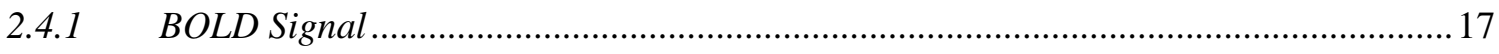

2.4.2 Hemodynamic Response Function ........................................................................ 19

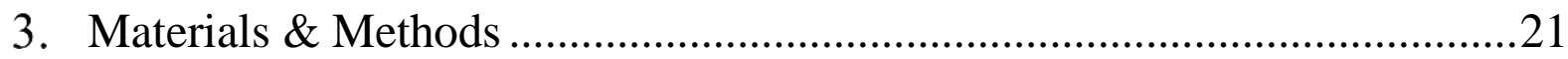

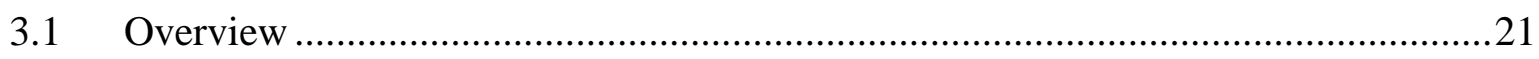

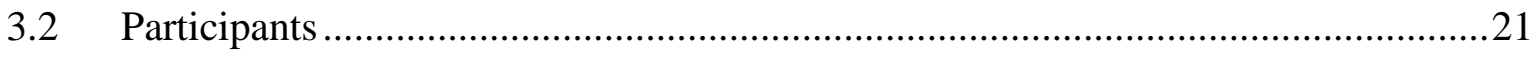

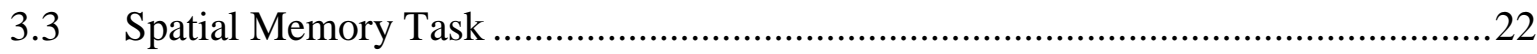




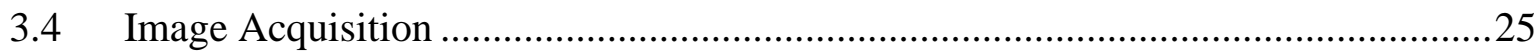

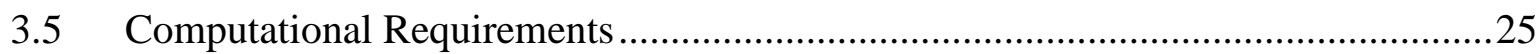

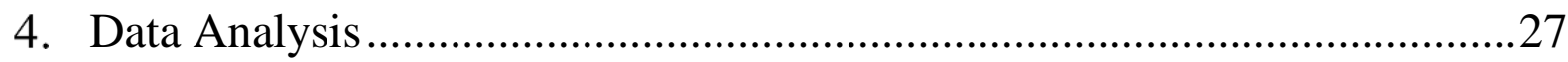

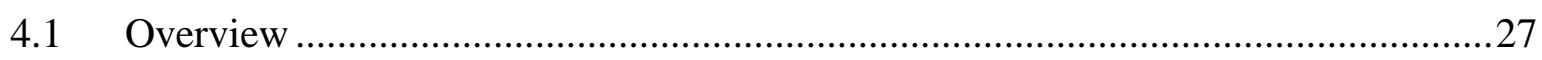

4.2 Independent Components Analysis ................................................................28

4.2.1 Probabilistic Independent Components Analysis.........................................................29

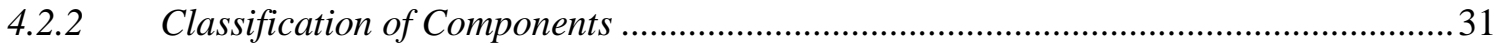

4.3 Cortical Reconstruction and Hippocampal Subfield Segmentation ..........................32

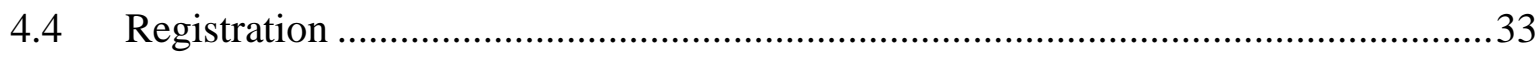

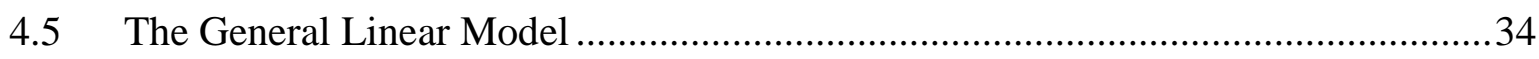

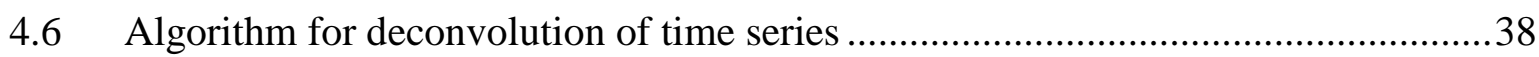

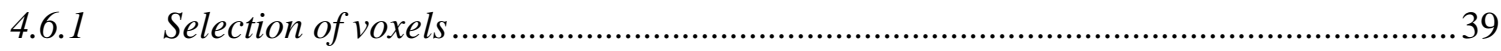

4.6.2 Hemodynamic response function model ...................................................................... 40

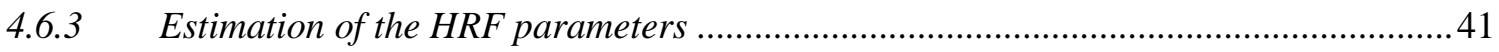

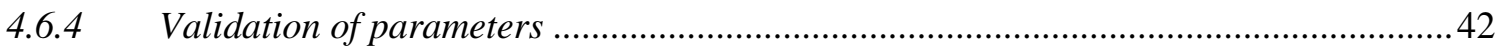

4.6.5 Determining the best parameters .............................................................................. 42

5. Results............................................................................44

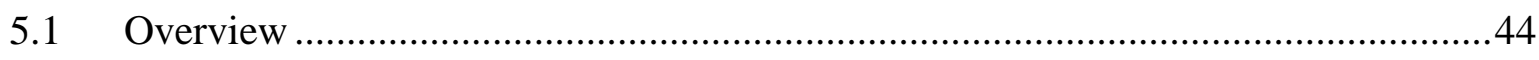

5.2 Independent Components Analysis Results ...................................................44

5.3 Hippocampal Formation Segmentation...................................................... 47

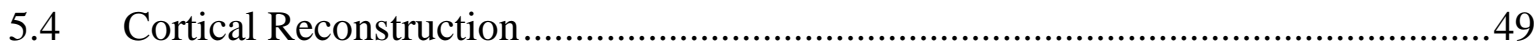

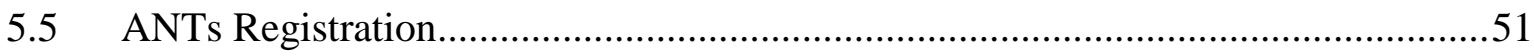

5.6 Hemodynamic Response Functions ............................................................52

6. Discussions \& Conclusions ..........................................................62 62

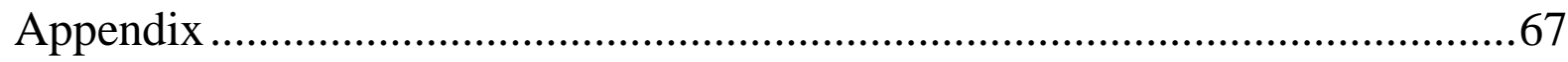

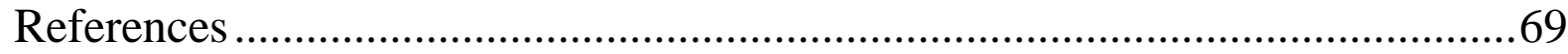




\section{List of Tables}

Table 2.1: Typical $T_{1}$ and $T_{2}$ values for grey \& white matter at 3.0 T magnetic field (Wansapura et al., 1999)... 16

Table 3.1: Demographics of the participants...... .22

Table 3.2: Description of stimuli conditions presented for the spatial memory task .......................23

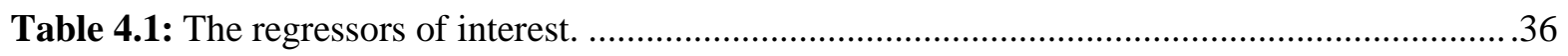

Table 4.2: Number of voxels recorded within each sub-region averaged across all the participants and their corresponding standard deviations.

Table 5.1: Summary of time-to-peak (in seconds) for the left and right sub-regions and their associated propagated uncertainties calculated using the Equation 5.1. 


\section{List of Figures}

Figure 1.1: Anatomy of the Medial temporal lobe. (a) A coronal slice of a weighted structural MR image through the medial temporal lobe region, as indicated by the box. (b) MTL sub-structures displayed on the magnified structural image. 3

Figure 1.2: (a) Model of the hippocampal formation depicting the trisynaptic loop (not to scale). EC $\rightarrow$ DG via the perforant pathway (synapse 1 ), DG $\rightarrow$ CA3 via mossy fibres (synapse 2), CA3 $\rightarrow$ CA1 via Schaffer collaterals (synapse 3). (b) Simplified circuit diagram of neuroanatomical connectivity of the MTL region reflecting dominant pathways.

Figure 1.3: Place responsive cells. Letters $S_{A}, S_{B}, S_{C}$ indicate shop locations, white boxes indicate nontarget buildings and the red line indicates the participant's trajectory. (a) Firing rate (in $\mathrm{Hz}$ ) of a right hippocampal cell at various spatial locations. (b) Comparison of place responsive cells in the hippocampus (H), parahippocampal region (PR), amygdala (A) and the frontal lobe (FR). (Courtesy: Ekstrom et al., 2003)

Figure 1.4: (a) Task design for the object task (left) and the spatial task (right). (b) Comparison of the activations in the perirhinal, anterior parahippocampal $(\mathrm{PH})$ cortex and posterior parahippocampal (PH) cortex for the Object task and Spatial Task. (Courtesy: Buffalo, Bellgowan and Martin, 2006)

Figure 1.5: The generation of a blood oxygenation level dependent (BOLD) signal in fMRI. .10

Figure 2.1: Behaviour of a sample when placed in a strong magnetic field. (a) The nuclear magnetic moments are randomly oriented when no external field is applied. (b) When an external magnetic field is applied the moments align either parallel to the field or anti-parallel. (c) The slight preferential alignment parallel to the field gives rise to a net magnetization vector $M$. (d) When an RF pulse is applied, the magnetization vector changes its orientation from the $z$-axis into the $x y$-plane. Due to the non-uniformity of static magnetic field within each voxel and spin-spin interactions, the transverse magnetization vector decays exponentially with time constant $T_{2}^{*}$.

Figure 2.2: Examples of MRI scans. (a) $T_{1}$ - weighted anatomical scan. (b) $T_{2}$ - weighted anatomical scan. (c) $T_{2}^{*}$ - weighted functional scan.

Figure 2.3: (a) In a baseline state the neurons require a certain amount of oxygen. (b) When neuronal activity increases, the demand for also increases thereby increasing the blood flow. This results in a 
sudden increase in the blood oxygen concentration in an active state compared to the baseline state. .18

Figure 2.4: The experimental paradigm passes through the brain dynamics and the MRI scanner before being measured as an fMRI signal. .19

Figure 2.5: Schematic representation of a typical hemodynamic response function. .20

Figure 3.1: Design of the fMRI spatial memory task. (a) Example of stimuli interleaved with baseline task. (b) A total of $n=450$ stimuli were displayed. Each stimulus was displayed for $3 \mathrm{~s}$ followed by a 1s blank/rest period.

Figure 3.2: (a) Schematic of the fMRI experiment setup. (b) A total of 2380 volumes of $T_{2}^{*}$-weighted scans were acquired every $0.765 \mathrm{~s}$. .26

Figure 4.1: Independent Components Analysis- An fMRI spatio-temporal dataset is expressed as a linear combination of independent spatial maps and its associated time courses. .29

Figure 4.2: Two-step process for registration of the subfields to the functional scan. (a) The anatomical $T_{1}$ - weighted image is registered to the mean functional image $T_{2}^{*}$. This process produces a transformation matrix. (b) The transformation matrix can be applied to the hippocampal subfield segmentation obtained from FreeSurfer since the segmentation is derived from the $T_{1}$ - weighted image.

Figure 4.3: Modelling the BOLD response. (a) The reference function is specified as a box-car function with ' 1 ' indicating the 'ON' condition and ' 0 ' indicating the 'OFF' condition. This function is convolved with (b) the hemodynamic response function to produce (c) the expected BOLD signal.

Figure 4.4: Flowchart of the algorithm. .43

Figure 5.1: Example of spatial component maps generated by ICA. (a) Component 1: Noise (b) Component 2: Signal. .45

Figure 5.2: The power spectrum and time course associated with the spatial maps. (a) Component 1: Noise. (b) Component 2: Signal. .46

Figure 5.3: Segmented hippocampal formation for participants 1 through 4 overlaid on a $T_{1}$ - weighted MR image. Blue, red, green and cyan colors indicate the subiculum, CA1, CA3 and dentate gyrus subfields respectively.

Figure 5.4: Segmented hippocampal formation for participants 5 through 7 overlaid on a $T_{1}$-weighted MR image. Blue, red, green and cyan colors indicate the subiculum, CA1, CA3 and dentate gyrus subfields respectively. 
Figure 5.5: Cortical labels for participants 1 through 4, overlaid on the left (left panel) and right (right panel) inflated hemispheres. Yellow, blue and magenta colors indicate the parahippocampal, entorhinal and perirhinal cortices respectively.

Figure 5.6: Cortical labels for participants 5 through 7, overlaid on the left (left panel) and right (right panel) inflated hemispheres. Yellow, blue and magenta colors indicate the parahippocampal, entorhinal and perirhinal cortices respectively. .50

Figure 5.7: The hippocampal subfields after registration to the mean functional scan $T_{2}^{*}$ as seen in the (a) axial (b) sagittal and (c) coronal slices. .51

Figure 5.8: Result for the validation step of one of the voxels. The best HRF is obtained at the minimum of the objective function $(\mu=4.5)$. The graph depicts how the functional form of the HRF changes with $\mu$. Three hemodynamic response functions are shown above the graph corresponding to $\mu=2.7$ (small figure $A$ ), $\mu=4.5$ (small figure $B$ ) and $\mu=7.1$ (small figure $C$ ). .53

Figure 5.9: The physiological hemodynamic response functions averaged across all voxels within a sub-region from both runs for participant \#1. (a) HRF for right medial temporal lobe structures. (b) HRF for left medial temporal lobe structures. (c) Comparison of the average time-to-peak of the HRF and error bars representing its associated standard deviation.

Figure 5.10: The physiological hemodynamic response functions averaged across all voxels within a sub-region from both runs for participant \#2. (a) HRF for right medial temporal lobe structures. (b) HRF for left medial temporal lobe structures. (c) Comparison of the average time-to-peak of the HRF and error bars representing its associated standard deviation. .55

Figure 5.11: The physiological hemodynamic response functions averaged across all voxels within a sub-region from both runs for participant \#3. (a) HRF for right medial temporal lobe structures. (b) HRF for left medial temporal lobe structures. (c) Comparison of the average time-to-peak of the HRF and error bars representing its associated standard deviation. .56

Figure 5.12: The physiological hemodynamic response functions averaged across all voxels within a sub-region from both runs for participant \#4. (a) HRF for right medial temporal lobe structures. (b) HRF for left medial temporal lobe structures. (c) Comparison of the average time-to-peak of the HRF and error bars representing its associated standard deviation. .57

Figure 5.13: The physiological hemodynamic response functions averaged across all voxels within a sub-region from both runs for participant \#5. (a) HRF for right medial temporal lobe structures. (b) HRF for left medial temporal lobe structures. (c) Comparison of the average time-to-peak of the HRF and error bars representing its associated standard deviation. .58

Figure 5.14: The physiological hemodynamic response functions averaged across all voxels within a sub-region from both runs for participant \#6. (a) HRF for right medial temporal lobe structures. (b) 
HRF for left medial temporal lobe structures. (c) Comparison of the average time-to-peak of the HRF and error bars representing its associated standard deviation.

Figure 5.15: The physiological hemodynamic response functions averaged across all voxels within a sub-region from both runs for participant \#7. (a) HRF for right medial temporal lobe structures. (b) HRF for left medial temporal lobe structures. (c) Comparison of the average time-to-peak of the HRF and error bars representing its associated standard deviation. 


\section{List of Appendices}

Appendix A: The accuracy (percent correct responses) and response time (in seconds) 


\section{List of Acronyms}

MTL Medial Temporal Lobe

BOLD Blood Oxygen Level Dependent

fMRI Functional Magnetic Resonance Imaging

HRF Hemodynamic Response Function

GLM General Linear Model

ICA Independent Component Analysis

MRI Magnetic Resonance Imaging

NMR Nuclear Magnetic Resonance

$\mathrm{Hb} \quad$ Haemoglobin

AD Alzheimer's Disease 


\section{Introduction}

\subsection{Overview}

The hippocampus, embedded in the medial temporal lobe of each hemisphere is one of the most comprehensively studied areas of the brain because of its distinctive structure and fundamental role in memory. The large, bulging structure of the hippocampus resembling the horns of a ram impressed ancient anatomists. The advent of a histological technique, now called the Golgi method developed by Camillo Golgi provided the first big advancement in unravelling the cellular organization of the hippocampus. The principal cells in the hippocampus are arranged in a single layer, a layout very different from other neocortical areas. This finding further reinforced the interest in the hippocampal investigations. Early research suggested the possible role of the hippocampus in olfactory (Ferrier, 1876), emotion (Papez, 1937) and attention control (Sloan and Jasper, 1950) functions. However, the pivotal role of the hippocampus in memory became evident in the early 1950s when Henry Molaison, famously known as Patient H.M., suffered a pure memory deficit after a bilateral resection of the medial temporal lobe for relief of epilepsy (Scoville and Milner, 1957). Ever since, numerous studies have been conducted in an effort to fathom the function of the hippocampus and the medial temporal lobe structures in general.

In this thesis, the function of the medial temporal lobe structures as related to spatial memory are examined. The hippocampus proper and entorhinal cortex collectively referred to as the hippocampal formation and the perirhinal and parahippocampal cortex collectively referred to as the parahippocampal regions. Specifically, the hemodynamics of the medial temporal lobe structures in response to a spatial memory task are investigated. In so doing, MRI brain scans of the participants are acquired while they perform a spatial memory task. These scans are analysed using an optimization algorithm proposed in this 
thesis to compute the hemodynamic responses of the regions of interest- hippocampal and parahippocampal regions in this case.

This thesis is organized as follows: The relevant literature review describing the roles of the medial temporal lobe structures in spatial memory are given in this Chapter. The objectives and significance of this study are also stated. The principles of Magnetic Resonance Imaging and functional Magnetic Resonance Imaging are provided in Chapter 2. Additionally, the anatomy of the hippocampus and the surrounding parahippocampal regions and their relation to spatial memory processing are also detailed. The experimental methods used for this study are described in Chapter 3. In particular, the spatial memory task, image acquisition techniques and participant data for this study are discussed. In Chapter 4, the exploratory data analysis technique used for preprocessing in this study- Independent Components Analysis is introduced. An algorithm for deconvolution of the physiological hemodynamic response functions from the acquired fMRI time series is also proposed. The results of the Independent Components Analysis and the hemodynamic responses from the regions that are of interest are presented in Chapter 5. The main findings are discussed in Chapter 6. A list of frequently used list acronyms are given in Appendix A.

\subsection{The Medial Temporal Lobe}

The hippocampal formation along with the parahippocampal regions form a part of the medial temporal lobe (MTL) memory system of the human brain. Deep within the temporal lobe of the human brain as seen in Figure 1.1 (a), on its medial surface lies a group of many millions of neurons called the hippocampal formation. The hippocampal formation measures up to $4.5-5 \mathrm{~cm}$ and comprises of the hippocampus proper, dentate gyrus, subicular complex and entorhinal cortex as seen in Figure 1.1 (b). The hippocampus proper is further divided into three main subdivisions: CA1, CA2, and CA3. CA stands for 'Cornu Ammonis', named after the ancient Egyptian god Amun Kneph. Curled tightly around the CA3 
subfield is a V-shaped or U-shaped structure called the Dentate Gyrus (DG). Positioned between the hippocampus proper and entorhinal cortex and a range of cortical and subcortical structures is the subicular complex. The subicular complex includes the subiculum, presubiculum and parasubiculum regions. The entorhinal cortex is surrounded by a number of cortical areas. Medially, the entorhinal cortex fuses with structures that belong either to the hippocampal formation or the parahippocampal region, such as the amygdalo-hippocampal transition, and the parasubiculum. The lateral and posterior borders are with the other two major constituents of the parahippocampal region, the perirhinal cortex laterally and the parahippocampal cortex posteriorly. Throughout this thesis, the term 'hippocampus' is used to refer to the CA1, CA3, DG and subiculum collectively, the term 'hippocampal formation' is used to refer to the CA1, CA3, DG, subiculum and entorhinal cortex collectively while 'parahippocampal region' is used to refer to the perirhinal cortex and parahippocampal cortex collectively.

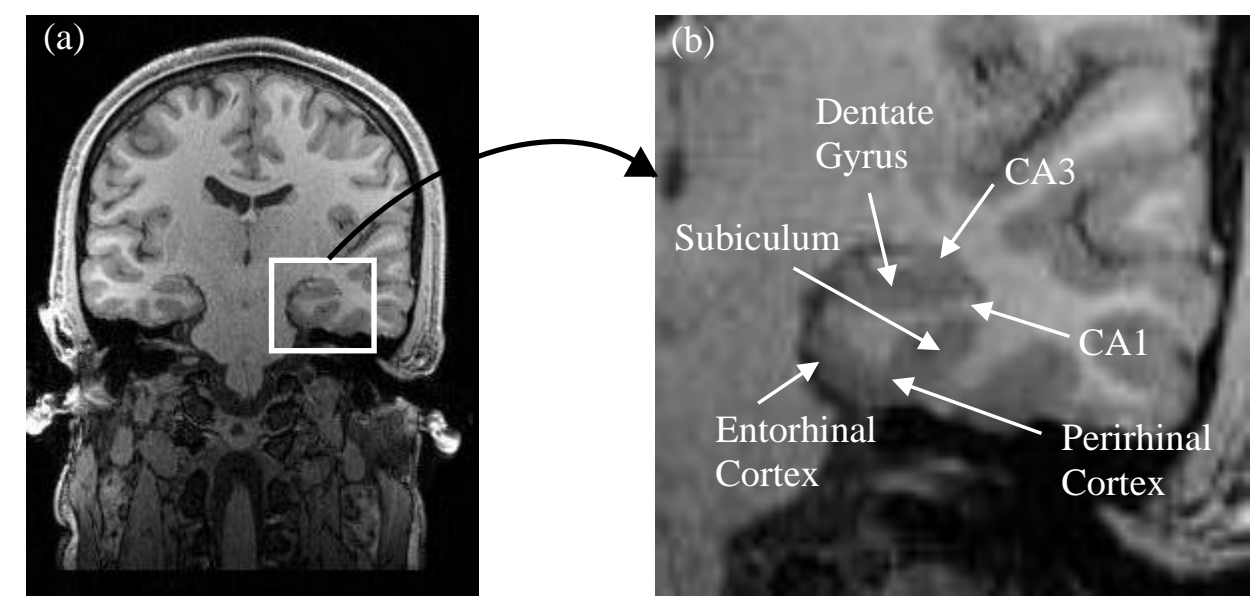

Figure 1.1: Anatomy of the Medial temporal lobe. (a) A coronal slice of a weighted structural MR image through the medial temporal lobe region, as indicated by the box. (b) MTL sub-structures displayed on the magnified structural image. 


\subsubsection{Neural Pathways}

The hippocampus forms a primarily unidirectional network with the entorhinal cortex (Broadmann Area 28). The entorhinal cortex (EC) is the neocortical nodal point sending projections into the hippocampus and communicating its output to other neocortical structures. As described by Ramon y Cajal $(1893,1911)$ and reproduced here in Figure. 1.2 (a), cells in the superficial layer (layer II) of the EC project their output to granule cells of DG and pyramidal cells of CA3 via the perforant pathway. DG granule cells project to CA3 region via mossy fiber pathway. In turn, projections from CA3 pyramidal cells include collaterals to other CA3 pyramidal cells comprising an extensive system of associational connections within the region. Projections from CA3 also include the Schaffer collaterals which constitute the major projection to CA1 pyramidal cells. CA1 receives additional input from EC (layer III) as part of direct pathway (monosynaptic). CA1 then projects to both the subiculum and deeper layers of EC. Unlike CA3 field, there are few associational connections within CA1.

As seen in Figure. 1.2 (b) the EC receives most of its highly processed neocortical input from the perirhinal and parahippocampal cortices which in turn is conveyed to the hippocampal formation. Perirhinal cortex projects primarily to the anterior two-thirds of EC while parahippocampal cortical projections terminate primarily in the posterior third. The Entorhinal Cortex also relays output from the subiculum of the hippocampal formation to the different neocortical regions. The EC therefore acts as an interface.

\subsubsection{Role in Spatial Memory}

The function of the hippocampus has long been implicated in two major memory forms: episodic memory - memory of past personally experienced events that can be explicitly stated and spatial memoryformation of cognitive maps and their use in navigation through space. The discovery of 'place cells' in 

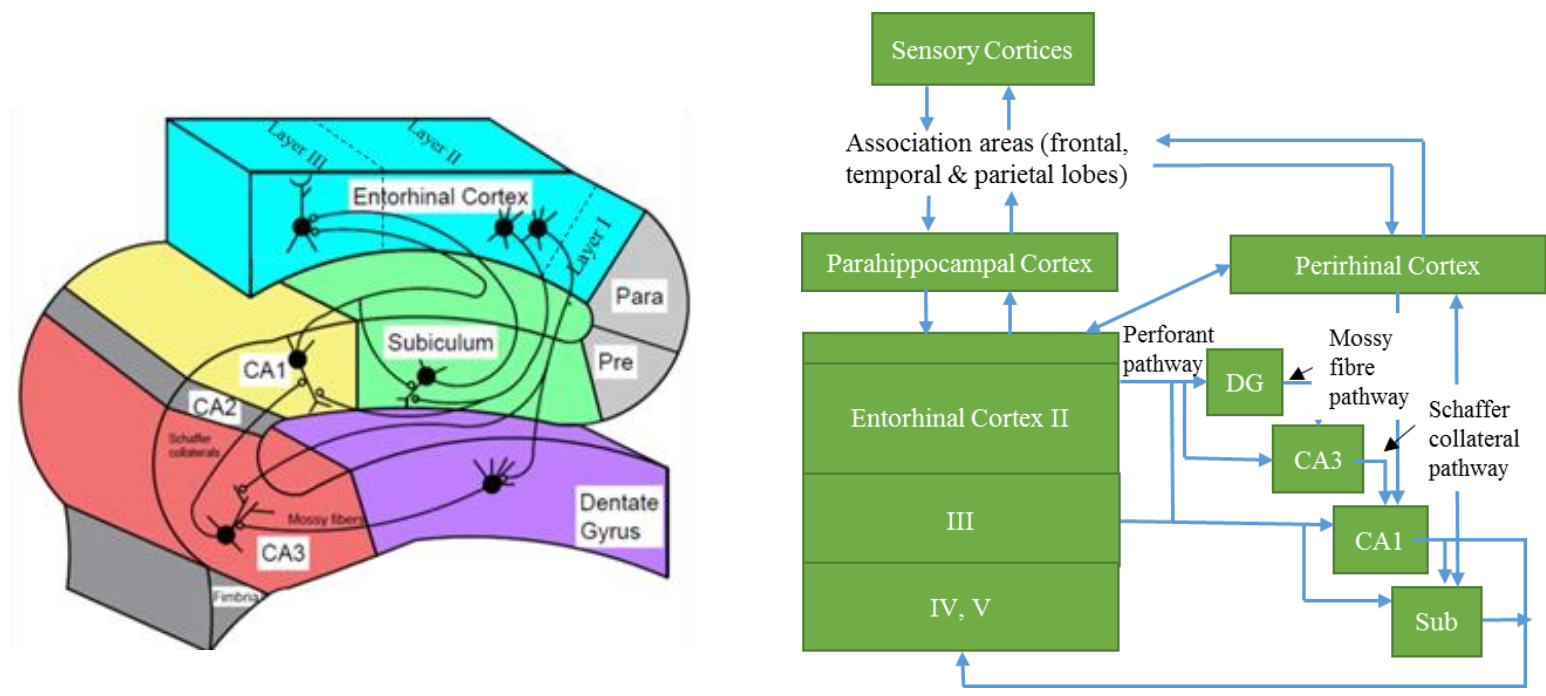

Figure 1.2: (a) Model of the hippocampal formation depicting the trisynaptic loop (not to scale). EC $\rightarrow$ DG via the perforant pathway (synapse 1 ), DG $\rightarrow$ CA3 via mossy fibres (synapse 2), CA3 $\rightarrow$ CA1 via Schaffer collaterals (synapse 3). (b) Simplified circuit diagram of neuroanatomical connectivity of the MTL region reflecting dominant pathways.

the hippocampus of freely moving rats by O'Keefe and Dostrovsky in 1971 motivated a number of studies in spatial memory. The Cognitive Map Theory (O'Keefe and Nadel, 1978) postulated that the place cellsencode a cognitive representation of a specific location in space to create a cognitive map. These cells fire when an animal traverses a specific location in its environment also known as the 'place field'. About a decade later, 'head-direction cells' that encode the orientation of the head in space were reported in the dorsal presubiculum of the hippocampal formation (Taube, Muller and Ranck, 1990). Soon after, 'grid cells' that provide information about distances traveled in a particular direction were discovered in a structure that is fused to the hippocampus called the medial entorhinal cortex (Hafting et al., 2005). The discovery of these cells in animals led to the origination of the idea that medial temporal lobe structures may likely play a central role in spatial memory in humans as well.

A study conducted by placing electrodes in the brain for recording single unit activity was conducted by Ekstrom and colleagues in 2003 . This study involved recording of data from electrodes implanted in 
the cells of the hippocampus, parahippocampal region, amygdala and frontal lobe while participants pretended to be taxi drivers navigating through a virtual town. It was noted that cells that respond to specific spatial locations are found in the hippocampus (Ekstrom et al., 2003). As seen in Figure 1.1 (a), the firing rate of a right hippocampal cell increases in frequently traversed spatial locations as compared to other locations. The places responsive cells were found to be clustered in the hippocampus Figure 1.1 (b).

The entorhinal cortex acts as an interface between the hippocampus and the neocortical regions. Recent fMRI data provided evidence for presence of grid-like cells which have been identified in rats in the entorhinal cortex of human participants navigating through a virtual reality arena (Doeller, Barry and Burgess, 2010). This study further showed that the hemodynamic activity in the entorhinal cortex is modulated by the direction of movement. Direct recordings from electrodes implanted in the entorhinal cortex further established its role in spatial memory (Jacobs et al., 2013). The locations at which increased firing rates of the cells were recorded were arranged in a triangular grid, similar to patterns observed in rodents (Hafting et al., 2005; Sargolini et al., 2006).

(a)

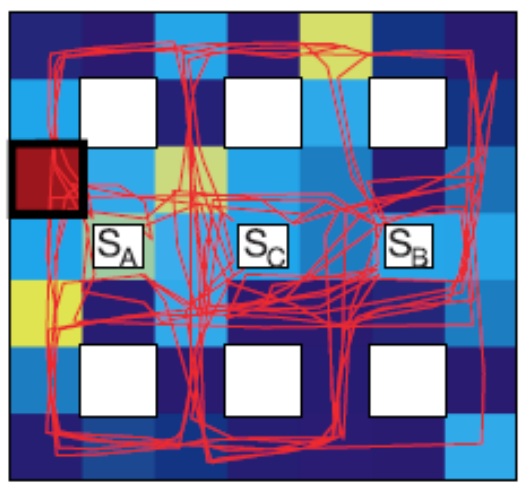

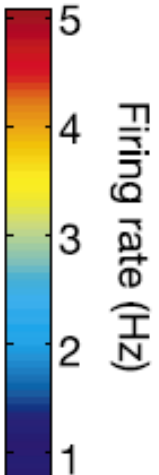

(b)

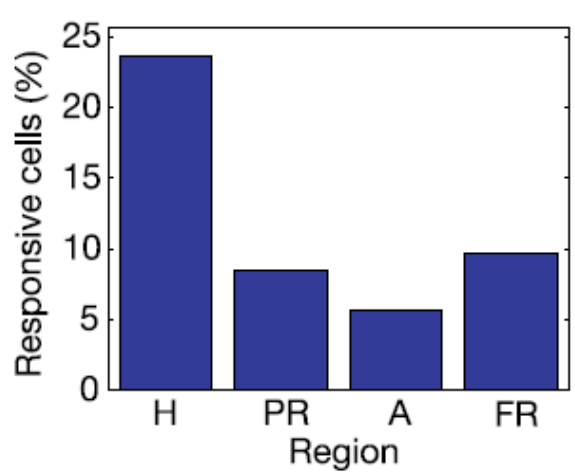

Figure 1.3: Place responsive cells. Letters $S_{A}, S_{B}, S_{C}$ indicate shop locations, white boxes indicate nontarget buildings and the red line indicates the participant's trajectory. (a) Firing rate (in $\mathrm{Hz}$ ) of a right hippocampal cell at various spatial locations. (b) Comparison of place responsive cells in the hippocampus $(\mathrm{H})$, parahippocampal region (PR), amygdala (A) and the frontal lobe (FR). (Courtesy: Ekstrom et al., 2003) 
The indication of the extra-hippocampal medial temporal lobe structures' involvement in spatial memory comes from fMRI studies on patients with lesions. The parahippocampal regions- the perirhinal cortex and parahippocampal cortex belong to the visual processing stream. It has been shown that the perirhinal cortex is crucial for the perception and memory of complex visual objects (Pihlajamaki et al., 2004; Barense, Gaffan and Graham, 2007) whereas the parahippocampal cortex, as suggested by (Ploner et al., 2000) plays a greater role in the processing of visuospatial information. Consistent with these findings was an fMRI study by (Buffalo, Bellgowan and Martin, 2006). In this study, subjects were presented with a series of stimuli similar to those shown in Figure 1.2 (a) and were instructed to memorize the object (Object task) or memorize the location (Spatial task). During the recall phase, subjects were shown three old objects and three new objects (Object task) or objects in three old locations and three new locations (Spatial task). For each stimulus, subjects identified whether the presented object/location is old/new. Notably, the perirhinal cortex exhibited activity during object recognition as well as spatial encoding while the anterior parahippocampal cortex exhibited activity during spatial encoding as seen in Figure $1.2(b)$.

(a)

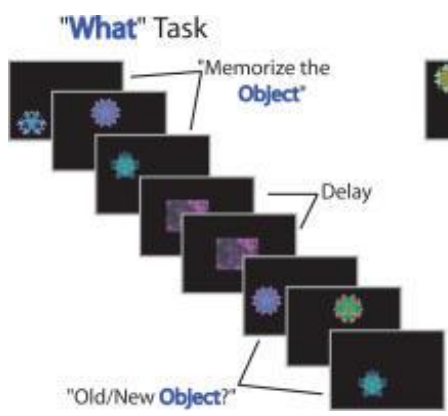

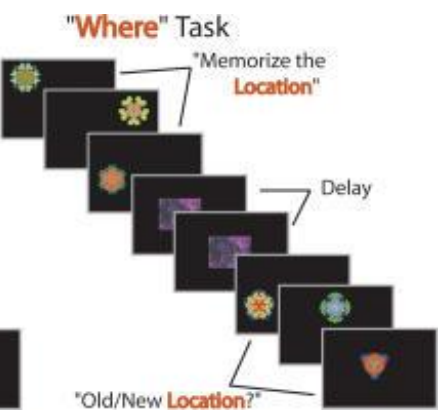

(b)

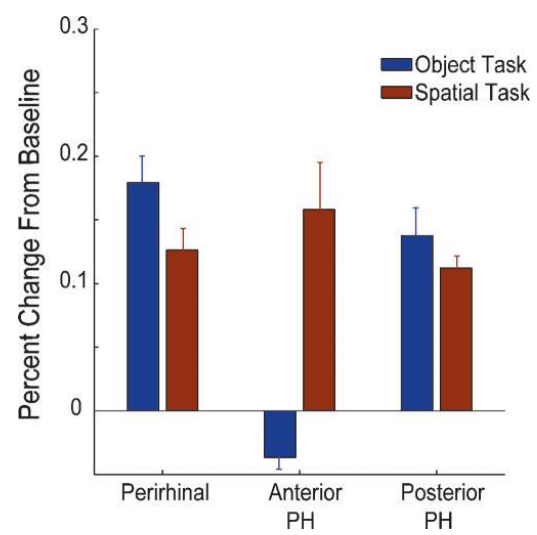

Figure 1.4: (a) Task design for the object task (left) and the spatial task (right). (b) Comparison of the activations in the perirhinal, anterior parahippocampal $(\mathrm{PH})$ cortex and posterior parahippocampal $(\mathrm{PH})$ cortex for the Object task and Spatial Task. (Courtesy: Buffalo, Bellgowan and Martin, 2006) 
Studies on patients who had undergone unilateral lobectomy - a surgical excision of the hippocampus, amygdala and surrounding structures revealed the differences in the memory functions of the left and right temporal lobe structures. In their seminal work, Smith and Milner (1981) provided evidence of spatial memory impairment in patients with right unilateral temporal lobectomy (TL). Smith and Milner tested patients with right and left TL on a task that involved memorizing the names and locations of 16 objects places at various locations on a blank piece of paper. The left TL group showed normal memory for item location while the right TL group showed impaired memory for item location. Subsequent studies confirmed Smith and Milner's findings (Feigenbaum, Polkey and Morris, 1996; Abrahams et al., 1997; Bohbot et al., 1998). Frisk and Milner's experiment on normal subjects and patients with left lobectomy showed distinct capabilities of learning a short paragraph and retaining it in memory. Patients with left TL demonstrated difficulty in learning the story content as well as performed poorly in retention of content after a 20 minute delay (Frisk and Milner, 1990). These results indicate that the left temporal lobe structures play a dominant role in episodic memory whereas the right temporal lobe structures play a dominant role in spatial memory.

Together the findings from previous studies emphasize the involvement of the right medial temporal lobe structures- hippocampus, entorhinal cortex, perirhinal cortex and parahippocampal cortex in spatial memory tasks.

\subsection{Hippocampus and Alzheimer's disease}

Structural MRI scans are most widely used to measure atrophy of the hippocampus for diagnosis of Alzheimer's disease (AD). However, it has been suggested that the onset of pathophysiological process of Alzheimer's disease (AD) occurs years before the clinical diagnosis (Morris, 2005). Based on the scientific evidence available, recently, The National Institute on Aging and the Alzheimer's Association proposed a conceptual framework for diagnosis of pathophysiological process of AD for clinical research studies 
(Sperling et al., 2011). It has been suggested that cognitive studies hold potential for early detection of AD. Therefore, one approach is to use an experimental paradigm that "stresses" the brain or structure that is known to be affected in the preclinical stages of the disease. Since impairment of spatial memory is often one of the first symptoms experienced by patients with dysfunction of the medial temporal lobes (e.g. Kolb and Wishaw, 1996), a spatial learning paradigm can be used to test the activity of the hippocampus. Therefore, determination of alterations in hippocampal function in response to a spatial memory task provides as a sensitive measure of early AD. Evolving modalities such as functional Magnetic Resonance Imaging (fMRI) represents a promising approach for detection of AD associated neurodegeneration.

\subsection{Neuroimaging Techniques}

With the accelerating pace of research in functional neuroimaging there has been an increase in the number of techniques used for the non-invasive assessment of the brain activity. Among these, the most widely used technique is functional Magnetic Resonance Imaging (fMRI). The fMRI technique measures the blood oxygenation level dependent (BOLD) effect. The BOLD effect is an indirect measure of the underlying neural activity evoked due to a stimulus, as shown in Figure 1.3. The neural activity results in local increases in cerebral metabolic rate of oxygen extraction $\left(\mathrm{CMRO}_{2}\right)$, cerebral blood flow $(\mathrm{CBF})$ and cerebral blood volume (CBV). These changes induce a change in the measured MRI signal and is called the BOLD signal. The BOLD signal is explained in greater detail in section 2.4.1. 


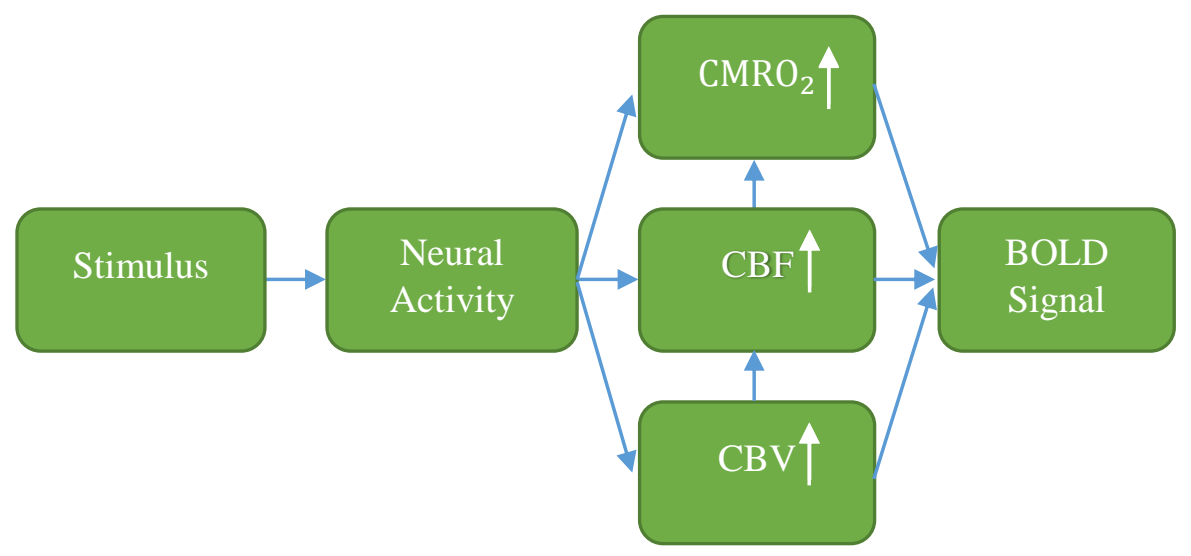

Figure 1.5: The generation of a blood oxygenation level dependent (BOLD) signal in fMRI.

In this study, fMRI is used to acquire volumetric functional scans of the brain. The fMRI technique is favourable over other methods available for functional neuroimaging for a number of reasons. Firstly, fMRI is completely non-invasive and does not involve the injection of an exogenous contrast agent as in the case of Positron Emission Tomography. Secondly, fMRI provides a solution to the spatial - temporal resolution trade-off. fMRI possesses the capability of producing images with temporal resolution higher than Positron Emission Tomography as well as spatial resolution better than Electroencephalography and Magnetoencephalography techniques (Cohen and Bookheimer, 1994). Lastly, fMRI experiments can be performed using a standard clinical MRI scanner.

\subsection{Hypothesis}

During the last three decades, electrophysiological and functional imaging studies on healthy participants as well as patients with lesions and lobectomies, have established the importance of the right medial temporal lobe (MTL) for spatial memory in humans. The hippocampus and its associated structures entorhinal cortex, perirhinal cortex and parahippocampal cortex play a key role in spatial memory, yet the 
underlying physiological hemodynamics remain unknown. Previous studies have shown that the hemodynamic response differs between individuals and probably also between different regions of the brain (Aguirre, Zarahn and D'Esposito, 1998).

In this thesis, we tested the hypothesis that the right hippocampal region together with the surrounding entorhinal, perirhinal and parahippocampal cortices are involved in spatial memory processing and that the hemodynamic responses to a specific spatial task vary among subregions. Furthermore it is also hypothesized that the hemodynamic responses vary among individuals.

In so doing, we evaluated the hemodynamic responses of the seven medial temporal substructures implicated in spatial memory; namely the hippocampal sub-regions (CA1, CA3, Dentate Gyrus, and subiculum), entorhinal cortex, perirhinal cortex and parahippocampal cortex. Since the aim of this study is to investigate the hemodynamic responses to spatial memory, participants are tested on a spatial memory task. While the participants performed the task, they were scanned using the fMRI technique. The acquired scans were pre-processed using an Independent Component Analysis technique to improve the quality of data. The task-related physiological responses of the medial temporal lobe structures were computed from the pre-processed scans using an optimization algorithm proposed in this thesis. 


\section{Theory}

\subsection{Overview}

This chapter summarizes the fundamentals of Magnetic Resonance Imaging (MRI) and functional Magnetic Resonance Imaging (fMRI). The principles of MRI and fMRI are governed by the Nuclear Magnetic Resonance (NMR) phenomenon. This phenomenon was discovered by researchers Edward Purcell at Harvard University and Felix Bloch at Stanford University when samples were placed in a magnetic field. Bloch and Purcell shared the 1952 Nobel Prize for Physics for their joint discovery. In the 1970s, researcher Paul Lauterbur made pioneering contributions for further developing methods for application of the nuclear magnetic resonance phenomenon in diagnostic imaging (Lauterbur, 1973). During the 1990s Ogawa et al., recognized that the differences in magnetic properties of oxygenated haemoglobin and deoxygenated haemoglobin can be exploited to induce changes in the MRI signal. This signal, called the blood oxygenation level dependent (BOLD) signal forms the basis of functional Magnetic Resonance Imaging (fMRI).

\subsection{Nuclear Magnetic Resonance}

The atomic nucleus consists of protons and neutrons. An intrinsic property of protons and neutrons is that they possess an angular momentum or 'spin'. This spin is determined by the number of constituent protons and neutrons in the nucleus. If the number of protons and neutrons in a given nucleus are even, then there exists no net spin. However, if the number of protons and neutrons is odd, the net spin is nonzero and therefore the nucleus also possesses a magnetic moment due to the positive charge of the protons. The hydrogen-1 nucleus consists of odd number of protons $(Z=1)$ and therefore possesses a net spin and 
a magnetic moment. Since the hydrogen molecule is a component of the water molecule which is abundant in the human body, we therefore focus on the hydrogen nucleus.

The NMR phenomenon can be explained using a sample consisting of a large number of hydrogen nuclei. In absence of an applied magnetic field, all the spins in the sample are randomly oriented as shown in Figure. 2.1 (a). These spins cancel each other out and the net spin and net magnetic moment of the sample is zero. However, when the sample is placed in a magnetic field $B_{o}$ applied along the $z$-axis, hydrogen nuclei with spin number $I=1 / 2$ occupy $(2 I+1)$ energy levels. Therefore the hydrogen proton aligns along one of the two orientations: either parallel or anti-parallel to the applied magnetic field $B_{o}$ as shown in Figure. 2.1 (b). The alignment of nuclei parallel to the applied magnetic field $B_{o}$ is the lower energy level and the alignment of nuclei anti-parallel to the applied magnetic field $B_{o}$ is the higher energy level. In the absence of thermal agitation of the nuclei, all spins would attain the lower energy state and the sample would be in its absolute minimum state. However, at physiological temperatures, the thermal agitation leads to a slight excess of spins that align parallel to the applied magnetic field than anti-parallel. Thus, there will be a net magnetic vector $M$ pointing in the direction of the applied magnetic field $B_{o}$ as shown in Figure. 2.1 (c). The applied magnetic field also creates a torque that causes the magnetic moments of the hydrogen nuclei to rotate, or precess, about the $z$-axis. The frequency of this precession is given by the Larmor equation,

$$
v=\gamma B_{o}
$$

where $v$ is the frequency of precession in $\mathrm{MHz}, \gamma$ is the gyromagnetic ratio in $\frac{\mathrm{MHz}}{\text { Tesla }}$ and $B_{o}$ is the magnetic field strength in Tesla. Due to the precession of all the nuclei in phase with each other, the resultant magnetic vector $M$ also rotates about the applied magnetic field $B_{o}$. In order to detect a signal, the spins 


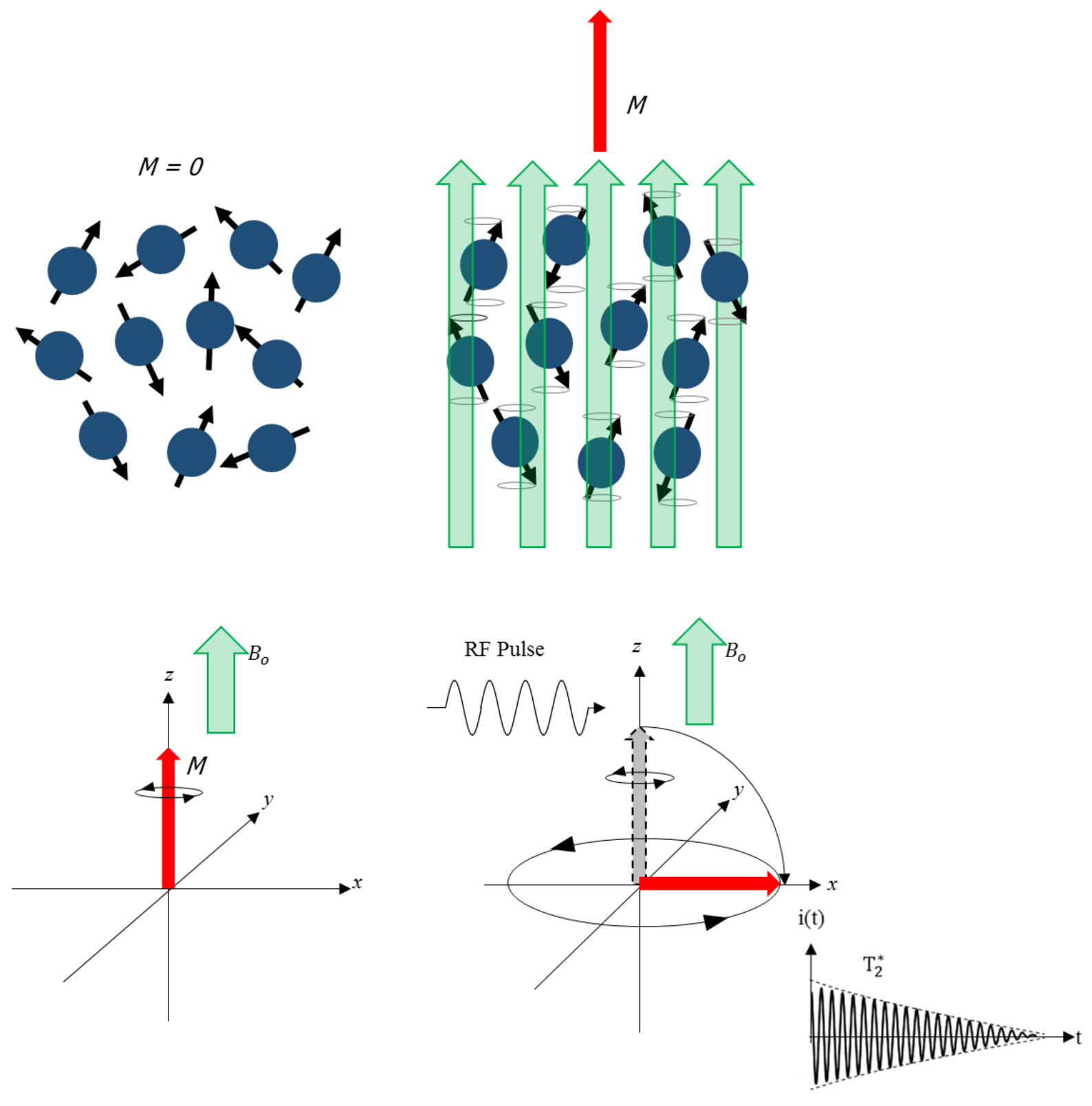

Figure 2.1: Behaviour of a sample when placed in a strong magnetic field. (a) The nuclear magnetic moments are randomly oriented when no external field is applied. (b) When an external magnetic field is applied the moments align either parallel to the field or anti-parallel. (c) The slight preferential alignment parallel to the field gives rise to a net magnetization vector $M$. (d) When an RF pulse is applied, the magnetization vector changes its orientation from the $z$-axis into the $x y$-plane. Due to the non-uniformity of static magnetic field within each voxel and spin-spin interactions, the transverse magnetization vector decays exponentially with time constant $T_{2}^{*}$. 
tip away from the $z$-axis into the transverse $x y$-plane as shown in Figure. 2.1 (d). Because the frequency of this applied magnetic field lies in the radio-frequency range and causes the net magnetization vector to flip by a $90^{\circ}$ angle, it is referred to as a $90^{\circ}$ RF-pulse. The excited spins eventually dissipate their energy and return to the equilibrium state. Therefore the $z$-axis component of the magnetization vector begins to recover. This recovery of the $z$-component is an exponential process and is measured by a time constant $T_{1}$. This is called the $T_{1}$ relaxation process or spin-spin relaxation process.

Right after the $90^{\circ}$ RF-pulse is applied, the net magnetization vector aligned along the $z$-axis is tipped into the $x y$-plane. In a perfect magnet and ideal sample all the spins constituting the net magnetization vector $M$ experience the same magnetic field and continue to precess coherently at the larmor frequency. However, due to random tumbling of neighboring nuclei, the magnetic fields experienced by each nucleus in the sample begins to vary, thereby altering their individual larmor frequencies. The differences in larmor frequencies causes the spins to lose their coherence and the spins begin to de-phase. Therefore, the transverse component of the magnetization vector starts to decay. This decay process is exponential and measured by a time constant $T_{2}$. This is called the $T_{2}$ relaxation process or spin-lattice relaxation process.

Similar to $T_{2}$ decay, $T_{2}^{*}$ decay is also an effect of variations in the magnetic field. However, the processes that drive $T_{2}^{*}$ relaxation are different from those that drive $T_{2}$ relaxation. $T_{2}^{*}$ relaxation is caused due to the varying responses of the different regions of the sample to the applied magnetic field. For example, in the human head, the very steep differences in the magnetic susceptibility of air and tissue at the air/tissue interface causes rapid local $T_{2}^{*}$ relaxation. Similarly varying levels of deoxygenated hemoglobin in the blood vessels affects the $T_{2}^{*}$ in the tissue surrounding the blood vessels. This effect observed in the tissues surrounding the blood vessels due to varying magnetic susceptibility forms the basis of BOLD contrast in fMRI as will be discussed further in this chapter. 


\subsection{Magnetic Resonance Imaging}

The relaxation times $T_{1}$ and $T_{2}$ are tissue type dependent. Typical $T_{1}$ and $T_{2}$ relaxation times for 3T magnetic field for different tissues types in the human brain are given in Table 2.1.

Table 2.1: Typical $T_{1}$ and $T_{2}$ values for grey $\&$ white matter at $3.0 \mathrm{~T}$ magnetic field (Wansapura et al., 1999).

\begin{tabular}{lcc}
\hline & $\boldsymbol{T}_{\mathbf{1}}(\mathbf{m s})$ & $\boldsymbol{T}_{\mathbf{2}}(\mathbf{m s})$ \\
\hline Grey Matter & 1300 & 110 \\
White Matter & 830 & 80 \\
\hline
\end{tabular}

The key step in the advancement of MRI was the realization that a spatially resolved NMR signal can be obtained by using a spatially varying magnetic field. The gradient magnetic field would slightly modify the static magnetic field $B_{o}$. As per the larmor equation, the gradient magnetic field will alter the larmor frequencies of the spins based on their spatial location. Since the larmor frequency for a specific location is known, their spatial locations can be resolved.
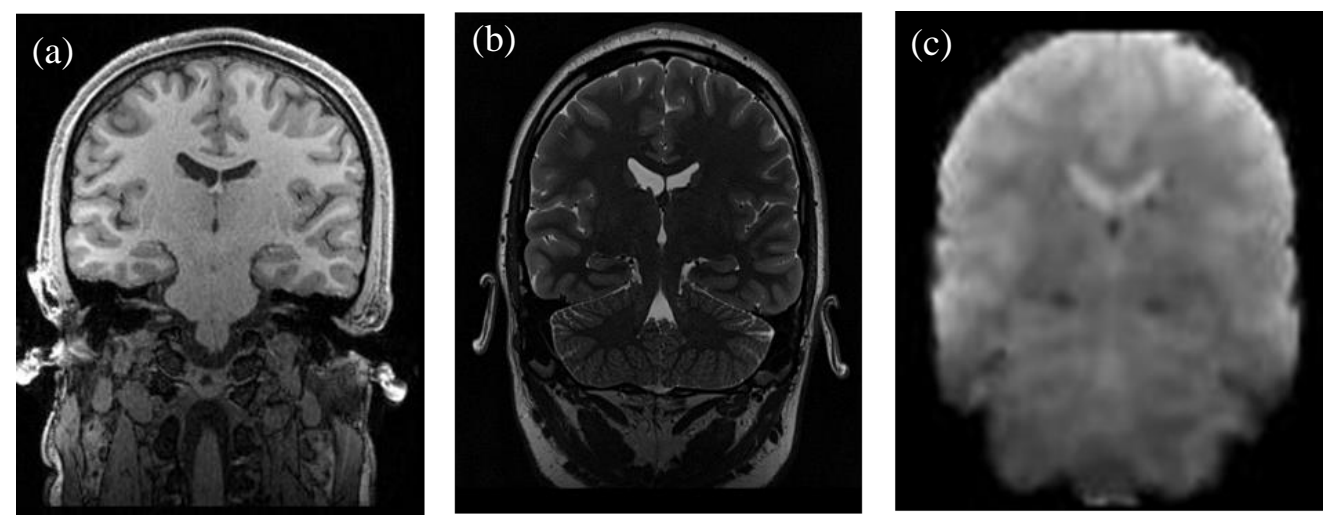
Figure 2.2: Examples of MRI scans. (a) $T_{1}$ - weighted anatomical scan. (b) $T_{2}$ - weighted anatomical scan. (c) $T_{2}^{*}$-weighted functional scan.

\subsection{Functional Magnetic Resonance Imaging}

The aim of fMRI is to assess neuronal activity which is intimately coupled to blood oxygenation level as will be explained in the next section. Since $T_{2}^{*}$ time constant is an indicator of the blood oxygenation levels, $T_{2}^{*}$ time constant is perhaps of more significance to functional Magnetic Resonance Imaging. In-vivo fMRI images with image contrast reflecting the level of oxygenation was first demonstrated by Ogawa et al. in 1990. fMRI is a favourable method for functional imaging for a number of reasons. Firstly, this technique is completely non-invasive and does not involve the injection of an exogenous contrast agent as in the case of Positron Emission Tomography. Secondly, fMRI possesses the capability of producing images with higher spatial resolution compared to Electroencephalography and Positron Emission Tomography. Lastly, fMRI experiments can be performed using a standard clinical MRI scanner. fMRI is used for research studies as well as for pre-surgical investigations. In neuroimaging, fMRI is used to measure brain activity to further our understanding of the brain processes. It is also used to assess brain function prior to surgery (removal of tumor for example) thereby being able to make a well informed decision about a surgery.

The Echo Planar Imaging (EPI) sequence developed by Mansfield (1977) is able to measure the $T_{2}^{*}$ time constant. Because of its high susceptibility to local magnetic field inhomogeneities, EPI is not used for generating anatomical images. However, it is widely used for functional imaging as it is the fastest available MRI sequence.

\subsubsection{BOLD Signal}

The activation of neurons require a supply of energy in the form of adenosine tri-phosphate (ATP) which is generated in the mitochondria within the cells. The formation of ATP via glucose consumption 
requires oxygen. To meet the brain's substantial demand for oxygen, the local cerebral blood flow to that region increases. This oxygen is supplied by the blood attached to a haemoglobin molecule. Haemoglobin $\mathrm{Hb}$ contains iron which possesses magnetic properties. fMRI takes advantage of the differences in the magnetic properties of oxygenated $\mathrm{Hb}$ and deoxygenated $\mathrm{Hb}$. In presence of an attached oxygen molecule to the $\mathrm{Hb}$ molecule, the iron atom is shielded and it behaves as a diamagnet. Therefore the $\mathrm{Hb}$ molecule becomes almost magnetically inactive. This effect causes the spins to de-phase slowly and produces longer $T_{2}^{*}$ times. The absence of oxygen has an opposite effect. Since the iron atom is exposed, the molecule becomes paramagnetic and distorts the magnetic field. This effect causes rapid dephasing of spins, thereby producing very short $T_{2}^{*}$ times. This change in the MR signal which depends on the blood oxygen levels is referred as the blood oxygenation level dependent (BOLD) effect.

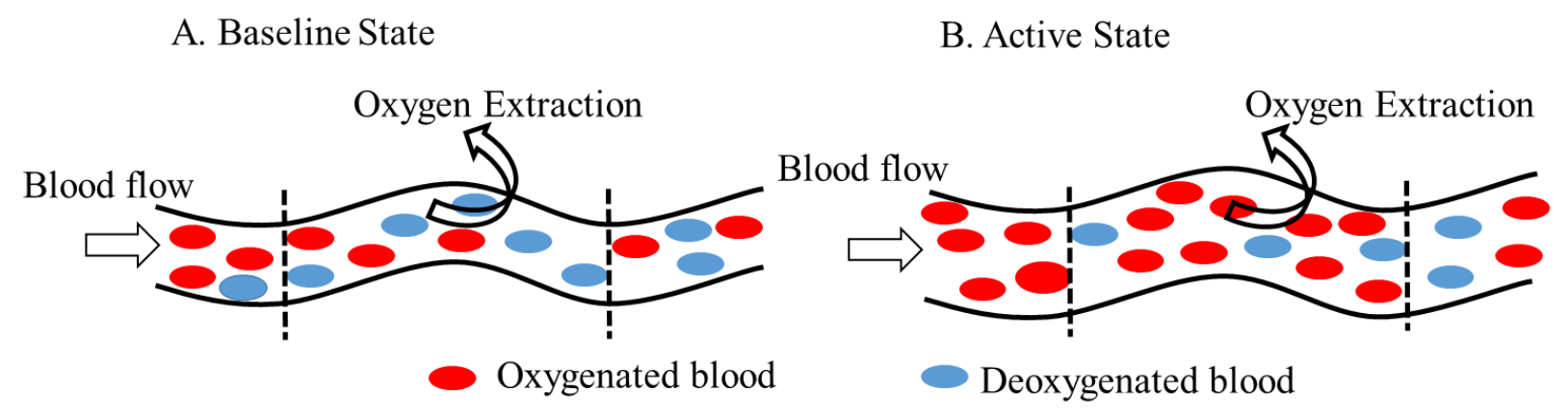

Figure 2.3: (a) In a baseline state the neurons require a certain amount of oxygen. (b) When neuronal activity increases, the demand for also increases thereby increasing the blood flow. This results in a sudden increase in the blood oxygen concentration in an active state compared to the baseline state.

The regional neuronal activity is closely coupled with the blood flow. Logothetis et al., tested this on monkeys by simultaneously measuring the BOLD signal which is an indirect measure of neurones' activity and the EEG which is a direct measure of neurones' electrical activity and concluded that the two are positively correlated. 


\subsubsection{Hemodynamic Response Function}

The intensity variations on a $T_{2}^{*}$ - weighted MR image are observed due to the changes in local blood oxygenation level in the brain induced by an experiment. A model is needed to describe the expected intensity variations due to the stimuli presented in the experimental paradigm. The expected intensity variations or the BOLD signal is thought of as an output of experimental paradigm that passes through two systems (human brain and imaging system) as shown in Figure 2.4.

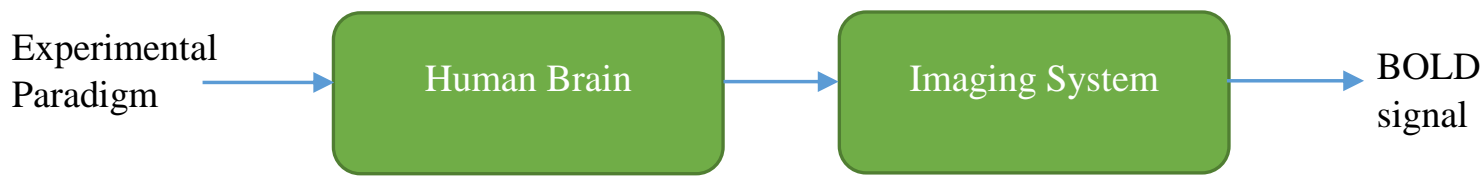

Figure 2.4: The experimental paradigm passes through the brain dynamics and the MRI scanner before being measured as an fMRI signal.

Typically, the BOLD signal has a delayed response to stimuli. Therefore, the relationship between stimuli and the BOLD response is expressed using a convolution. In this model, the human brain and the imaging system are assumed to be linear time invariant (LTI) systems. The linearity assumption holds true in most situations (Boynton et al., 1996). In an LTI system the expected BOLD response is modeled as a convolution of a stimulus function and an impulse response that can be expressed as follows,

$$
X(t)=u(t) \otimes h(\tau)=\int_{0}^{T} u(t-\tau) h(\tau) d \tau
$$

where $\otimes$ denotes the convolution operation, $u(t)$ is the stimulus function and $h(\tau)$ is the impulse response function and it is known as the hemodynamic response function (HRF). A schematic of a typical hemodynamic response is shown in Figure 2.5. The hemodynamic responses are sluggish in nature due to the time delay in supply of blood, initially (1-2s) after the onset of neuronal activity. After the initial delay, oxygenated $\mathrm{Hb}$ is supplied to the activated region via blood. However, the amount of oxygenated $\mathrm{Hb}$ that 
is supplied is more than that extracted from the blood. It is this 'overcompensation' that causes an increase in the oxygenated $\mathrm{Hb}$ level in the blood. This is seen as a positive increase in the MR signal for the next 3$4 \mathrm{~s}$ with a peak at around 5-6s. Once the neuronal activity returns to baseline, the local blood flow to the region decreases. Subsequently, this causes a drop in the MR signal extending below the baseline and is seen as an 'undershoot' in the MR signal (Malonek and Ginvald, 1996). The existence of an 'undershoot' typically depends on the duration of the stimulus (Hirano, Stefanovic and Silva, 2011). For short stimulus durations, only the local vasculature is engaged for supply of oxygenated $\mathrm{Hb}$. The extraction of oxygen from this supply of oxygenated $\mathrm{Hb}$ leads to smaller amounts of deoxygenated $\mathrm{Hb}$ which does not cause an 'undershoot' in the signal. Whereas for long stimulus duration, remote vasculature is also engaged along with the local vasculature causing a large oversupply of oxygenated $\mathrm{Hb}$. The extraction of oxygen from this oversupply of oxygenated $\mathrm{Hb}$ leads to large amounts of deoxygenated $\mathrm{Hb}$ which is represented by a drop in the MR signal as an 'undershoot'. The signal eventually returns to baseline in the next 12-18s.

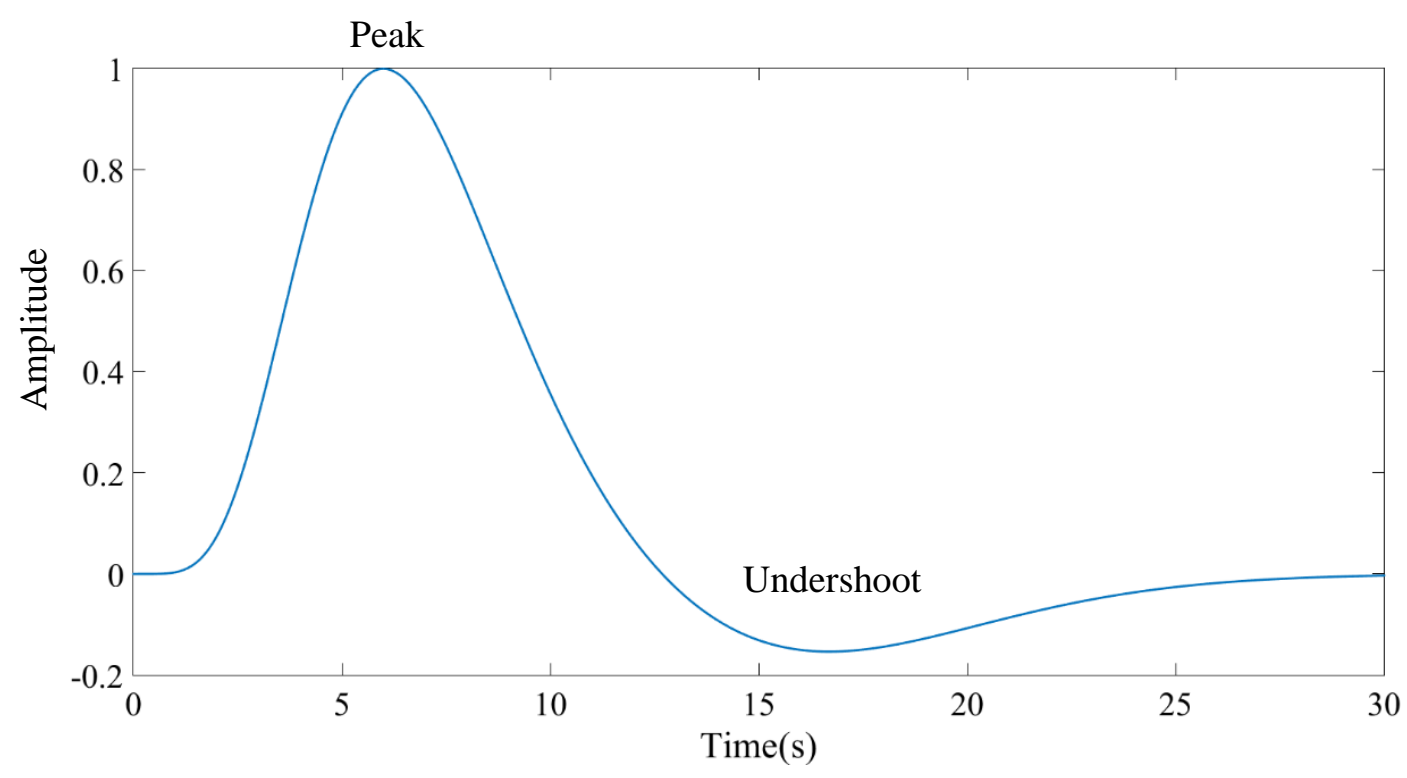

Figure 2.5: Schematic representation of a typical hemodynamic response function. 


\section{Materials \& Methods}

\subsection{Overview}

Seven participants were scanned while they performed a spatial memory task. The task comprised of a series of images of pairs of objects placed at a certain distance from each other. The participants responded as per the instructions that were given to them prior to the scanning session. The scan was performed using a 3.0 Tesla MRI scanner using an echo planar imaging sequence. This chapter provides further details about the participants of the study, the design of the spatial memory experiment and the scanning parameters that were used for acquiring the fMRI images. The computational requirements for the data analysis are also discussed.

\subsection{Participants}

Over 80 young, healthy adults (age group: 21- 30 years) from a cohort of students of University of Colorado, Boulder participated in a screening test. The screening test comprised of a spatial memory task, similar to the task described in section 3.3. Participants were asked to respond accurately and as quickly as possible. Based on each participant's response accuracy (percentage of correct answers) and mean reaction time, the best twenty-four participants were selected for the study (Appendix B). The twenty-four qualified participants were given instructions about the spatial memory task before the scan. After informed consent was obtained, the participants were scanned while they performed a spatial memory task. Participants with poor accuracy and reaction time or severe head movements during scanning were further eliminated from the data analysis. Seven participants' (mean age: $22.86 \pm 2.04$ years) scans were chosen for data analysis. 
All the participants were right- handed individuals with no history of neurological disorders and 20/20 or corrected vision.

Table 3.1: Demographics of the participants

\section{Participant\# Subject ID Gender Age Handedness}

\begin{tabular}{lcccc}
\hline $\mathbf{1}$ & 141 & Female & 23 & Right \\
$\mathbf{2}$ & 131 & Female & 25 & Right \\
$\mathbf{3}$ & 58 & Female & 21 & Right \\
$\mathbf{5}$ & 36 & Male & 21 & Right \\
$\mathbf{6}$ & 5 & Male & 26 & Right \\
$\mathbf{7}$ & 1 & Male & 21 & Right \\
& 0 & Female & 23 & Right \\
\hline
\end{tabular}

\subsection{Spatial Memory Task}

The study design involved viewing of a series of images or 'stimuli' and correctly identifying one of the five presented conditions summarized in Table 3.2. Each stimulus consisted of a pair of objects placed diagonally at a certain distance from each other. When a stimulus with a pair of objects is presented for the first time, it represents a 'new' or 'E0' condition. When a previous pair of objects is repeated, it represents a 'repeat' or 'E1' condition. When a pair of objects is repeated with a small change in the distance between them, it represents a 'lure 1' or 'E2' condition. When a pair of objects is repeated with a medium change in the distance between them, it represents a 'lure 2' or 'E3' condition. When a pair of objects is repeated with a large change in the distance between the objects, it represents a 'lure 3' or 'E4' condition. Since fMRI does not measure absolute neural activity, study designs provide a rest or baseline 
condition to contrast the neuronal activity. Moreover, the brain is constantly engaged in various control functions even during rest. Therefore, a baseline task or a rest period is introduced to be able to quantify the relative changes of brain activity. Interleaved with the E0, E1, E2, E3 and E4 stimuli were the baseline stimuli ' $\mathrm{N}$ ', during which a simple arithmetic problem was presented on pixelated images. The subjects were instructed to add the numbers from the two images in the baseline stimulus and indicate their answer via button press.

Table 3.2: Description of stimuli conditions presented for the spatial memory task.

\begin{tabular}{cc}
\hline Condition & Description \\
\hline E0 & Original/ New \\
\hline E1 & Repeat \\
\hline E2 & Small distance \\
\hline E3 & Medium distance \\
\hline E4 & Large distance \\
\hline $\mathbf{N}$ & Number/Baseline \\
\hline
\end{tabular}

An event related paradigm was used to present the stimuli. Each stimulus was presented for a period of $3 \mathrm{~s}$ during which the participants viewed the stimulus and indicated their responses. 450 stimuli similar to those shown in Figure. 3.1(a) were presented in total. Following each activity and baseline stimulus, a blank screen was presented for a period of 1s for rest as shown in Figure 3.1 (b). Participants viewed stimuli through a mirror attached to the head coil as shown in Figure 3.2 (a). This mirror reflected the projections from E-Prime Version 2.0 (Psychology Software Tools, Inc., Pennsylvania, USA), a software which was used to display the stimuli. The participants responded using an MR-compatible button press. 
(a)

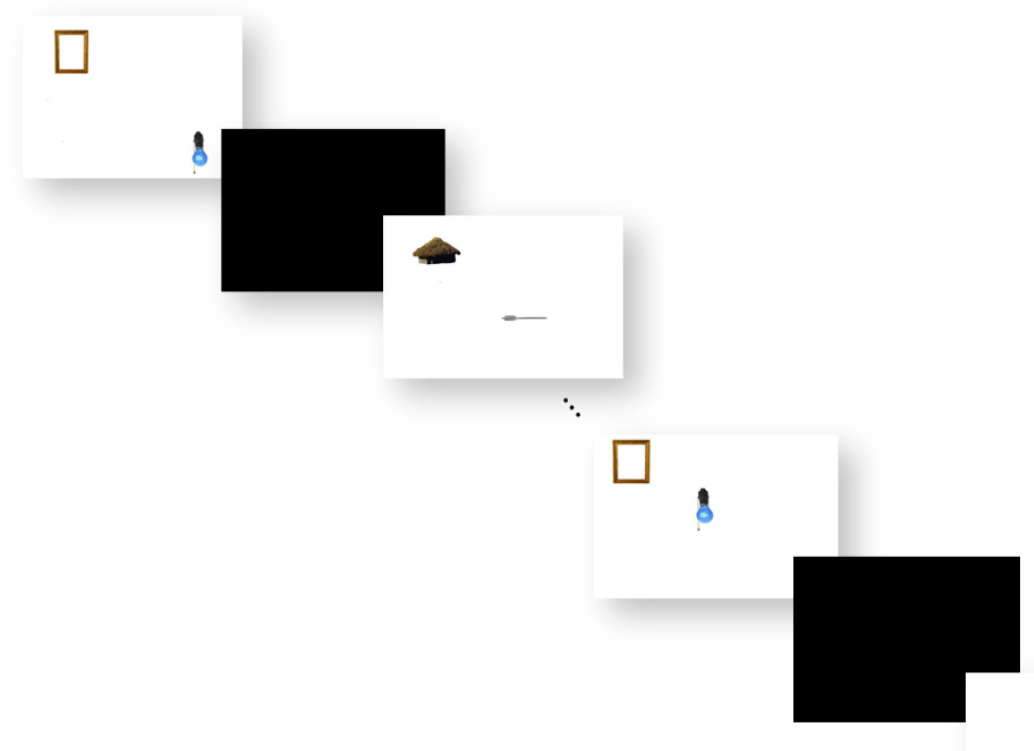

㤦
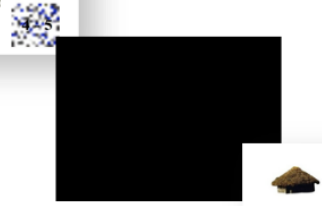

(b)

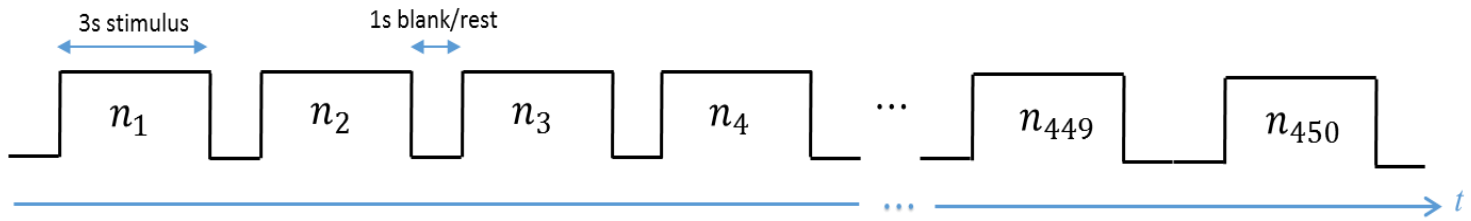

Figure 3.1: Design of the fMRI spatial memory task. (a) Example of stimuli interleaved with baseline task. (b) A total of $n=450$ stimuli were displayed. Each stimulus was displayed for $3 \mathrm{~s}$ followed by a $1 \mathrm{~s}$ blank/rest period. 


\subsection{Image Acquisition}

Before the scanning session, participants were informed about the possible occurrence of symptoms like temporary dizziness or sensory irritations due to the high magnetic field. They were asked for any MR contraindications such as electronic implants (e.g. pacemakers, stimulators, metal splinters in the eye, etc.), electrically conductive implants and prostheses, metallic intra- uterine devices, etc. The participants were requested to remove all electrically conducting objects, e.g. necklaces, rings, braces, hair clips, piercings as well as jewelry. The participants were instructed to remove all clothing and were asked to wear a gown. Each participant was positioned on the MRI table and were asked to lay immobile during the scan because of MR imaging procedures' sensitivity to movement. Two runs of functional scans were collected on a 3.0 Tesla Siemens MAGNETOM TrioTim syngo MR B17 scanner with a 32 channel head coil at the MRI facility of University of Colorado, Boulder.

A set of high-resolution $T_{1}$-weighted whole-brain images were acquired using the following scanning parameters: TE/TR/flip angle $=2.01 \mathrm{~ms} / 2400 \mathrm{~ms} / 8^{\circ}, 224$ slices, voxel size $=0.8 \times 0.8 \times 0.8 \mathrm{~mm}^{3}$ as an anatomical reference prior to the acquisition of functional images. A standard gradient-recalled echoplanar imaging sequence with the following scanning parameters: TR/TE/flip angle $=765 \mathrm{~ms} / 30 \mathrm{~ms} / 44^{\circ}$, voxel size $=1.6 \times 1.6 \times 2.0 \mathrm{~mm}^{3}$, multislice and interleaved mode was used to acquire 2380 volumes of $T_{2}^{*}$-weighted functional images during the spatial memory paradigm as shown in Figure 3.2 (b).

\subsection{Computational Requirements}

A Dell Precision T3610 workstation was used for performing the data analysis. This workstation consisted of Intel@ Xeon@ CPU E5-1620 v2 @ $3.70 \mathrm{GHz}$ processor with 8 cores. The pre-installed 8 GB RAM was upgraded to 48 GB to improve the data analysis performance. The workstation was dual-booted to ensure compatibility of the data analysis software. One hard-drive with Windows 7 Professional (64- 
bit) operating system and an additional hard-drive with Linux (CentOS 6.0) were installed. Software compatible with the Linux operating system like FMRIB's Software Library (FSL), FreeSurfer, dcm2nii and Advanced Normalization Tools (ANTs) were installed on the CentOS. MATLAB was installed on Windows 7 OS.
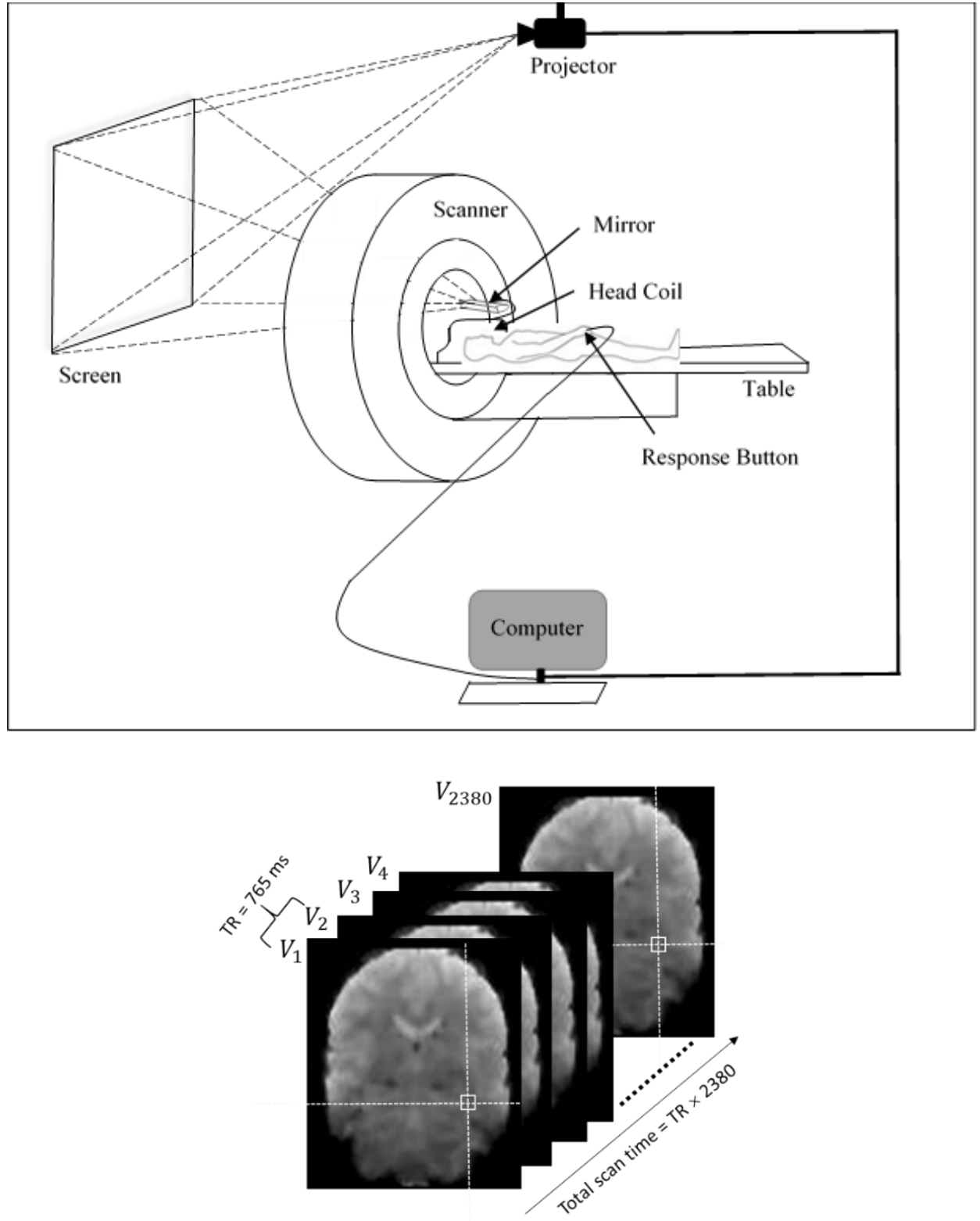

Figure 3.2: (a) Schematic of the fMRI experiment setup. (b) A total of 2380 volumes of $T_{2}^{*}$ weighted scans were acquired every $0.765 \mathrm{~s}$. 


\section{Data Analysis}

\subsection{Overview}

An fMRI examination involves scanning of the brain volume while the subject performs a task. During an fMRI examination, multiple 3-dimensional volumes are acquired over a period of time resulting in a 4-dimensional spatio-temporal data-set. This data-set comprises of some voxels that are 'activated' during the task and their corresponding time course that contains an underlying physiological process of interest i.e. the BOLD response. The activated voxel's time course is also contaminated by fluctuations due to head motion during scanning, cardiac and pulmonary pulses as well as scanner artifacts. The end goal of the fMRI examination is to investigate the BOLD response present in the active voxels. Consequently it is necessary to separate the physiological signal of interest from the non-physiological temporal fluctuations. Hence, fMRI experimental data is usually preprocessed to improve the quality the data.

In general, two categories of data analyses are employed in functional neuroimaging: (i) hypothesisdriven (inferential or model-based), and (ii) data-driven (exploratory or model-free) analysis. The former involves fitting of the observed data to a model for e.g., General Linear Model which is explained later in the chapter. The model uses regressors of interest in a multiple linear regression framework and the estimated regression coefficients are tested against a null hypothesis to generate statistical parametric maps (SPM). The models also make prior assumptions about the spatio-temporal characteristics of the signal. However, the possible presence of unmodeled signals, particularly artefactual activity and the strong prior assumptions which may be invalid, render the analysis sub-optimal. The exploratory data analysis technique is a model free approach that extract features of the underlying signals that are 'interesting' - 
spatio-temporal characteristics of the signal are frequently unknown. Independent Components Analysis (ICA) is an exploratory data analysis approach that provides separation of the BOLD response from noise which makes it an attractive pre-processing tool in fMRI. The statistically independent components generated by ICA can be inspected and be used to separate signal from noise. An automated method for classification of signal and noise components is FMRIB's ICA-based X-noiseifier (Salimi-Khorshidi et al., 2014). For each component FMRIB's ICA-based X-noiseifier generates a large number of distinct spatial and temporal features, each describing a different aspect of the data. The set of features are fed into a oncehand trained multi-level classifier. The noise components can then be regressed out of the original data thus providing an automated 'clean-up' of fMRI data.

\subsection{Independent Components Analysis}

The separation of source signals from a mixture of signals without any prior knowledge of the source signals or the mixing processes is what is known in signal processing as blind source separation (BSS) techniques. The goal of ICA is to solve the BSS problem by expressing a set of observations as linear combination of statistically independent source signals. In classical ICA, a $p$-dimensional vector $X$ consisting of individual observations is assumed to be generated from a linear combination of a set of $n$ statistically independent non-Gaussian source signals $S$ and an unknown mixing function $A$. The $p \times n$ mixing matrix $\mathrm{A}$ is also often assumed to be a square matrix, i.e. $p=n$. The observations can therefore be expressed as,

$$
X=A S
$$

The ICA attempts to find an unmixing matrix $W$, where $W=A^{-1}$ such that each row of matrix $\mathrm{S}$ is mutually independent. However, this technique uses a 'noise- free' model which means that it does not include a model for Gaussian noise sources. This often leads to identification of noise as a 'real effect' and therefore causes 'overfitting' of a noise-free generative model to noisy observations. Another assumption 
that a classical ICA model makes is that the mixing matrix is square. This means that number of source processes is equal to the number of observations which is an invalid assumption for fMRI data. This is because the number of observations in our study is $>100,000$ and we have no reason to believe that there are more than 100,000 source signals present in the data. A solution to these problems is to use a probabilistic ICA approach which assumes that the observed data are confounded by additive Gaussian noise and the mixing matrix is non-square.

\subsubsection{Probabilistic Independent Components Analysis}

Similar to the noise-free ICA model, the probabilistic ICA model characterizes the observed data as a linear combination of source signals and a mixing matrix with an additional assumption that the observations are corrupted by additive Gaussian noise. It further allows for more general non-square mixing. Therefore the model is now given by,

$$
X=A S+E
$$
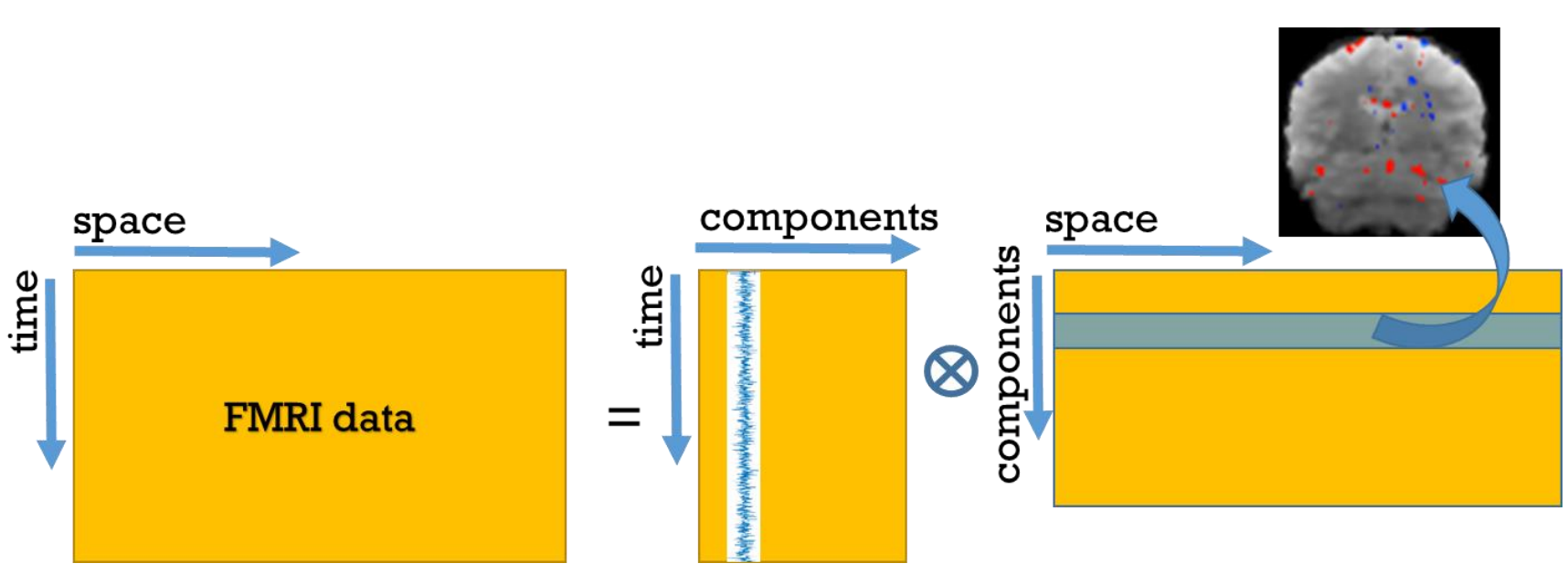

Figure 4.1: Independent Components Analysis- An fMRI spatio-temporal dataset is expressed as a linear combination of independent spatial maps and its associated time courses. 
In analogy to fMRI data, $X$ is a $T \times V$ matrix consisting of $V$ voxels obtained over $T$ time points and $A$ is a $K \times V$ matrix comprising of $\mathrm{K}$ spatial components of independent sources and A is the $T \times K$ matrix of $K$ corresponding time courses as shown in Figure. 4.1. E denotes a p-dimensional vector of Gaussian noise sources.

ICA is believed to work well when a large number of data points are available. Since the fMRI data set acquired in this study consists of a larger number of samples in the spatial domain than the temporal domain, spatial ICA was performed. Moreover, ICA is believed to be more robust in the spatial domain since neural processes in the data may well be more non-Gaussian in space than in time (Smith et al., 2012). Spatial ICA on fMRI data acquired for this study was implemented using FMRIB Software Library's Multivariate Exploratory Linear Optimized Decomposition into Independent Components (MELODIC). Version 3.0 of this software (Beckmann and Smith, 2004) is documented and freely available for download online at (http://fsl.fmrib.ox.ac.uk/fsl/fslwiki/FSL).

The number of independent components to be extracted from the data is a problem of model-order selection. As discussed in the previous section, over-fitting occurs if the model estimators are too flexible allowing it to identify noise sources as neural signals. In contrast, under-fitting occurs if the model estimators are not flexible enough to capture the underlying neural signals in the data. The aim of model order selection is finding an optimal number of independent components such that good estimates of the data are obtained while preventing over-fitting and under-fitting issues. In a nutshell, model order in MELODIC is determined from the covariance matrix of observations using a Bayesian framework. ICA estimation is carried out using the FastICA approach. The extracted spatial maps are converted into ' $Z$ statistic' maps based on the estimated standard error of the residual noise. These maps are assessed for significantly modulated voxels using a Gaussian Mixture Model for the distribution of intensity values. According to Central Limit Theorem, random mixing of signals result in more Gaussian shaped probability density functions (p.d.fs). Conversely if mixing matrix produces non-Gaussian shaped p.d.f, this is unlikely 
to be a random result. The technical details about the procedures in FSL can be found in the previous publications (Jenkinson et al., 2012; Woolrich et al., 2009; Smith et al., 2004).

\subsubsection{Classification of Components}

Once the fMRI data-set is broken down into its independent components, the next step in the process is to identify whether a component is signal or noise. This can be done by visually inspecting each component's thresholded spatial map. One can look at the distribution of activation clusters and the cluster size in the spatial map of a component. A signal component is expected to have a relatively small number of fairly large sized clusters. Whereas, a noise component is expected to have a smaller sized clusters spread throughout the spatial map. Next, the proportion of signal in the high frequency range of a temporal power spectrum is an indicator of whether a component consists of signal or noise. Finally, sudden spikes, sawtooth pattern or drift in the time series is also an indicator of noise. However, manual screening of each component is a time consuming process and also requires a great deal of expertise and is subjective. Instead, a recently introduced automated denoising tool available as an FSL plugin- FMRIB's ICA-based Xnoiseifier (FIX) can be used (Salimi-Khorshidi et al., 2014). FIX attempts to extract more than 180 distinct spatial and temporal features from each component. Spatial features capture various attributes of an IC's spatial map while temporal features capture the dynamics of the time course which help in identifying an IC as "signal" or "noise". Some of the temporal features that are estimated by FIX include skewness of time series, difference between time series mean and its median, time series jump characteristics, ratio of the sum of power above $0.1 \mathrm{~Hz}$ to sum of power below $0.1 \mathrm{~Hz}$, comparing time series with their null model (i.e. convolving white noise with HRF), etc. Some of the spatial features that are estimated by FIX include spatial maps' supra-threshold cluster-size distribution characteristics, balance of negative and positive voxels, edge-mask features, correlation with gray matter, cerebrospinal fluid and white matter masks, etc. 
These features are fed to a hierarchical classifier which makes a decision whether a component is "signal" or "noise". Components that do not fall in the "signal" or "noise" categories are identified as "unknown". Once the signal and noise components are separated, the noise components are subtracted (or regressed) from the data. "Unknown" components are retained in the data.

\subsection{Cortical Reconstruction and Hippocampal Subfield Segmentation}

Cortical reconstruction and volumetric segmentation was performed with the Freesurfer image analysis suite, which is documented and freely available for download online (http://surfer.nmr.mgh.harvard.edu/). The technical details of these procedures are described in prior publications (Dale et al., 1999; Dale and Sereno, 1993; Fischl and Dale, 2000; Fischl, Liu and Dale, 2001; Fischl et al., 2002; Fischl et al., 2004; Fischl et al., 1999; Han et al., 2006; Jovicich et al., 2006; Segonne et al., 2004, Reuter et al. 2010, Reuter et al. 2012). Briefly, this processing includes motion correction and averaging (Reuter et al. 2010) of $T_{1}$ - weighted images, removal of non-brain tissue using a hybrid watershed/surface deformation procedure (Segonne et al., 2004), automated Talairach transformation, segmentation of the subcortical white matter and deep gray matter volumetric structures (including hippocampus, amygdala, caudate, putamen, ventricles) (Fischl et al., 2002; Fischl et al., 2004a) intensity normalization (Sled, Zijdenbos and Evans, 1998) tessellation of the gray matter white matter boundary, automated topology correction (Fischl, Liu and Dale, 2001; Segonne, Pacheco and Fischl, 2007), and surface deformation following intensity gradients to optimally place the gray/white and gray/cerebrospinal fluid borders at the location where the greatest shift in intensity defines the transition to the other tissue class (Dale et al., 1999; Dale and Sereno, 1993; Fischl and Dale, 2000). Once the cortical models are complete, a number of deformation procedures can be performed for further data processing and analysis including surface inflation (Fischl et al., 1999), registration to a spherical atlas which is based on individual cortical folding patterns to match cortical geometry across subjects (Fischl et al., 1999b), parcellation of the cerebral cortex into units with respect to gyral and sulcal structure (Desikan et al., 2006; Fischl et al., 
2004). Freesurfer morphometric procedures have been demonstrated to show good test-retest reliability across scanner manufacturers and across field strengths (Han et al., 2006; Reuter et al., 2012).

Iglesias et al., 2015 manually segmented ultra-high resolution ex-vivo $T_{1}$-weighted MRI scans of fifteen autopsy brain samples. The hippocampal formation was segmented into thirteen component substructures. High precision segmentation was achieved due to the ultra- high resolution of the $T_{1}$ images. These segmentations along with a computational atlas building algorithm based on Bayesian inference methods are used for automatic segmentation of the hippocampal formation in FreeSurfer.

\subsection{Registration}

Registration refers to the spatial alignment of two or more images acquired from different modalities, e.g. registration of a low resolution $T_{2}^{*}$ scan (EPI image) to high resolution $T_{1}$ structural image from the same individual. Registration involves estimating a set of parameters describing a spatial transformation that best matches the images together. This was achieved in two steps as shown in Figure 4.2. First, the anatomical image $T_{1}$ is registered to the mean functional image $T_{2}^{*}$ as shown in Figure 4.2 (a). The $T_{1}$ image is used as a moving image while the mean functional scan is used as the fixed image. The moving image is deformed to match the fixed image. This step produces an output matrix consisting of parameters describing the transformation. The hippocampal segmentation and cortical reconstruction described in the previous section were acquired using a $T_{1}$ image. Therefore, the transformation matrix from the first step can be applied to the segmentation to map the segmentation to functional space as shown in Figure 4.2 (b). An open-source software Advanced Normalization Tools (ANTs) freely available for download online (http://picsl.upenn.edu/software/ants/) was used for registration of segmentation to the functional image (Avants et al., 2008). The registration step enables one to link the anatomical to the functional scans which can then be used for further processing. 
(a)

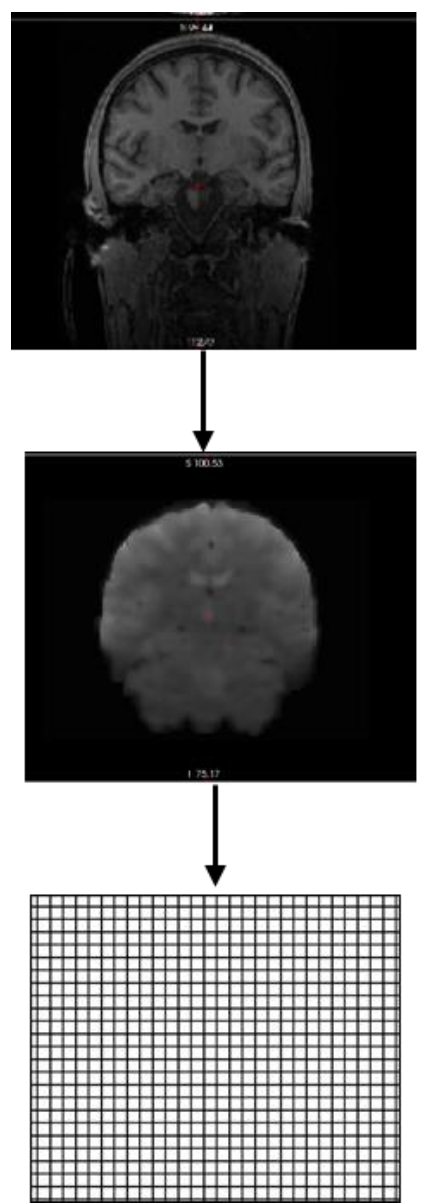

(b)

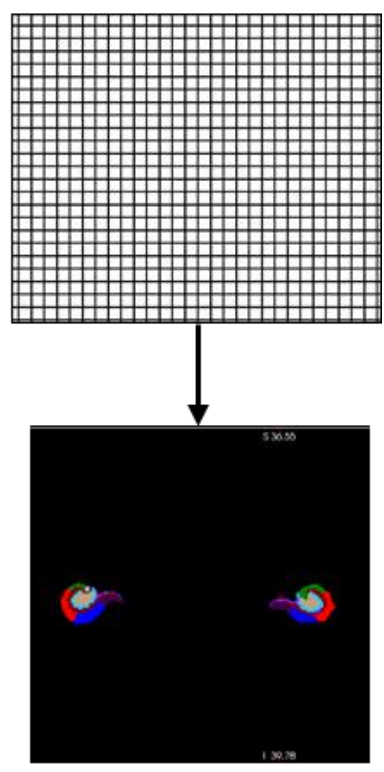

Figure 4.2: Two-step process for registration of the subfields to the functional scan. (a) The anatomical $T_{1}$ - weighted image is registered to the mean functional image $T_{2}^{*}$. This process produces a transformation matrix. (b) The transformation matrix can be applied to the hippocampal subfield segmentation obtained from FreeSurfer since the segmentation is derived from the $T_{1}$-weighted image.

\subsection{The General Linear Model}

This section introduces and provides a brief review of the General Linear Model (GLM) in context with its application to fMRI. The GLM has become the core tool for fMRI data analysis after its introduction to 
neuroimaging by Friston and colleagues (Friston et al., 1994). The most common approach is a mass univariate analysis which constructs a model for each voxel independently.

GLM treats the observed fMRI time course as a linear combination of predictor variables. Linear refers to the weighted sum of several predictor variables. The GLM is defined by the equation:

$$
Y=X \beta+\varepsilon \quad \varepsilon \sim N(0, V)
$$

where $Y$ is the observed fMRI time course of a voxel, matrix $X$ comprises of predictor time courses or the BOLD response as column vectors and is known as the design matrix and $\beta$ are associated coefficients of $X$, quantifying its potential contribution in explaining the voxel time course $y . \varepsilon$ represents the residual errors, prediction errors or noise which quantify the deviation of the measured voxel time course from the predicted time course.

In the matrix form the General Linear Model can be expressed as,

$$
\left[\begin{array}{c}
y_{1} \\
y_{2} \\
y_{3} \\
\vdots \\
y_{n}
\end{array}\right]=\left[\begin{array}{cccc}
1 & X_{11} & X_{12} & \cdots X_{1 p} \\
1 & X_{21} & X_{22} & \cdots X_{2 p} \\
1 & X_{31} & X_{32} & \cdots X_{3 p} \\
\vdots & \vdots & \vdots & \vdots \\
1 & X_{n 1} & X_{n 2} & \cdots X_{n p}
\end{array}\right] \times\left[\begin{array}{c}
\beta_{0} \\
\beta_{1} \\
\beta_{2} \\
\vdots \\
\beta_{p}
\end{array}\right]+\left[\begin{array}{c}
\varepsilon_{1} \\
\varepsilon_{2} \\
\varepsilon_{3} \\
\vdots \\
\varepsilon_{n}
\end{array}\right]
$$

The design matrix $\mathrm{X}$ comprises of predictor time courses or the BOLD response as column vectors. The BOLD signal is modeled as a convolution of the reference function with the hemodynamic response function (HRF) as shown in Figure 4.3. The reference function, also called as regressor, corresponds to the ideal time course of expected BOLD response. It is represented as a box-car function with active conditions defined by values 1 ('on state') and 0 ('off state') at all other time points for different conditions of the experimental paradigm. The regressors of interest for this study are summarised in the Table 4.1. The Difference of Gamma functions HRF model is used similar to (Glover, 1999). 
(a)

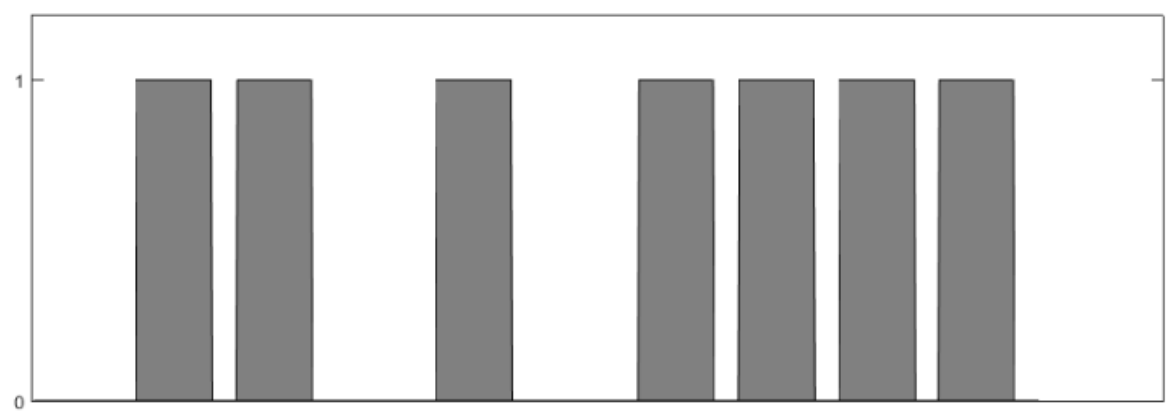

(b)

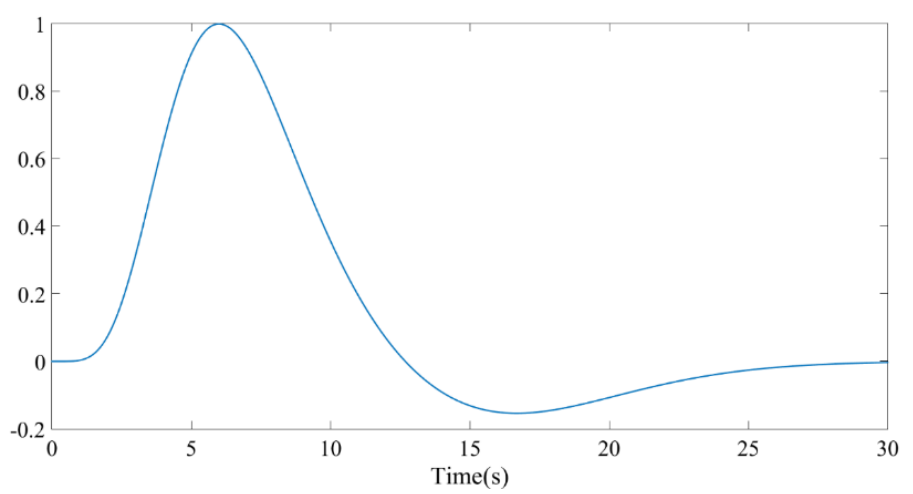

(c)

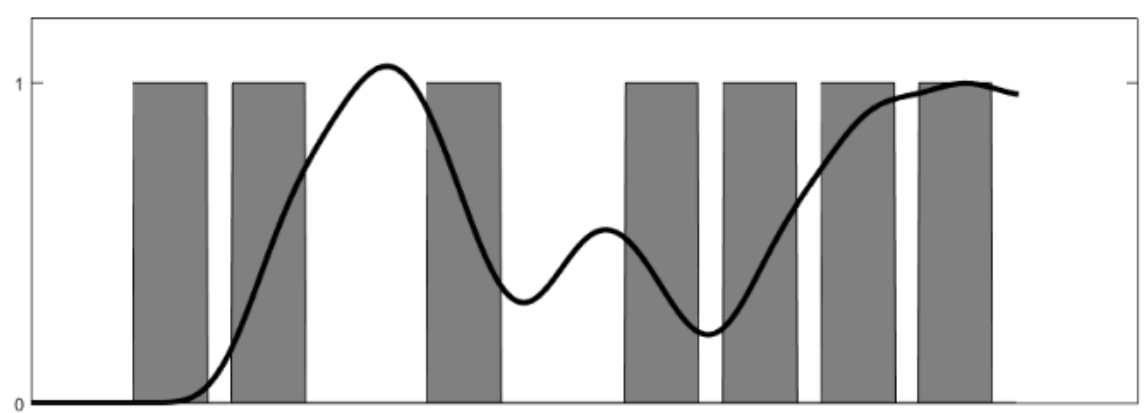

Figure 4.3: Modelling the BOLD response. (a) The reference function is specified as a box-car function with ' 1 ' indicating the 'ON' condition and ' 0 ' indicating the 'OFF' condition. This function is convolved with (b) the hemodynamic response function to produce (c) the expected BOLD signal.

Table 4.1: The regressors of interest.

Regressor Description

E0|E0
Participant correctly responded 'E0' when the E0 condition was presented. 


\begin{tabular}{|c|c|}
\hline$\sim$ E0|E0 & $\begin{array}{l}\text { Participant did not respond 'E0' when the } \\
\text { E0 condition was presented. }\end{array}$ \\
\hline E1|E1 & $\begin{array}{l}\text { Participant correctly responded 'E1' } \\
\text { when the E1 condition was presented. }\end{array}$ \\
\hline$\sim$ E1|E1 & $\begin{array}{l}\text { Participant did not respond 'E1' when the } \\
\text { E1 condition was presented. }\end{array}$ \\
\hline E2 E3 E4|E2 & $\begin{array}{l}\text { Participant responded 'E2', 'E3' or 'E4' } \\
\text { when the E2 condition was presented. }\end{array}$ \\
\hline$\sim \mathrm{E} 2 \mathrm{E3}$ E4|E2 & $\begin{array}{l}\text { Participant did not respond 'E2', 'E3' or } \\
\text { 'E4' when the E2 condition was } \\
\text { presented. }\end{array}$ \\
\hline E2 E3 E4|E3 & $\begin{array}{l}\text { Participant responded 'E2', 'E3' or 'E4' } \\
\text { when the E3 condition was presented. }\end{array}$ \\
\hline$\sim \mathrm{E} 2 \mathrm{E3}$ E4|E3 & $\begin{array}{l}\text { Participant did not respond 'E2', 'E3' or } \\
\text { 'E4' when the E3 condition was } \\
\text { presented. }\end{array}$ \\
\hline E2 E3 E4|E4 & $\begin{array}{l}\text { Participant responded 'E2', 'E3' or 'E4' } \\
\text { when the E4 condition was presented. }\end{array}$ \\
\hline E2 E3 E4|E4 & $\begin{array}{l}\text { Participant did not respond 'E2', 'E3' or } \\
\text { 'E4' when the E4 condition was } \\
\text { presented. }\end{array}$ \\
\hline N duration & $\begin{array}{l}\text { The duration for which the baseline } \\
\text { stimulus was presented }(3 \mathrm{~s}) \text {. }\end{array}$ \\
\hline Begin duration & Ten seconds countdown before the task. \\
\hline Blank Duration & The 1s rest/blank period after a stimulus. \\
\hline $\begin{array}{l}\text { Blank Duration after } \\
\text { number stimulus }\end{array}$ & $\begin{array}{l}\text { The } 1 \mathrm{~s} \text { rest/blank period after a baseline } \\
\text { stimulus. }\end{array}$ \\
\hline End & $\begin{array}{l}\text { The duration after the end of the task } \\
\text { while scanner is still on. }(\sim 10.7 \mathrm{~s})\end{array}$ \\
\hline
\end{tabular}

Given the observed data $\mathrm{y}$ and the design matrix $\mathrm{X}$, the goal of the GLM is to find a set of $\beta$ such that the sum of square error is minimized. The predictor time courses for the model are given by:

$$
\hat{y}=X \beta
$$


Therefore rearranging the equations, we get:

$$
\varepsilon=y-X \beta=y-\hat{y}
$$

The sum of squared error becomes:

$$
\varepsilon \varepsilon^{\prime}=\varepsilon^{2}=(y-X \beta)(y-X \beta)^{\prime}
$$

The optimal $\beta$ estimates are the least square estimates determined by,

$$
\beta=\left(X^{\prime} X\right)^{-1} X^{\prime} y
$$

The term $X^{\prime} X$ results in a square matrix with number of rows and columns corresponding to the number of predictors. Each value contained in the $X^{\prime} X$ matrix is a scalar product of two predictor vectors. The scalar product is obtained by summing all products of corresponding entries of two vectors corresponding to the calculation of covariance. This $X^{\prime} X$ matrix, thus, corresponds to the predictor variance-covariance matrix. Each term in the $X^{\prime} y$ matrix is a scalar product (covariance) of a predictor time course with the observed voxel time course.

\subsection{Algorithm for deconvolution of time series}

Deconvolution of the time series was performed using a cross-validation approach (e.g. Mahmoudi et al., 2012). Cross-validation was carried out in two steps: in the estimation step, using one half of the time series a set of parameters of the hemodynamic response function (HRF) were estimated. In the validation step, using the other half of the time series, the best set of parameters is determined by choosing the set of parameters from the previous step that minimize the optimization criterion. These steps were repeated by interchanging the part of the time series used for estimation and validation. The average of parameters from the two processes was considered to be the optimal set of parameters for a given voxel. 
In the following sections, details of the algorithm are specified. The algorithm is summarized in a flowchart in Figure 4.5.

\subsubsection{Selection of voxels}

The individual segmentation produced by FreeSurfer are registered to the mean functional scan of a run for a given participant. The segmentation can now be overlaid on the corresponding pre-processed 4dimensional functional run to select a region of interest (ROI). The co-ordinates of the voxels of the mean functional scan overlapping with the segmentation are recorded. The time series for individual voxels for a given run are extracted by recording the voxel's values at each of these co-ordinates from all the functional scans. The number of voxels recorded within each sub-region averaged across all participants are summarised in Table 4.2.

Table 4.2: Number of voxels recorded within each sub-region averaged across all the participants and their corresponding standard deviations.

\begin{tabular}{ccc}
\hline Sub-region & Left & Right \\
\hline CA1 & $169 \pm 15$ & $184 \pm 23$ \\
\hline CA3 & $66 \pm 7$ & $70 \pm 12$ \\
\hline Subiculum & $94 \pm 19$ & $119 \pm 21$ \\
\hline Dentate Gyrus & $103 \pm 5$ & $108 \pm 11$ \\
\hline Entorhinal Cortex & $240 \pm 28$ & $288 \pm 34$ \\
\hline Perirhinal Cortex & $210 \pm 22$ & $232 \pm 27$ \\
\hline Parahippocampal Cortex & $497 \pm 35$ & $568 \pm 41$ \\
\hline
\end{tabular}




\subsubsection{Hemodynamic response function model}

Various forms of the hemodynamic response function (HRF) such as Poisson model (Friston et al., 1994), Gamma model (Boynton et al., 1996), Gaussian model (Rajapakse et al., 1998) and Difference of Gamma model (Friston et al. 1998) have been introduced. In this study, the widely used Difference of Gamma HRF model is used which is able to model the delay and undershoot observed in the hemodynamic response. The "standard" Difference of Gamma HRF which can be expressed as,

$$
\begin{gathered}
h r f=\left(\frac{t}{6}\right)^{6} e^{-(t-6)}-\frac{1}{6}\left(\frac{t}{16}\right)^{16} e^{-(t-16)} \\
h r f=\left(\left(\frac{t}{6}\right)^{6} e^{6}-\frac{1}{6}\left(\frac{t}{16}\right)^{16} e^{16}\right) e^{-t} \\
h r f=\left(t^{6}\left(\frac{1}{6}\right)^{6} e^{6}-\frac{1}{6} t^{16}\left(\frac{1}{16}\right)^{16} e^{16}\right) e^{-t}
\end{gathered}
$$

The general HRF is modeled by making 6 and 16 arbitrary constants.

Substituting $a_{1}=\left(\frac{1}{6}\right)^{6} e^{6}, a_{2}=6, a_{3}=\left(\frac{1}{16}\right)^{16} e^{16}, a_{4}=16$

$$
h r f=\left(a_{1} t^{a_{2}}-a_{3} t^{a_{4}}\right) e^{-t}
$$

Therefore, a seven parameter HRF can be reduced to a four parameter HRF for simplifying the estimation process. We can normalize the HRF using $a_{1}=1$. The remaining three parameters of the HRF can be abbreviated by,

$$
x=\left\{a_{2}, a_{3}, a_{4}\right\}
$$




\subsubsection{Estimation of the HRF parameters}

The observed time course of a voxel is split into two halves. One half of the time series is used for estimation, other half is used for validation step. The design matrix $X$ is first set up with the columns as regressors. The regressors of interest are summarised in the Table 4.1. E0 represents a new stimulus, E1 represents a repeated stimulus with same distance between objects, E2 represents a repeated stimulus with small distance change between objects, E3 represents a repeated stimulus with medium distance change between objects, and E4 represents a repeated stimulus with a large change in distance between objects. These regressors are convolved with the HRF to create a design matrix X. Since the TR at which original data is acquired is $0.765 \mathrm{~s}$, the design matrix is also sampled every $0.765 \mathrm{~s}$. The $\beta$ or the linear least squares solution $X^{+} y$.

A set of optimal parameters that minimizes the optimization criterion are determined for different values of average curvature $(\mu)$ of HRF. The objective function or optimization criterion is then stated as:

$$
\left\{x_{\lambda, \mu}\right\}=\arg \min _{x}\left(\overline{\eta(x)}+\lambda\left|\int_{0}^{30 s}\right| \frac{\partial^{2} h r f(x, t)}{\partial t^{2}}|d t-\mu|\right) \text { for fixed } \lambda, \mu
$$

where $\overline{\eta(x)}$ is the mean squared error computed as the variance of (y-X $\beta$ ). The penalty term $\lambda$ is L1 norm-type that penalizes the curvature of the HRF. According to the concepts of numerical optimization, the L1 norm-type penalties are exact and it is sufficient to find one appropriate value of $\lambda$ that is large enough where solution of equality constraint is satisfied for a given average curvature $\mu$ (Nocedal and Wright, 2006). In our case, $\lambda=1$ for all $\mu \in[1,30]$. The term $\int_{0}^{30 s}\left|\frac{d^{2} h r f(x, t)}{d t^{2}}\right| d t$ represents the curvature of the HRF averaged over the typical time duration of an HRF i.e. 0-30s. The optimization problem is solved using the common Nelder-Mead algorithm, a derivative-free optimization method available in MATLAB (The MathWorks, Inc.). 


\subsubsection{Validation of parameters}

Once a set of optimal parameters for each curvature of HRF $(\mu)$ are determined, the other half of the time course is chosen. The HRF is set up using parameters obtained from the previous step. The optimized parameters are determined by,

$$
x_{\lambda, \mu}^{*}=\arg \min _{\{\bar{x}, \mu\}} \overline{(\eta(x))}
$$

\subsubsection{Determining the best parameters}

The estimation and validation steps are repeated by interchanging the part of time course used for each step. Another optimal set of parameters are generated using this approach. The best parameters are determined by calculating the mean of the parameters determined from the two approaches. The mean of the parameters is considered to be the optimal set of parameters for the given voxel. 
For each voxel, split the time series into two. Choose Datal = first half of the time series. Data2 $=$ second half of the time series.

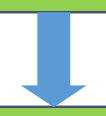

Set up the design matrix $X=\left[X_{1} \ldots X_{15}\right]$ and sample at TR $=0.765 \mathrm{~s}$.

Calculate residual squared error $\eta=\varepsilon \varepsilon^{\prime}=\varepsilon^{2}=(y-X \beta)(y-X \beta)^{\prime}$

Where $\mathrm{y}$ is one half of the voxel time series and $b=X^{+} y$ is the linear least squares solution for

GLM. Determine optimal set of parameters for each mu that minimizes the optimization criterion:

$$
\left\{x_{\lambda, \mu}\right\}=\arg \min _{x}\left(\overline{\eta(x)}+\lambda\left|\int_{0}^{30 s}\right| \frac{d^{2} h r f(x, t)}{d t^{2}}|d t-\mu|\right) \text { for fixed } \lambda, \mu
$$

Using each set of parameters obtained from the previous step, the value of the optimization criterion is determined for the other half of the time series. The set that gives the minimum value of optimization criterion is the optimal set for a specific time series.

$$
x_{\lambda, \mu}^{*}=\arg \min _{\left\{x_{\lambda, \mu}\right\}} \overline{(\eta(x))}
$$

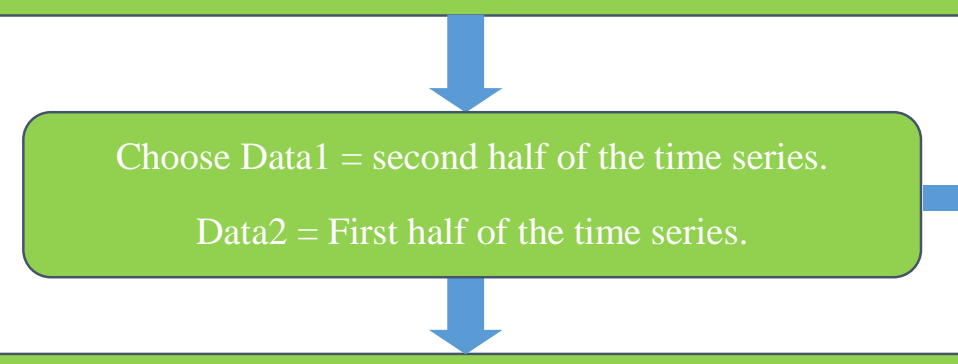

Average of the parameters from the two iterations is used to determine the HRF for the given time series.

Figure 4.4: Flowchart of the algorithm. 


\section{Results}

\subsection{Overview}

Independent components analysis (ICA) was used for obtaining independent spatial maps and associated time courses of the fMRI data set for each participant. Pre-processing was performed using FIX to remove the noise components. The pre-processed data-set along with the hippocampal subfields and cortical labels are used to obtain the hemodynamic response functions of the different regions.

In this chapter, the independent components generated by ICA are presented. A visual inspection of the components is carried out to illustrate how 'signal' and 'noise' components are identified by FIX. Next, the segmentation of the hippocampal formation and the cortical reconstruction are shown. The hippocampal subfields obtained after the segmentation process are registered to the functional MRI scan. Lastly, the hemodynamic response functions of the hippocampal and parahippocampal regions are presented.

\subsection{Independent Components Analysis Results}

The ICA process for each fMRI data-set using FSL's MELODIC takes about 72- 96 hours to complete. Since an 8 core workstation was used for this study, it was possible to process 3-4 fMRI datasets simultaneously. Example of two independent spatial maps as estimated by ICA are shown in Figure 5.1. The corresponding z-scores for both spatial maps are presented indicating the regions of activation (blue), inactivation (yellow) and intermediate activation/inactivation. As can be seen from Figure 5.1(a), the activation clusters in component 1 are fairly small in size and uniformly spread throughout the spatial map. These activation are not within the white matter- grey matter boundaries indicating that this 
component is a noise component. This can be further confirmed by inspecting the power spectrum and the time course of the signal. As seen in the power spectrum Figure 5.2(a), a large proportion of the signal belongs to the high frequency range. The time course of the signal demonstrated a sawtooth like pattern indicating the signal is due to noise or artefact.

As seen in Figure 5.1(b), the activation clusters are large in size and localised within the grey matter or white matter boundaries. A major proportion of the power spectrum of component in Figure 5.2(b) lies in the low frequency range and the time course does not look artefact related. Therefore this component can be identified as a signal component. Since manual classification of components is subjective and rather time consuming, FIX is used for classification of the components instead. It takes about 6-8 hours for the FIX process to complete.

(a)

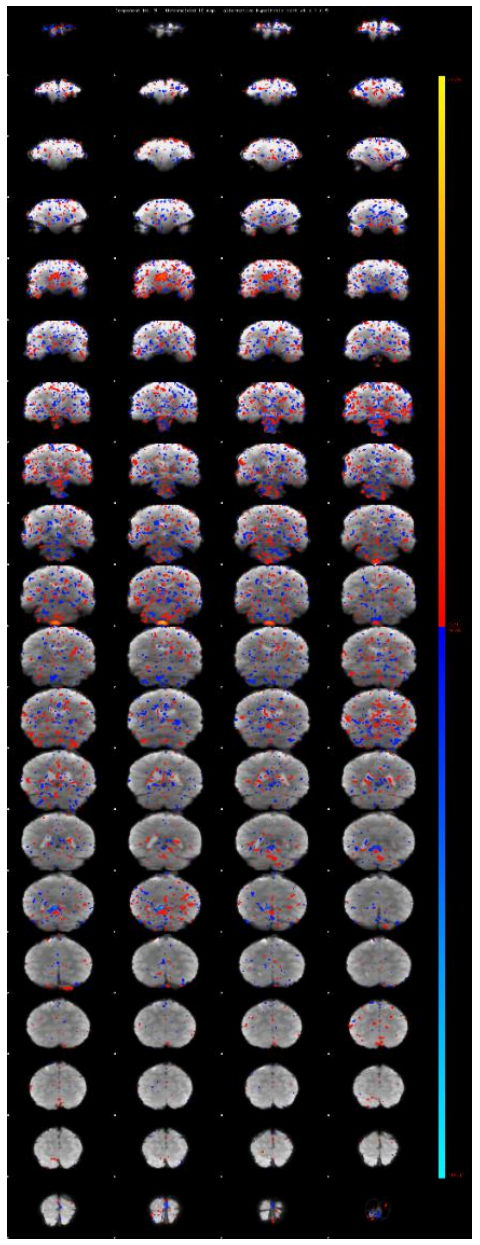

(b)

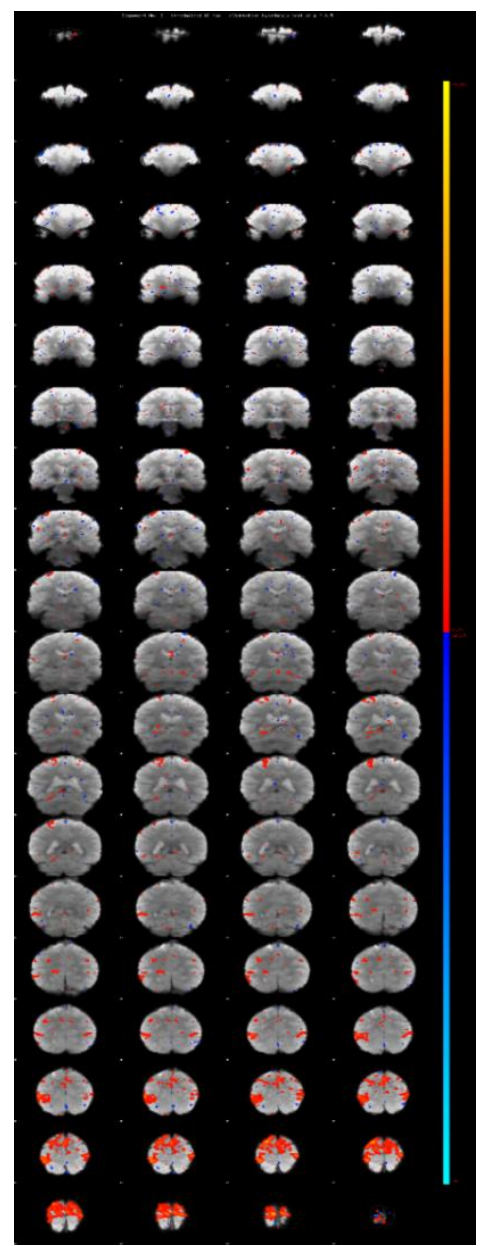

Figure 5.1: Example of spatial component maps generated by ICA. (a) Component 1: Noise (b) Component 2: Signal. 
(a)

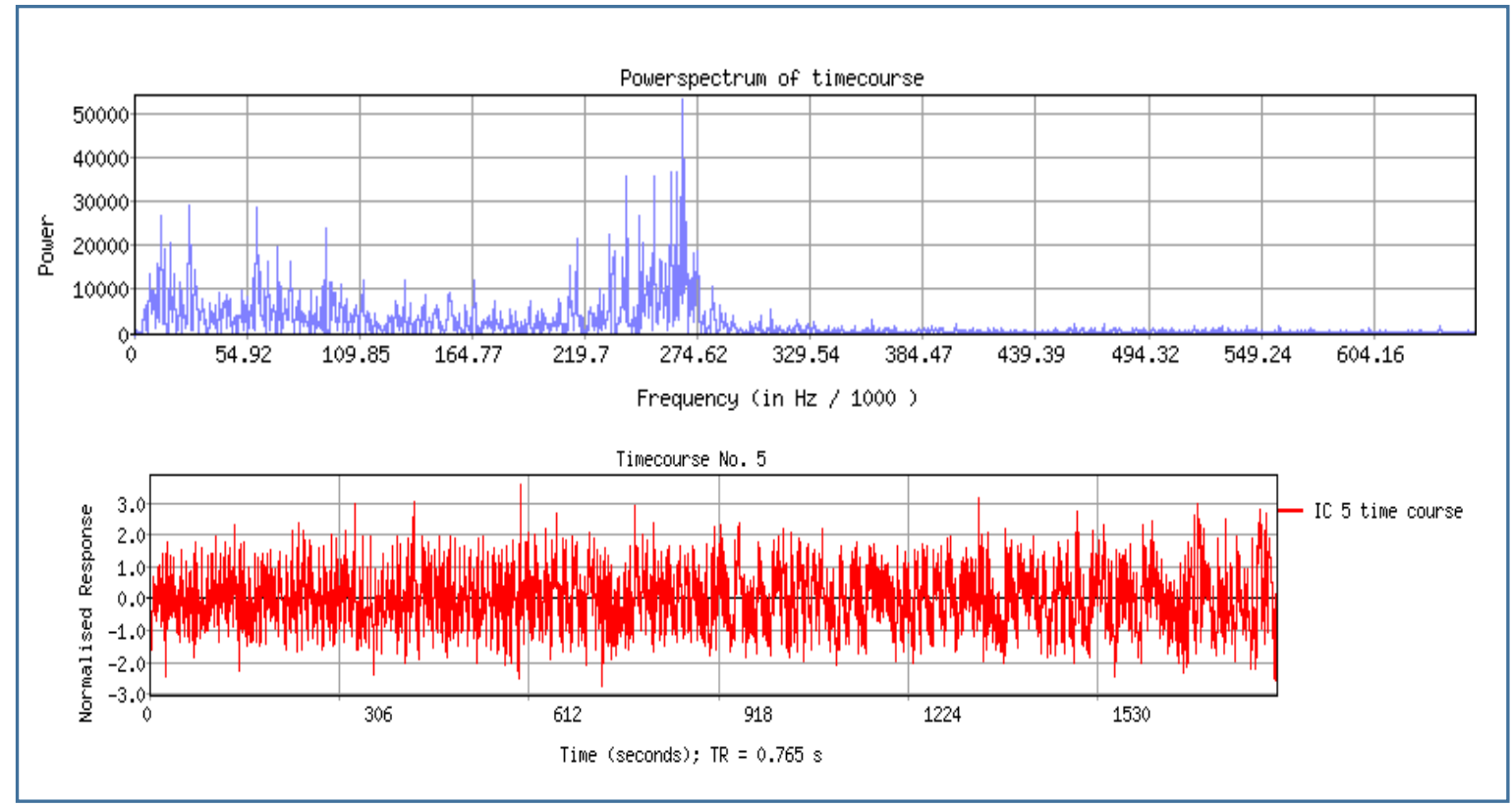

(b)

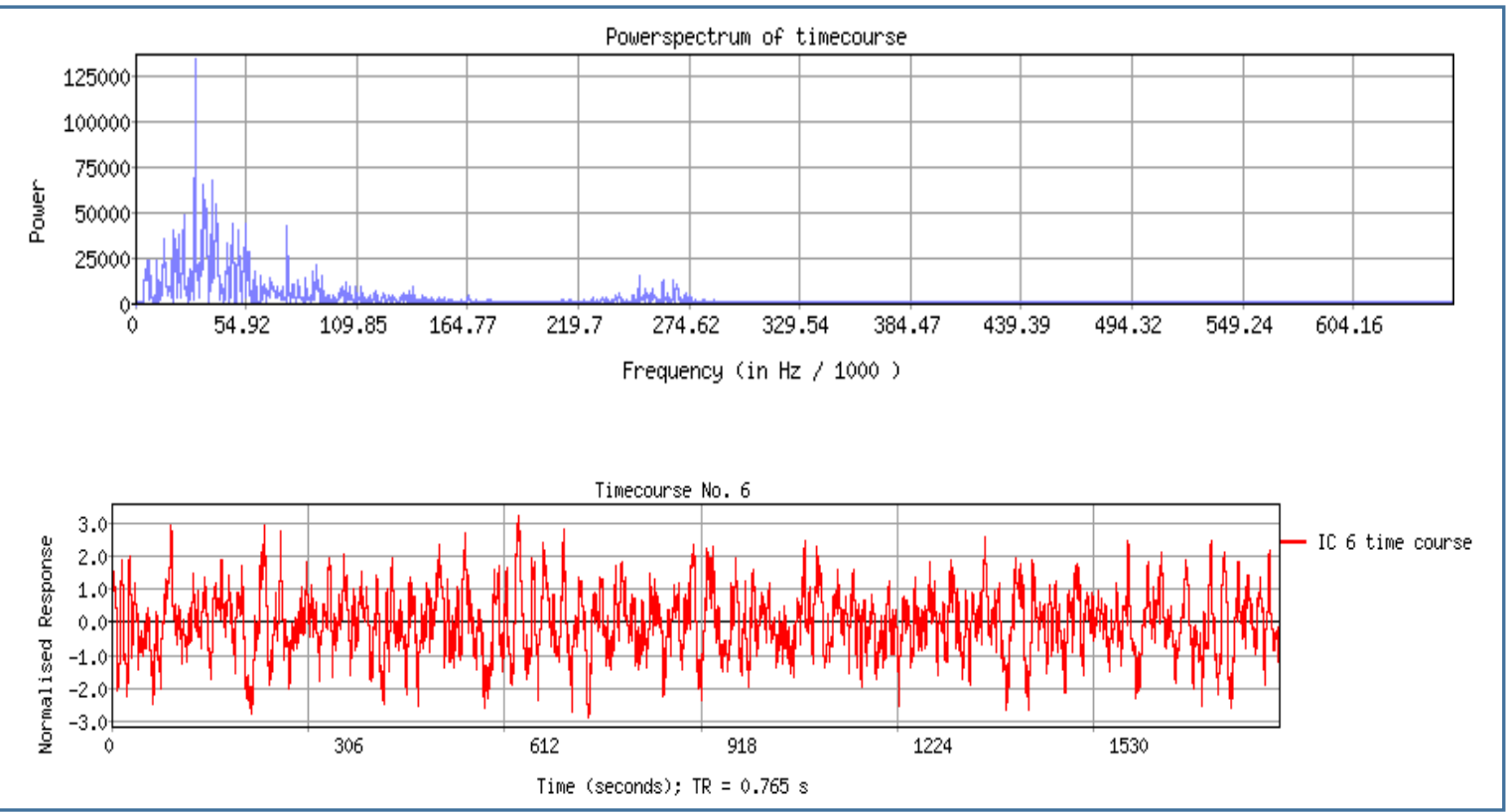

Figure 5.2: The power spectrum and time course associated with the spatial maps. (a) Component 1: Noise. (b) Component 2: Signal. 


\subsection{Hippocampal Formation Segmentation}

Segmentation of the hippocampal formation was performed using FreeSurfer tools. FreeSurfer takes about 10-12 hours for cortical reconstruction along with hippocampal segmentation of each $T_{1}$-weighted scan. The segmented hippocampal formation overlaid on the $T_{1}$ weighted MR image are shown in Figures 5.3 and 5.4. The participant number is indicated on the top left corner of each image. For each participant, the segmented hippocampal formation is shown in the axial (top) and coronal (bottom) slices passing through the medial temporal lobe.
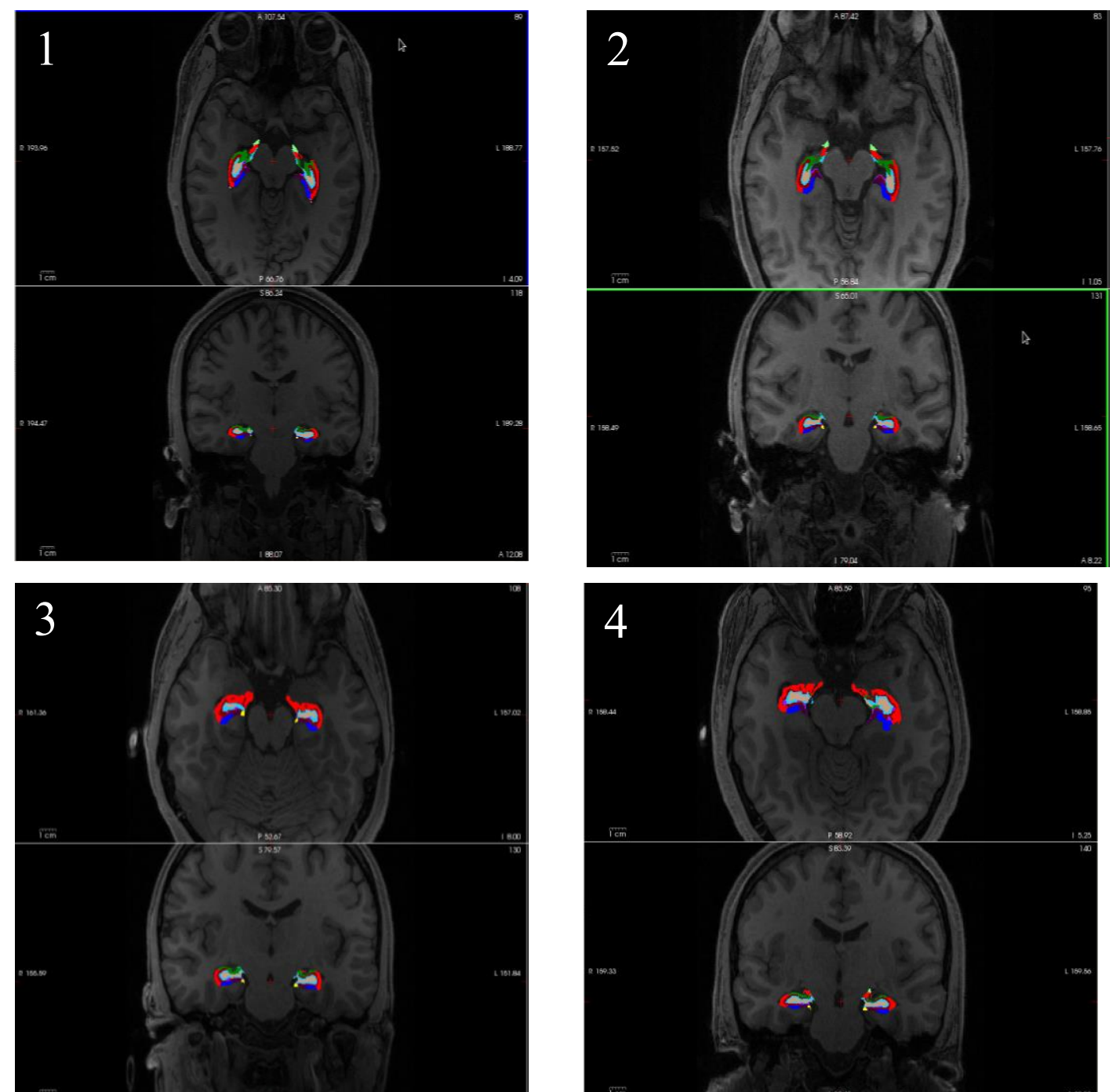

Figure 5.3: Segmented hippocampal formation for participants 1 through 4 overlaid on a $T_{1}$ - weighted MR image. Blue, red, green and cyan colors indicate the subiculum, CA1, CA3 and dentate gyrus subfields respectively. 

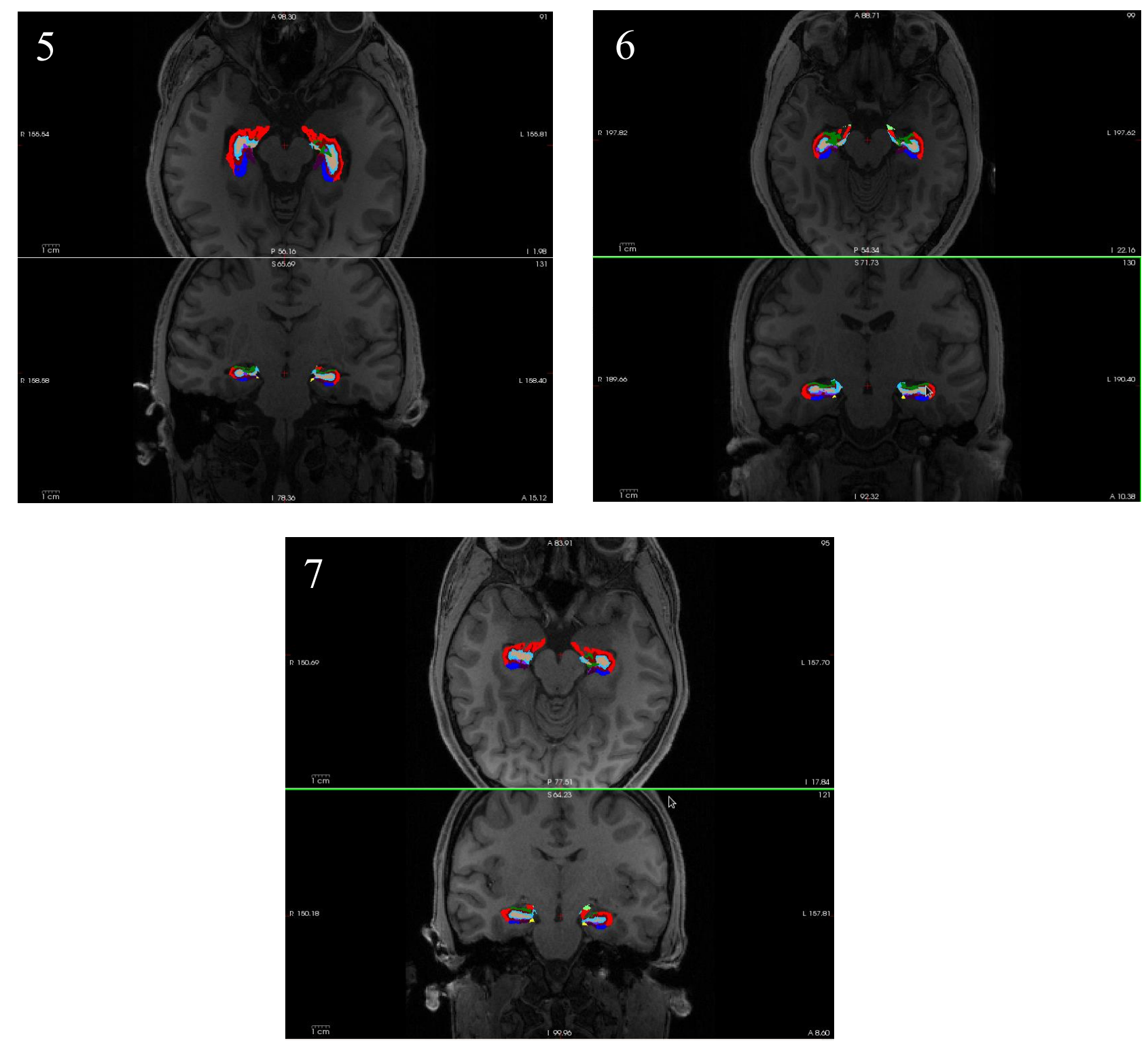

Figure 5.4: Segmented hippocampal formation for participants 5 through 7 overlaid on a $T_{1}$-weighted MR image. Blue, red, green and cyan colors indicate the subiculum, CA1, CA3 and dentate gyrus subfields respectively. 


\subsection{Cortical Reconstruction}

The entorhinal cortex, perirhinal cortex and parahippocampal cortex labels are obtained through cortical parcellation of the $T_{1}$ - weighted image using FreeSurfer tools. These cortical labels are overlaid on an inflated left and right hemisphere of each participant's brain as shown in Figure 5.5, 5.6. The participant number is indicated on the top left corner of each image.

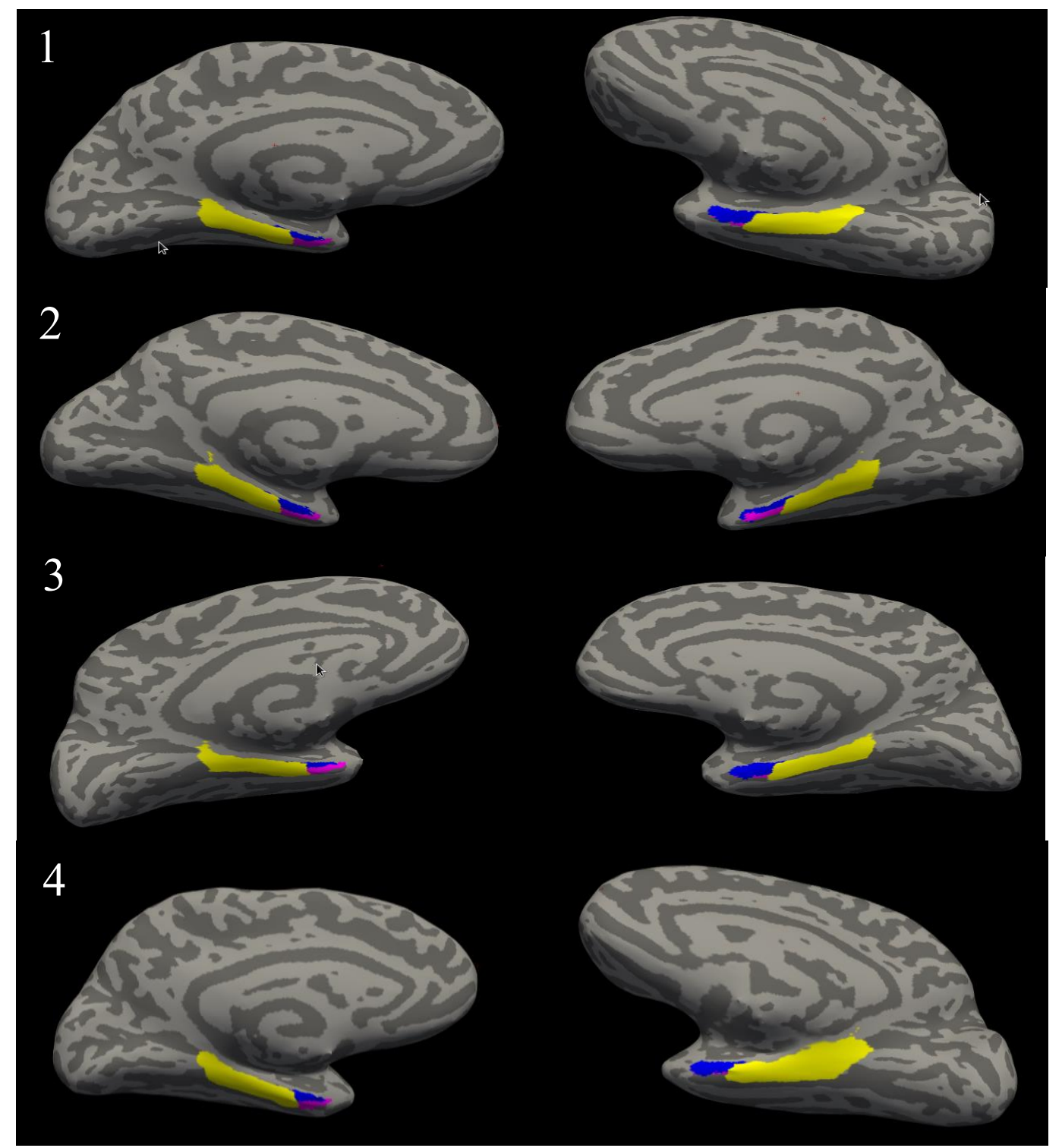

Figure 5.5: Cortical labels for participants 1 through 4, overlaid on the left (left panel) and right (right panel) inflated hemispheres. Yellow, blue and magenta colors indicate the parahippocampal, entorhinal and perirhinal cortices respectively. 


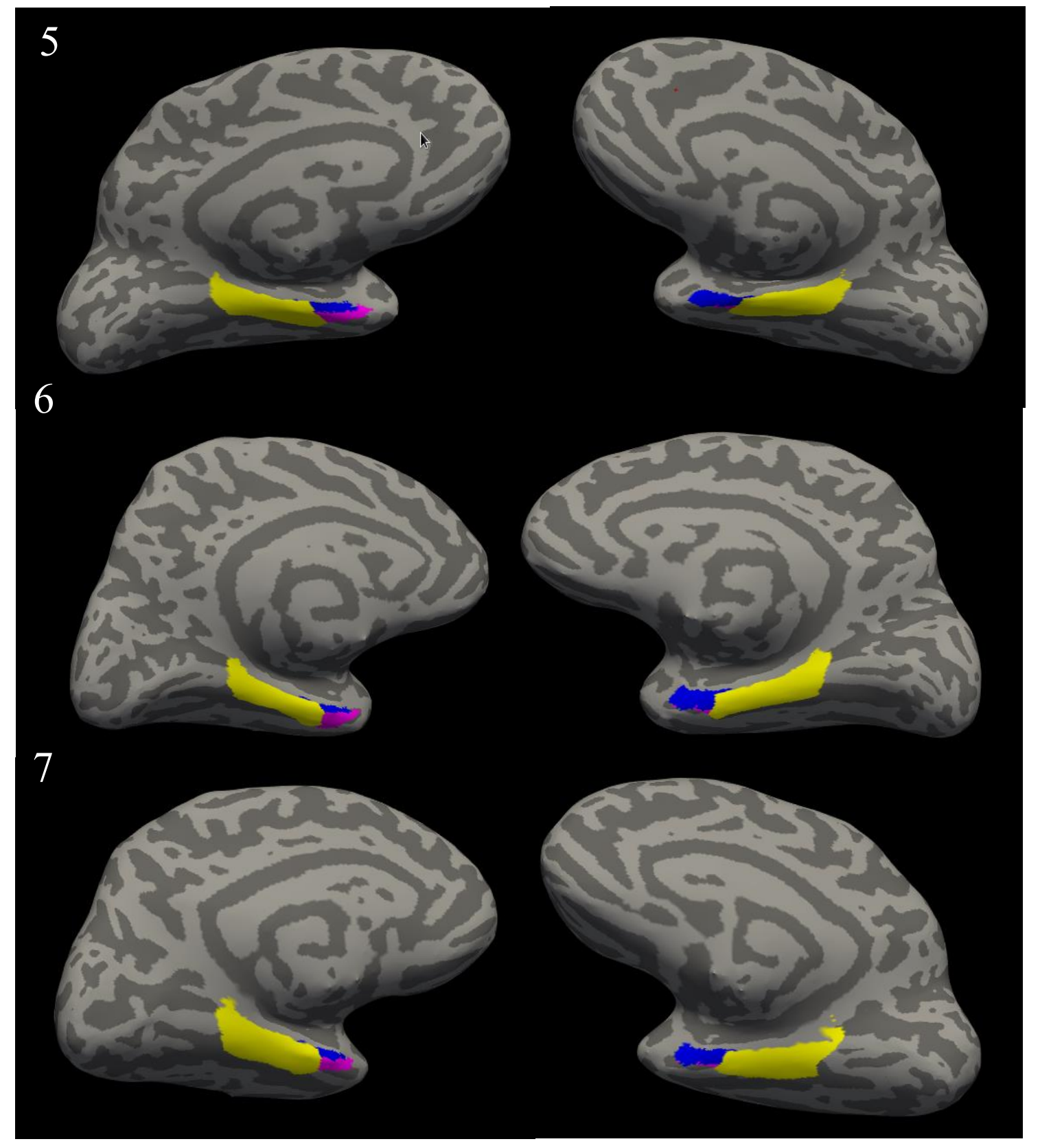

Figure 5.6: Cortical labels for participants 5 through 7, overlaid on the left (left panel) and right (right panel) inflated hemispheres. Yellow, blue and magenta colors indicate the parahippocampal, entorhinal and perirhinal cortices respectively. 


\subsection{ANTs Registration}

The cortical labels and hippocampal subfields that are obtained from the parcellation and segmentation of $T_{1}$-weighted image respectively are aligned to the low-resolution functional image. This transformation of the labels and subfields to the functional image is implemented using ANTs tools. The transformed hippocampal subfields overlaid on the functional image is shown in Figure. 5.7.

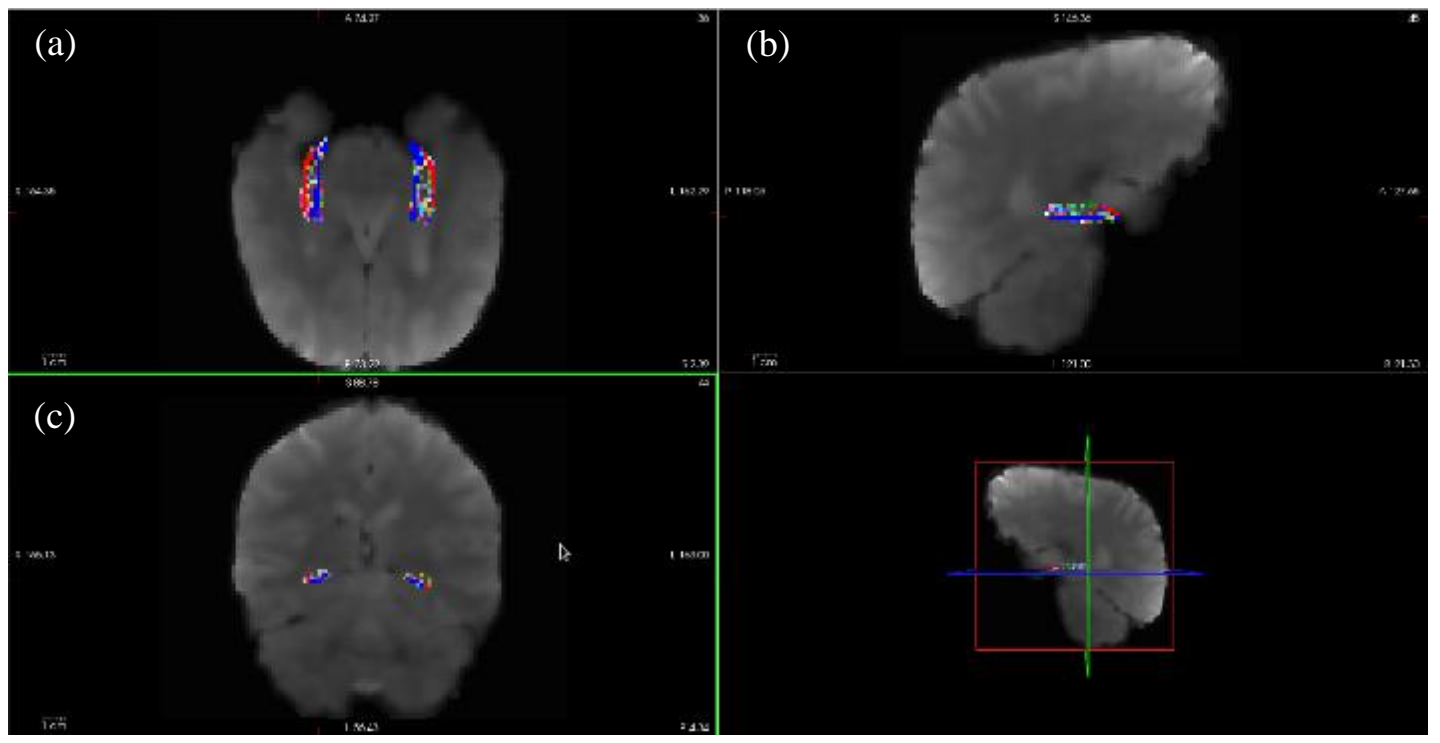

Figure 5.7: The hippocampal subfields after registration to the mean functional scan $T_{2}^{*}$ as seen in the (a) axial (b) sagittal and (c) coronal slices. 


\subsection{Hemodynamic Response Functions}

The optimized parameters of the hemodynamic response functions (HRF) of the different hippocampal and parahippocampal substructures were estimated using the algorithm described in section 4.6. The cross-validation result for one of the voxels is shown in Figure 5.8. It explicitly shows how the functional form of the HRF changes for the average curvature $\mu=\int_{0}^{30 s}\left|\frac{\partial^{2} h r f(t)}{\partial t^{2}}\right| d t$. For three different values of $\mu=2.7,4.5,7.1$ the functional form of the HRF is shown in figures A, B and C. The best HRF has two maximas and minimum. Therefore, the best estimate of HRF is given by the parameters that minimize the optimization criterion.

The hemodynamic response functions for the left and right MTL substructures are plotted for individual subjects in Figures 5.9-16. Note that the hemodynamic response functions are variance normalized. For each hemodynamic response function one of the important characteristics, the time taken to attain maximum amplitude or referred to here as the time-to-peak, was also computed and compared using a bar graph.

The uncertainties in the function HRF were determined using propagation of error rule. Since HRF is a combination of non-linear variables $a_{2}, a_{3}$ and $a_{4}$ propagation of error can be given by Taylor series expansion. The standard deviation in the function HRF is given by:

$$
s_{h r f}=\sqrt{\left(\frac{\partial h r f}{\partial a_{2}}\right)^{2} s_{a_{2}}^{2}+\left(\frac{\partial h r f}{\partial a_{3}}\right)^{2} s_{3}^{2}+\left(\frac{\partial h r f}{\partial a_{4}}\right)^{2} s_{a_{4}}^{2}}
$$

where $s_{h r f}$ represents the standard deviation or uncertainty of the function HRF, $s_{a_{2}}$ represents the standard deviation of variable $a_{2}, s_{a_{3}}$ represents the standard deviation of variable $a_{3}$, and $s_{a_{4}}$ represents the standard deviation of variable $a_{4}$. Table 5.1 summarizes the average time-to-peak (time delay in achieving maximum amplitude) for the sub-regions and the associated uncertainties of the hemodynamic response function at the time point when the peak amplitude occurs. 

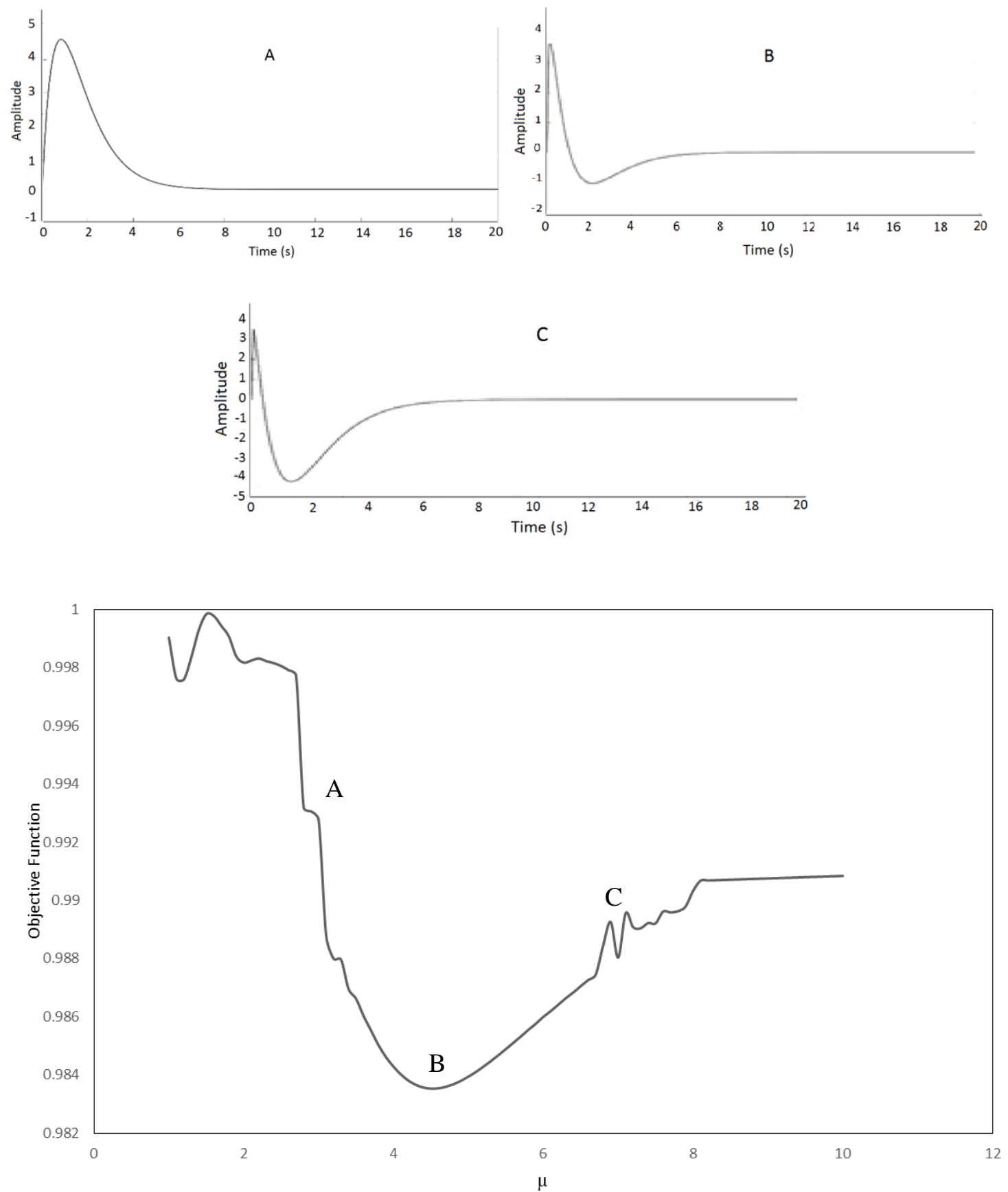

Figure 5.8: Result for the validation step of one of the voxels. The best HRF is obtained at the minimum of the objective function $(\mu=4.5)$. The graph depicts how the functional form of the HRF changes with $\mu$. Three hemodynamic response functions are shown above the graph corresponding to $\mu=2.7$ (small figure A), $\mu=4.5$ (small figure B) and $\mu=7.1$ (small figure $C$ ). 
(a)

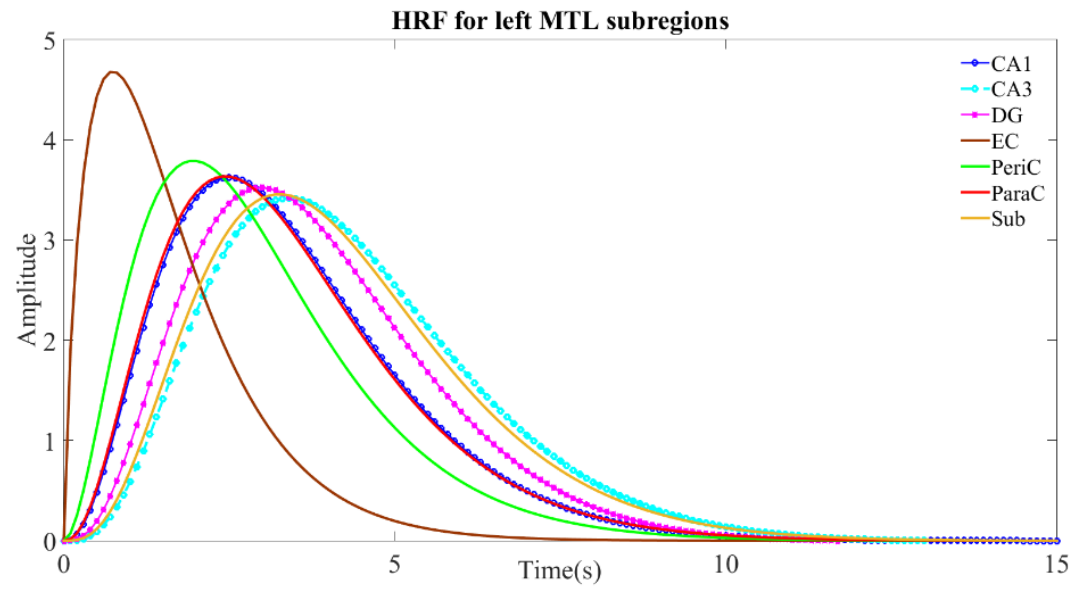

(b)

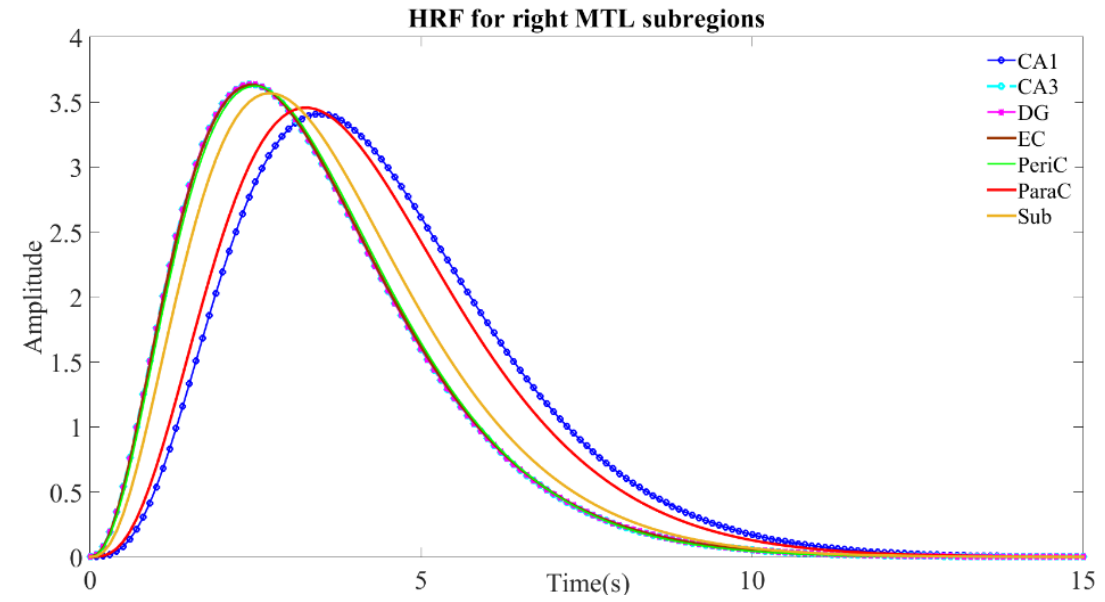

(c)

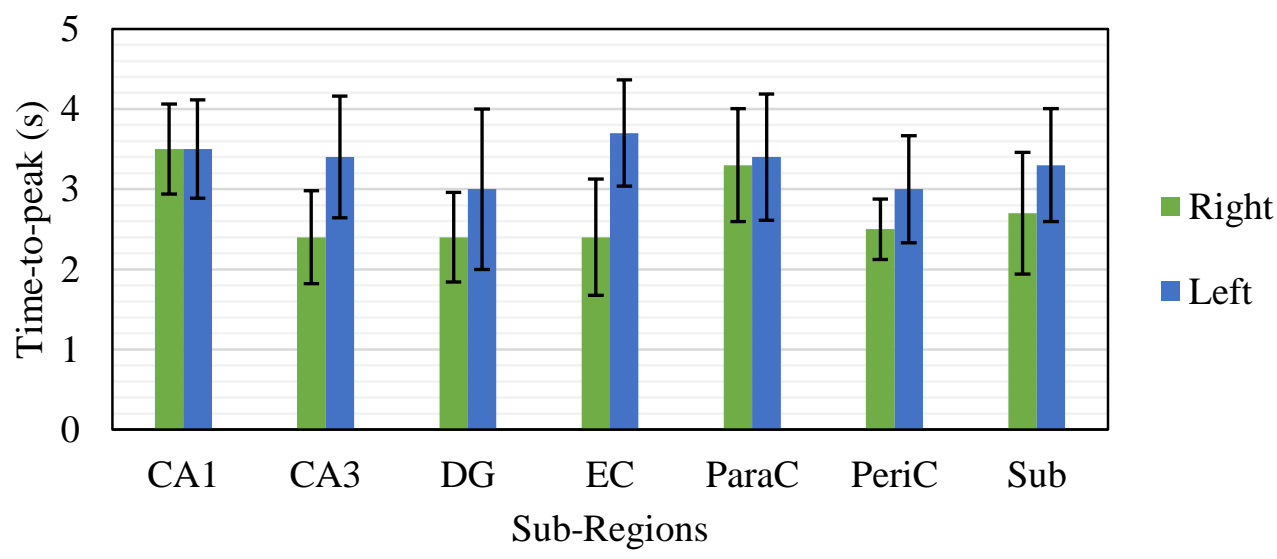

Figure 5.9: The physiological hemodynamic response functions averaged across all voxels within a subregion from both runs for participant \#1. (a) HRF for right medial temporal lobe structures. (b) HRF for left medial temporal lobe structures. (c) Comparison of the average time-to-peak of the HRF and error bars representing its associated standard deviation. 
(a)

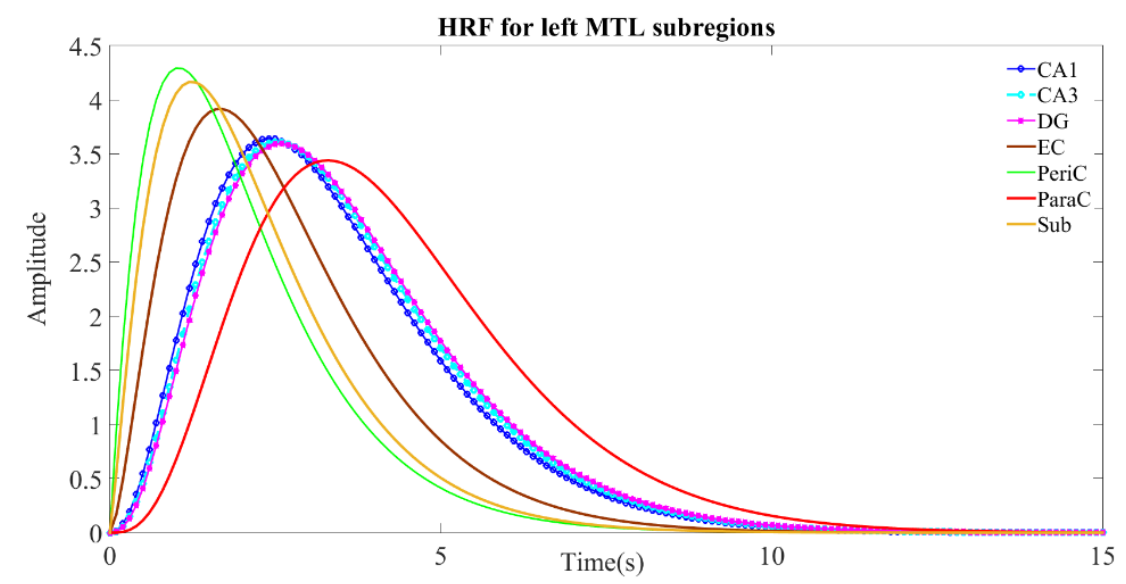

(b)

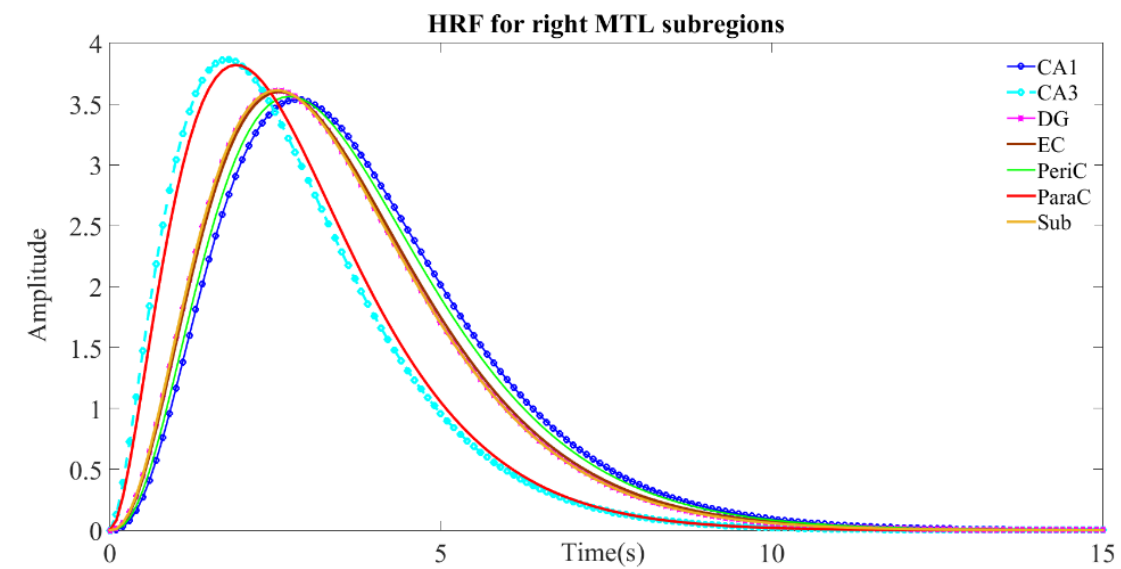

(c)

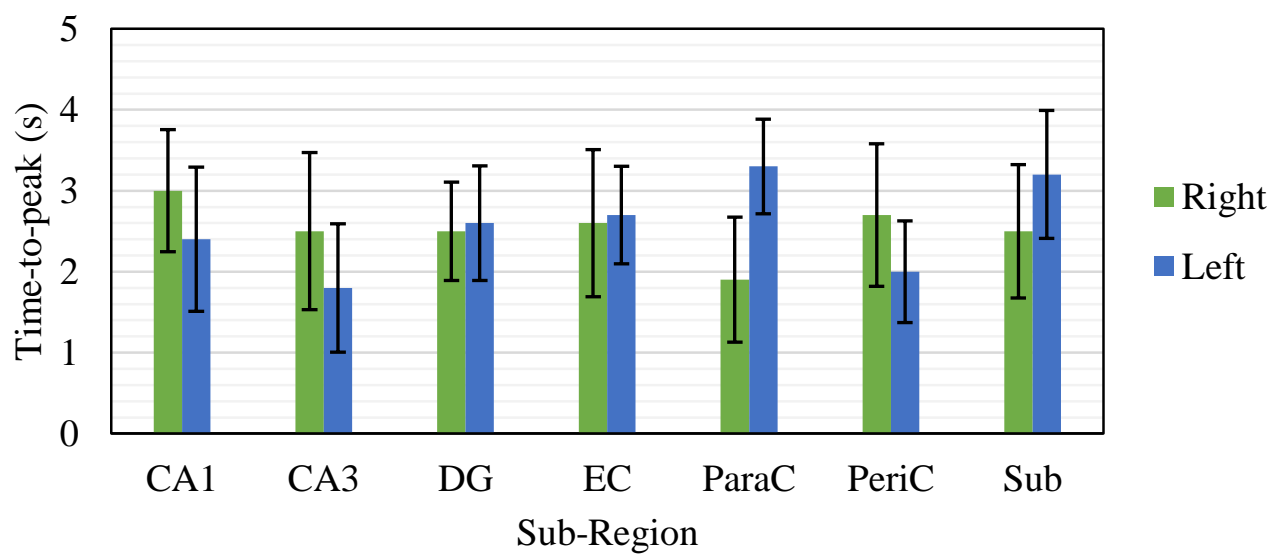

Figure 5.10: The physiological hemodynamic response functions averaged across all voxels within a subregion from both runs for participant \#2. (a) HRF for right medial temporal lobe structures. (b) HRF for left medial temporal lobe structures. (c) Comparison of the average time-to-peak of the HRF and error bars representing its associated standard deviation. 
(a)

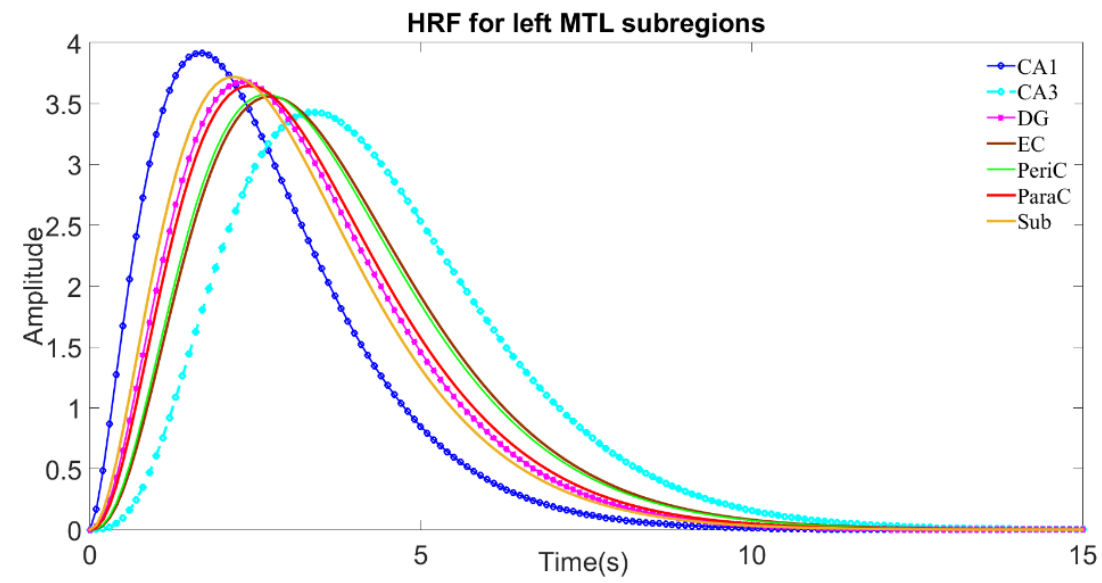

(b)

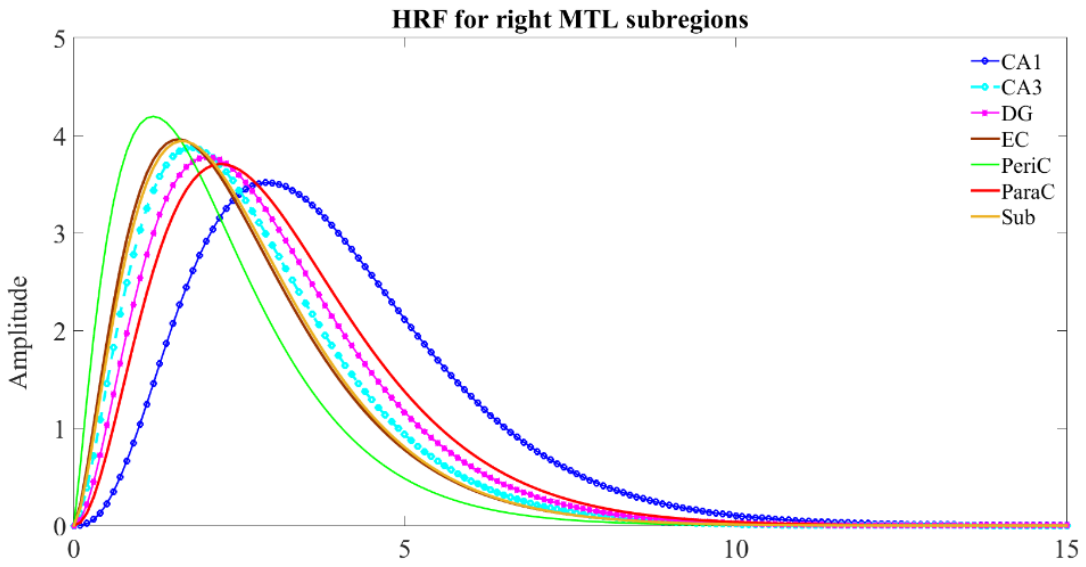

(c)

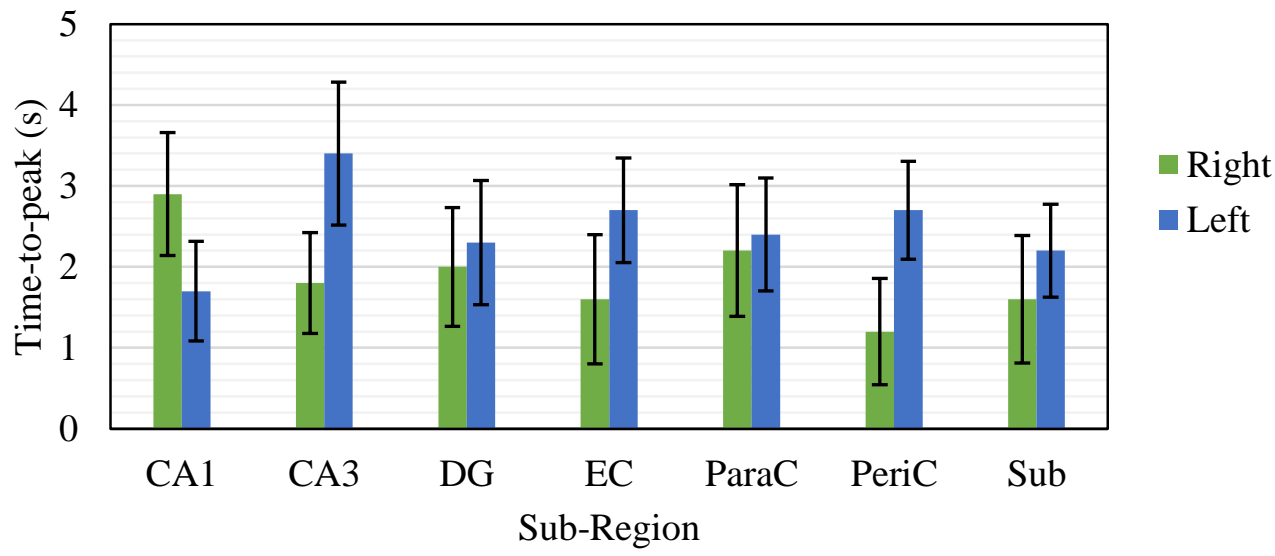

Figure 5.11: The physiological hemodynamic response functions averaged across all voxels within a subregion from both runs for participant \#3. (a) HRF for right medial temporal lobe structures. (b) HRF for left medial temporal lobe structures. (c) Comparison of the average time-to-peak of the $\mathrm{HRF}$ and error bars representing its associated standard deviation. 
(a)

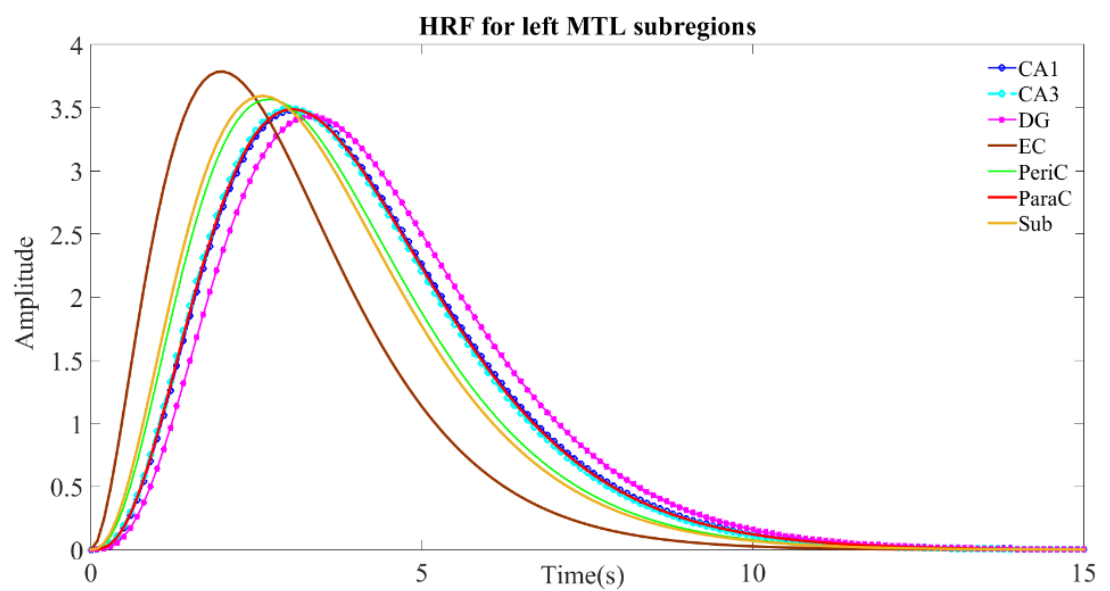

(b)

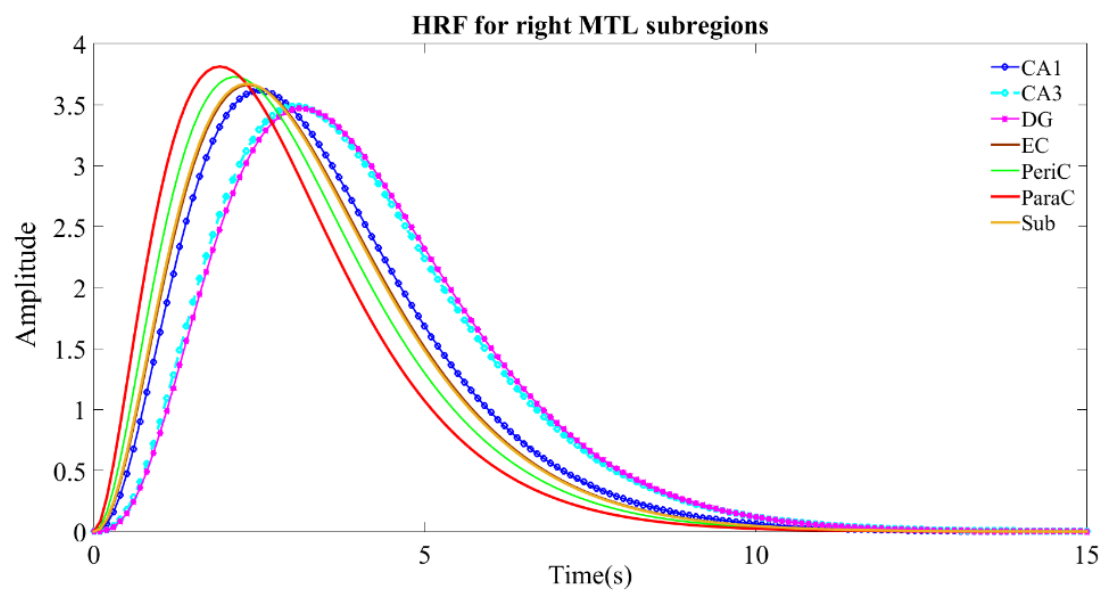

(c)

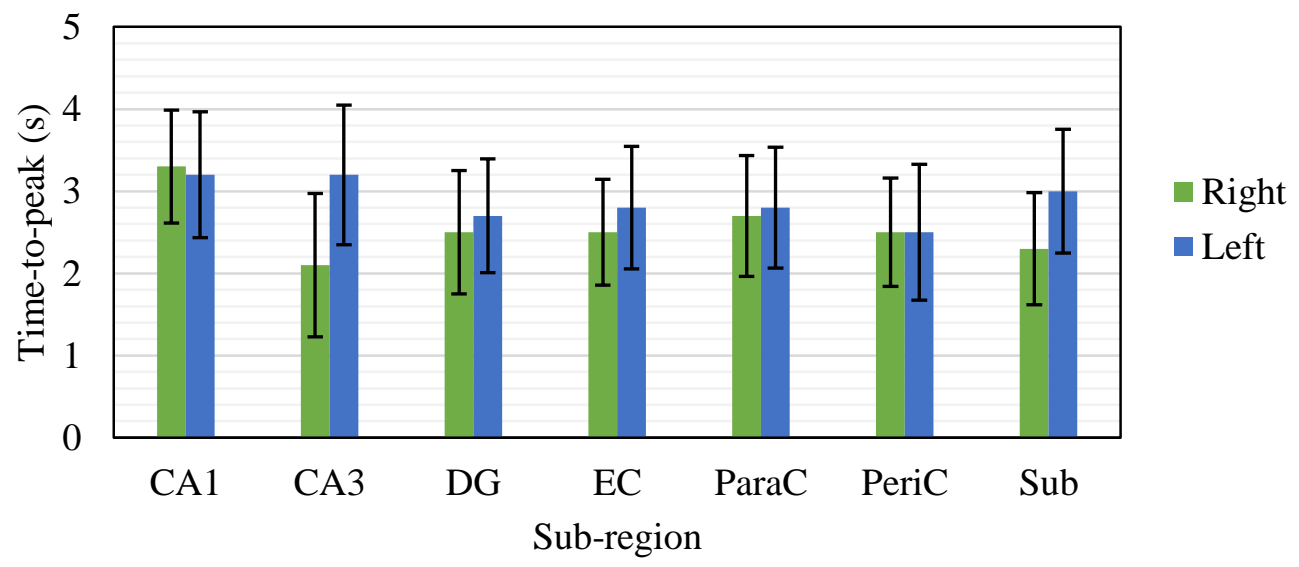

Figure 5.12: The physiological hemodynamic response functions averaged across all voxels within a subregion from both runs for participant \#4. (a) HRF for right medial temporal lobe structures. (b) HRF for left medial temporal lobe structures. (c) Comparison of the average time-to-peak of the HRF and error bars representing its associated standard deviation. 
(a)

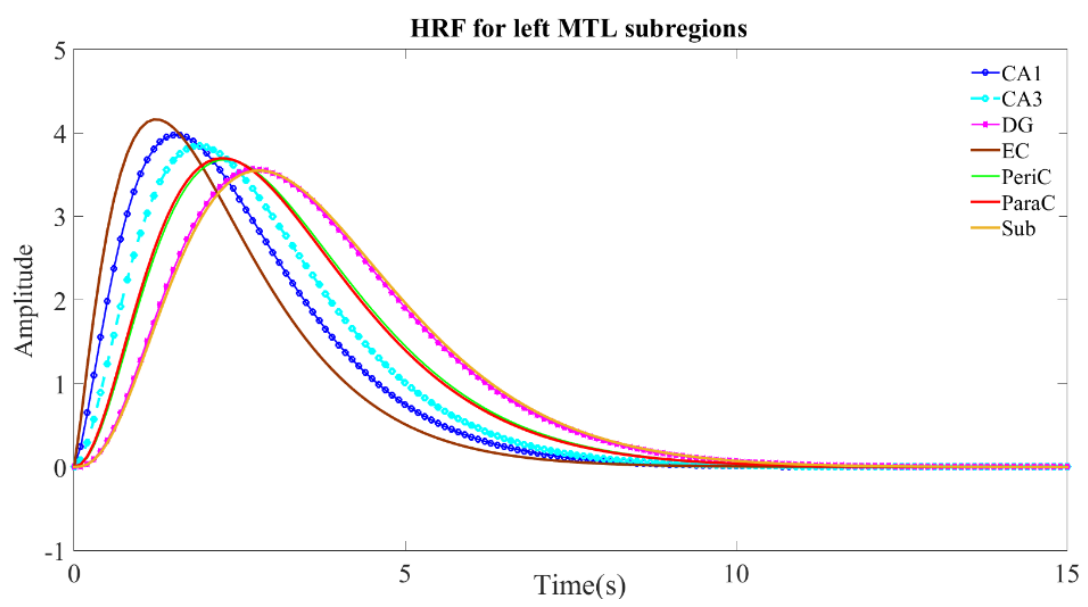

(b)

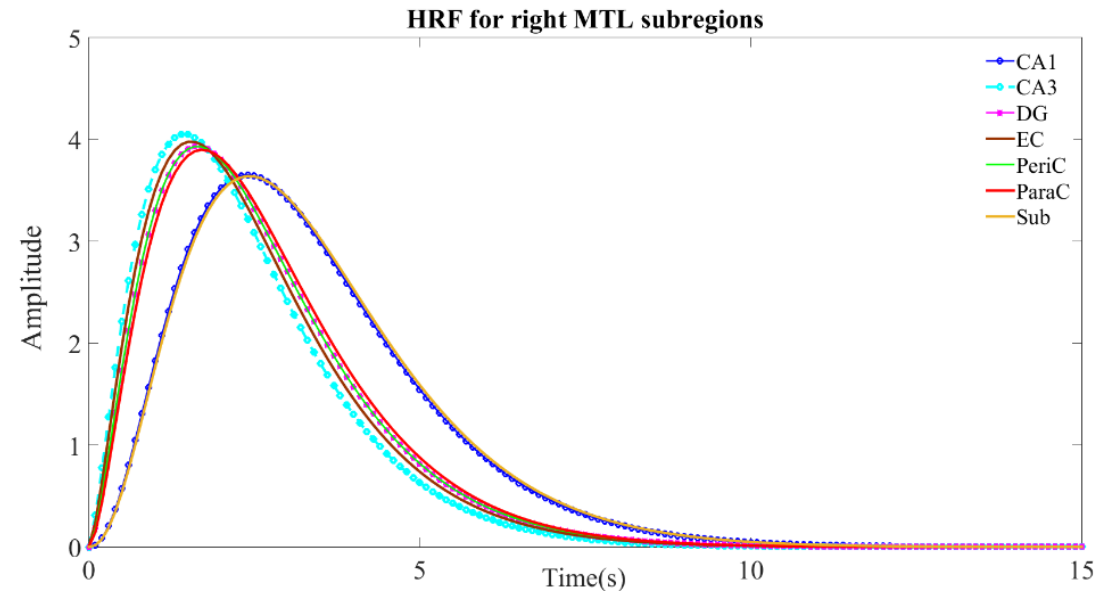

(c)

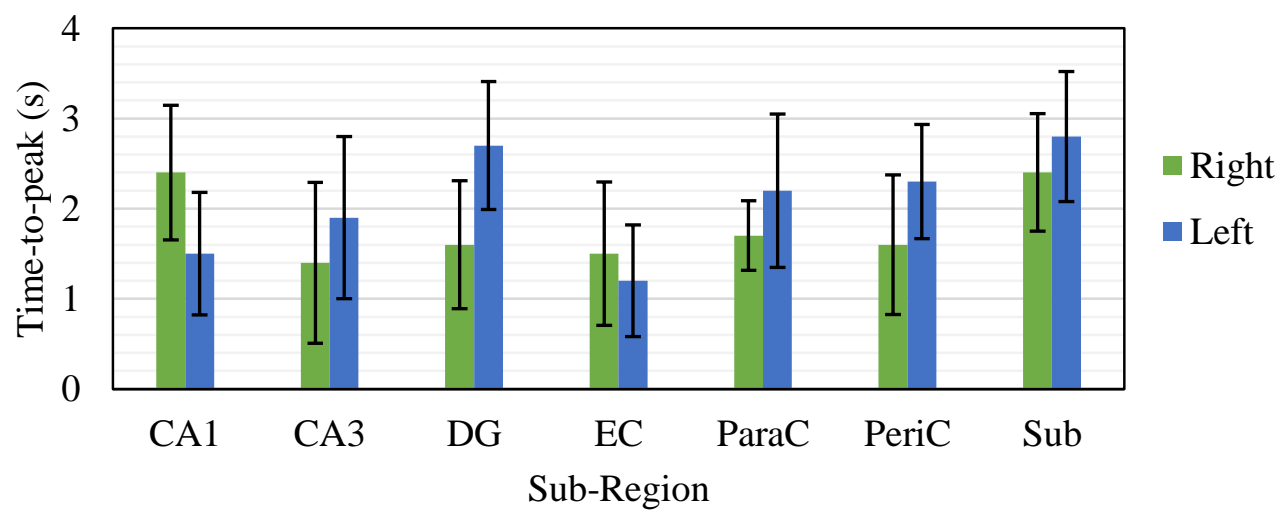

Figure 5.13: The physiological hemodynamic response functions averaged across all voxels within a subregion from both runs for participant \#5. (a) HRF for right medial temporal lobe structures. (b) HRF for left medial temporal lobe structures. (c) Comparison of the average time-to-peak of the $\mathrm{HRF}$ and error bars representing its associated standard deviation. 
(a)

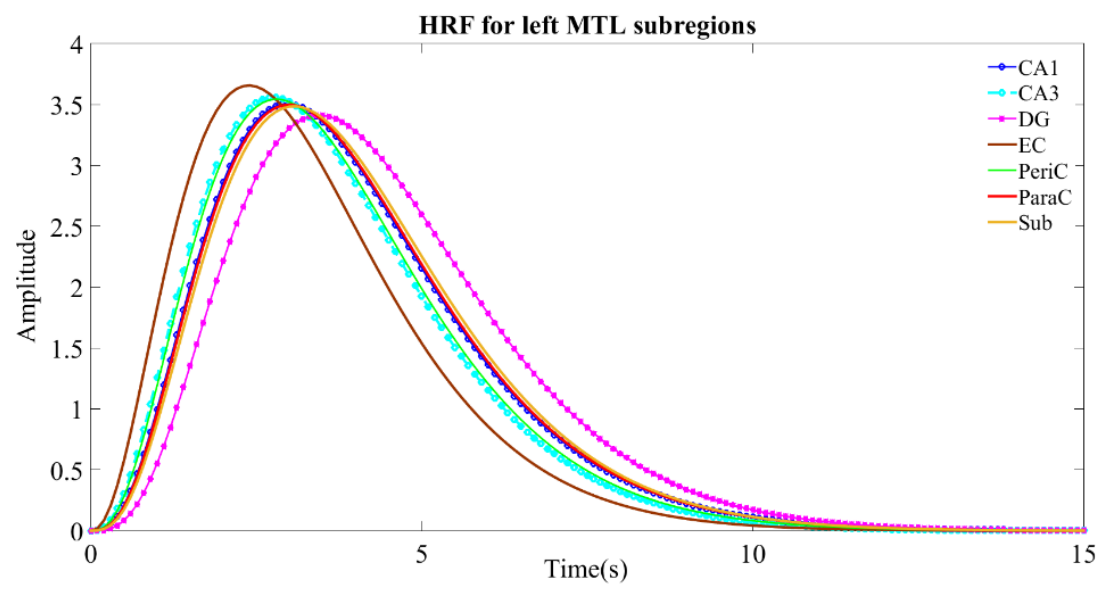

(b)

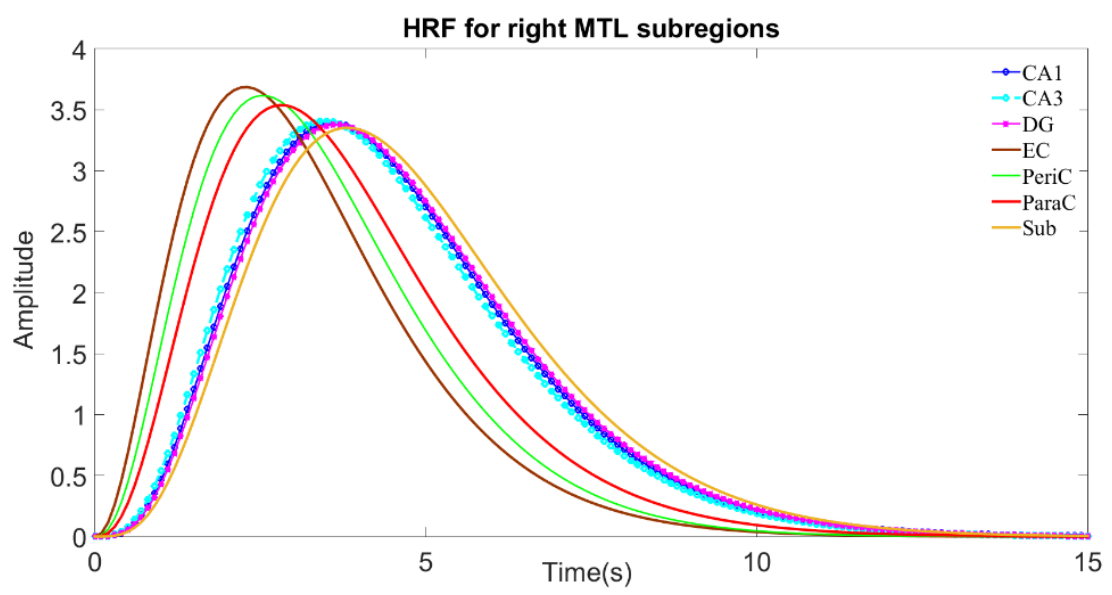

(c)

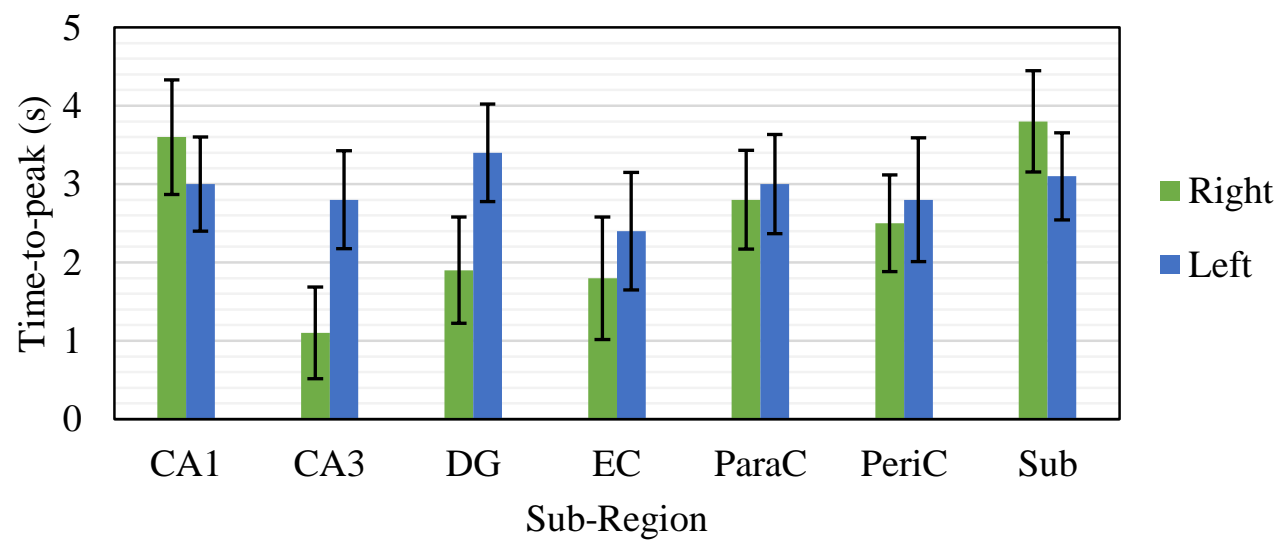

Figure 5.14: The physiological hemodynamic response functions averaged across all voxels within a subregion from both runs for participant \#6. (a) HRF for right medial temporal lobe structures. (b) HRF for left medial temporal lobe structures. (c) Comparison of the average time-to-peak of the HRF and error bars representing its associated standard deviation. 
(a)

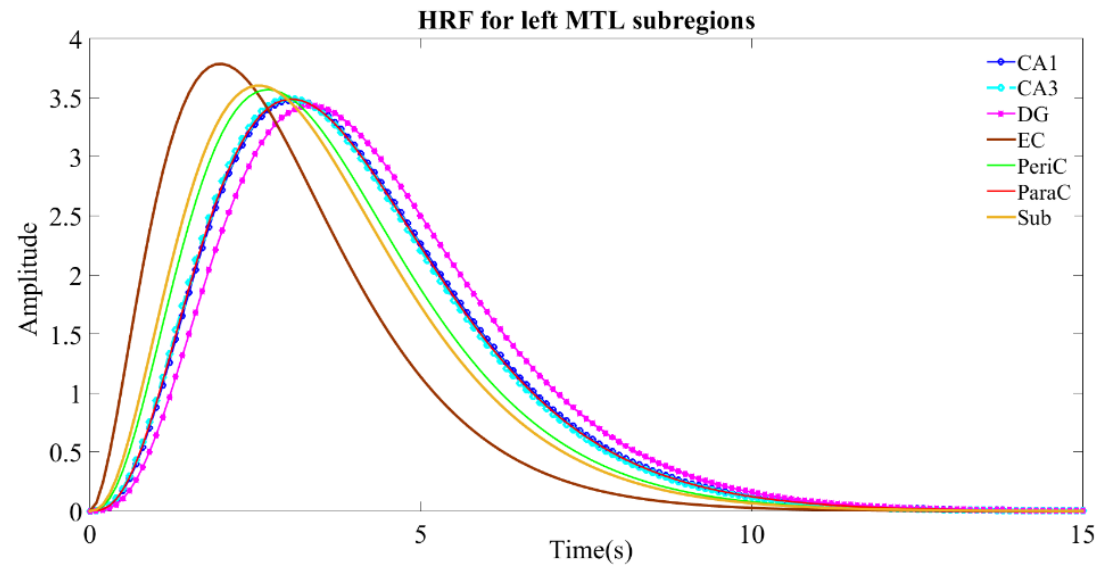

(b)

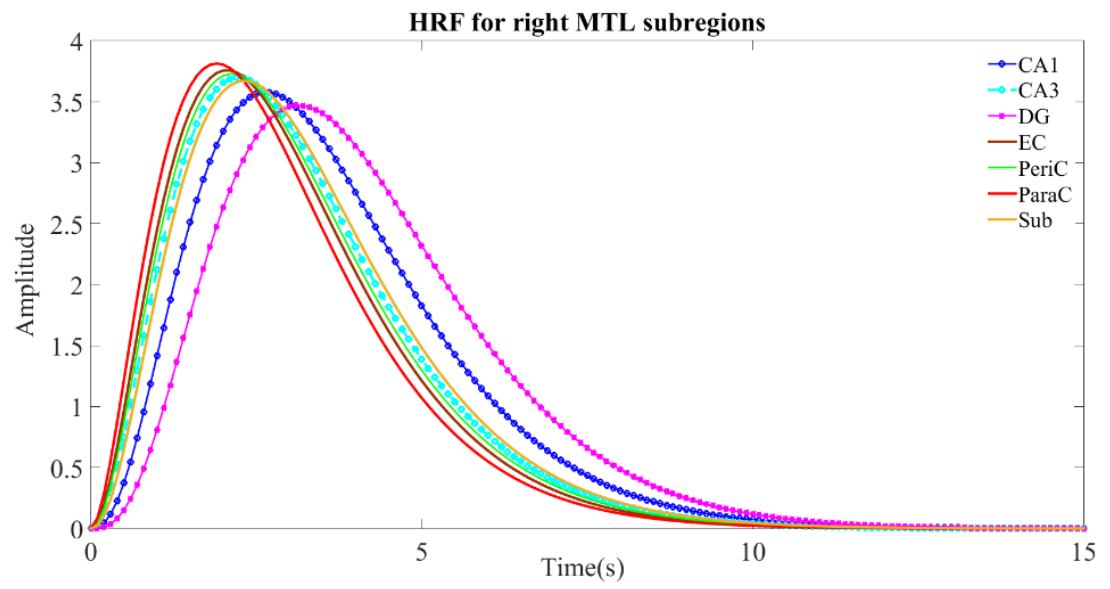

(c)

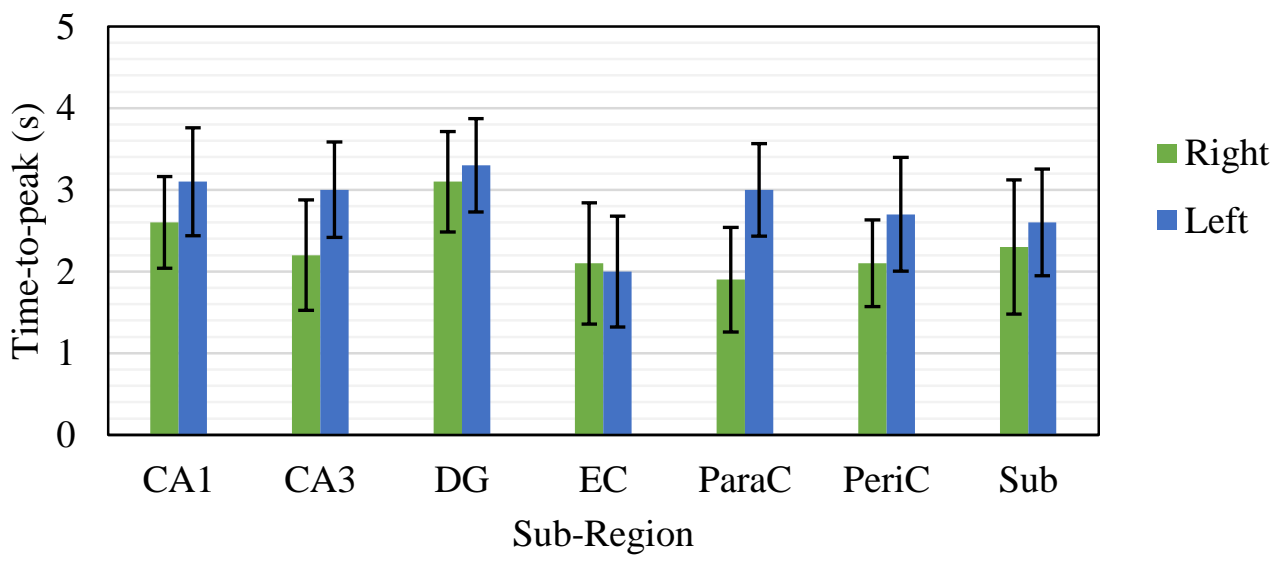

Figure 5.15: The physiological hemodynamic response functions averaged across all voxels within a subregion from both runs for participant \#7. (a) HRF for right medial temporal lobe structures. (b) HRF for left medial temporal lobe structures. (c) Comparison of the average time-to-peak of the HRF and error bars representing its associated standard deviation. 
Table 5.1: Summary of time-to-peak (in seconds) for the left and right sub-regions and their associated propagated uncertainties calculated using the Equation 5.1.

\begin{tabular}{|c|c|c|c|c|c|c|c|c|}
\hline Participant\# & & CA1 & CA3 & DG & PeriC & ParaC & EntoC & Sub \\
\hline \multirow{2}{*}{1} & $\mathbf{R}$ & $3.5 \pm 0.082$ & $2.4 \pm 0.088$ & $2.4 \pm 0.088$ & $2.4 \pm 0.088$ & $3.3 \pm 0.083$ & $2.5 \pm 0.083$ & $2.7 \pm 0.087$ \\
\hline & $\mathbf{L}$ & $3.5 \pm 0.042$ & $3.4 \pm 0.048$ & $3.0 \pm 0.065$ & $3.7 \pm 0.031$ & $3.4 \pm 0.050$ & $3.0 \pm 0.072$ & $3.3 \pm 0.048$ \\
\hline \multirow{2}{*}{2} & $\mathbf{R}$ & $3.0 \pm 0.060$ & $2.5 \pm 0.079$ & $2.5 \pm 0.076$ & $2.6 \pm 0.095$ & $1.9 \pm 0.081$ & $2.7 \pm 0.104$ & $2.5 \pm 0.077$ \\
\hline & $\mathbf{L}$ & $2.4 \pm 0.088$ & $1.8 \pm 0.124$ & $2.6 \pm 0.078$ & $2.7 \pm 0.068$ & $3.3 \pm 0.056$ & $2.0 \pm 0.115$ & $3.2 \pm 0.051$ \\
\hline \multirow{2}{*}{3} & $\mathbf{R}$ & $2.9 \pm 0.055$ & $1.8 \pm 0.083$ & $2.0 \pm 0.086$ & $1.6 \pm 0.100$ & $2.2 \pm 0.083$ & $1.2 \pm 0.102$ & $1.6 \pm 0.083$ \\
\hline & $\mathbf{L}$ & $1.7 \pm 0.087$ & $3.4 \pm 0.057$ & $2.3 \pm 0.081$ & $2.7 \pm 0.081$ & $2.4 \pm 0.079$ & $2.7 \pm 0.084$ & $2.2 \pm 0.080$ \\
\hline \multirow{2}{*}{4} & $\mathbf{R}$ & $3.3 \pm 0.049$ & $2.1 \pm 0.083$ & $2.5 \pm 0.074$ & $2.5 \pm 0.073$ & $2.7 \pm 0.074$ & $2.5 \pm 0.077$ & $2.3 \pm 0.077$ \\
\hline & $\mathbf{L}$ & $3.2 \pm 0.052$ & $3.2 \pm 0.055$ & $2.7 \pm 0.070$ & $2.8 \pm 0.062$ & $2.8 \pm 0.066$ & $2.5 \pm 0.072$ & $3.0 \pm 0.064$ \\
\hline \multirow{2}{*}{5} & $\mathbf{R}$ & $2.4 \pm 0.078$ & $1.4 \pm 0.096$ & $1.6 \pm 0.094$ & $1.5 \pm 0.098$ & $1.7 \pm 0.086$ & $1.6 \pm 0.101$ & $2.4 \pm 0.073$ \\
\hline & $\mathbf{L}$ & $1.5 \pm 0.097$ & $1.9 \pm 0.087$ & $2.7 \pm 0.065$ & $1.2 \pm 0.098$ & $2.2 \pm 0.088$ & $2.3 \pm 0.076$ & $2.8 \pm 0.063$ \\
\hline \multirow{2}{*}{6} & $\mathbf{R}$ & $3.6 \pm 0.049$ & $1.1 \pm 0.103$ & $1.9 \pm 0.098$ & $1.8 \pm 0.093$ & $2.8 \pm 0.076$ & $2.5 \pm 0.065$ & $3.8 \pm 0.039$ \\
\hline & $\mathbf{L}$ & $3.0 \pm 0.064$ & $2.8 \pm 0.112$ & $3.4 \pm 0.093$ & $2.4 \pm 0.091$ & $3.0 \pm 0.070$ & $2.8 \pm 0.076$ & $3.1 \pm 0.036$ \\
\hline \multirow{2}{*}{7} & $\mathbf{R}$ & $2.6 \pm 0.077$ & $2.2 \pm 0.097$ & $3.1 \pm 0.052$ & $2.1 \pm 0.090$ & $1.9 \pm 0.099$ & $2.1 \pm 0.092$ & $2.3 \pm 0.098$ \\
\hline & $\mathbf{L}$ & $3.1 \pm 0.060$ & $3.0 \pm 0.057$ & $3.3 \pm 0.047$ & $2.0 \pm 0.096$ & $3.0 \pm 0.067$ & $2.7 \pm 0.080$ & $2.6 \pm 0.071$ \\
\hline
\end{tabular}

An independent-samples t-test was used to determine if there were differences in the time-to-peak between CA1 and CA3 as well as between CA1 and DG. The t-test revealed that for six out of seven participants, the time taken by hemodynamic response of the CA1 subfield is significantly greater than the time taken by hemodynamic response of the CA3 subfield (Participant \#1: t $(128)=1.99, \mathrm{p}=0.024$; Participant \#2: $\mathrm{t}(142)=2.46, \mathrm{p}=0.007$; Participant \#3: $\mathrm{t}(108)=2.69, \mathrm{p}=0.005$; Participant \#4: $\mathrm{t}(244)=$ 2.38, $\mathrm{p}=0.009 ;$ Participant \#5: $\mathrm{t}(175)=4.72, \mathrm{p}=0.0002$; Participant \#6: $\mathrm{t}(142)=2.46, \mathrm{p}=0.007$; Participant $\# 6: \mathrm{t}(175)=4.72, \mathrm{p}=0.0002)$ but not for participant \#7 $(\mathrm{t}(219)=1.29, \mathrm{p}=0.09)$. Another set of $\mathrm{t}$-tests for testing difference between the time-to-peak between CA1 and DG was carried out. It was found that for five participants, the time-to-peak for CA1 was significantly higher than time-to-peak for DG (Participant $\# 1: \mathrm{t}(130)=2.08, \mathrm{p}=0.01 ;$ Participant \#3: $\mathrm{t}(186)=2.28, \mathrm{p}=0.01 ;$ Participant \#4: $\mathrm{t}(270)=2.071, \mathrm{p}=0.019$; Participant \#5: t $(215)=3.841, \mathrm{p}=0.0008$; Participant \#7: $\mathrm{t}(318)=2.393, \mathrm{p}=0.008)$ but not for two participants (Participant \#2: t $(115)=6.169, \mathrm{p}=0.269$ and Participant \#7: $\mathrm{t}(188)=1.791, \mathrm{p}=0.963)$. 


\section{Discussions \& Conclusions}

The independent components obtained after ICA that were pre-processed using FIX were also manually investigated for any possible signal components that may have been regressed out in the process. Overall, FIX performed well in eliminating the noise components while retaining the signal components and 'unknown' components. As seen from the Figures 5.3- 6 the hippocampal segmentation using FreeSurfer achieved satisfactory delineations of the CA1, CA3, DG and subiculum volumes. Furthermore, the cortical labels for entorhinal, perirhinal and parahippocampal cortex represent every location that could possibly be in either label. Unless they could be predicted perfectly, there exists a minor overlap since the labels share a border. ANTs registration was performed in an effort to transform the volumes/labels obtained from the segmentation of the $T_{1}$ anatomical scan to the $T_{2}^{*}$ functional scan. This registration allowed for obtaining the time courses of the voxels in the regions of interest for further processing.

The primary goal of this research was to compute and compare the hemodynamic response functions of the hippocampus (CA1, CA3 and DG), entorhinal cortex and the parahippocampal regions (perirhinal cortex and parahippocampal cortex) in both hemispheres between subjects as well as between regions within a subject. The optimization algorithm proposed in this thesis identified these differences in the hemodynamic responses. As observed from the plots of the hemodynamic responses in the previous chapter, the responses varied from person to person. Even though each participant performed a similar spatial memory task, their hemodynamic responses are quite different from one another. Furthermore, it was hypothesized that for an individual, the medial temporal lobe substructures may exhibit distinct hemodynamics in response to the spatial task. The results from the present study have provided evidence 
of this distinction between the hippocampal and parahippocampal regions. The hemodynamic responses of each sub-region was found to be different from the other. These findings support the idea that the hemodynamic responses are subject specific and region specific (Boynton et al., 1996; Richter, Ugurbil and Kim, 1996; Zarahn, Aguirre and D'Esposito, 1998).

An interesting feature of the hemodynamic responses that was common to all the regions and participants is that they do not possess an 'undershoot' unlike a typical hemodynamic response function discussed in section 2.4.2. Since short stimulus durations, like 3s used in this study, do not allow for pooling of oxygenated $\mathrm{Hb}$ from remote vasculature, the amount of deoxygenated $\mathrm{Hb}$ generated is small leading to no undershoot in the signal. Additionally, for a typical hemodynamic response function, the maximum amplitude occurs at about 5-6s after it has been stimulated. In contrast, the hemodynamic responses observed in this study achieved maximum amplitudes in much shorter times $(<5 \mathrm{~s})$. These characteristics of the hemodynamic responses observed suggest that the medial temporal lobe structures were greatly active during the spatial memory task.

Previous studies have established the role of the right MTL in spatial memory and the left MTL in episodic memory. The present study investigated the role of the right MTL in a spatial memory task. This was determined by comparing the hemodynamic responses of the left MTL substructures to those of the right MTL substructures. In most cases, it was found that the right MTL substructures achieved maximum amplitude in times shorter than the left medial temporal lobe structures. This indicates that the right MTL substructures achieved overall higher firing rates than the left MTL substructures. This behaviour of the left hippocampal and parahippocampal regions further corroborated with the previous findings that suggest that the left MTL structures may be not be supporting spatial memory. Moreover, these outcomes suggest the substantial involvement of the right hippocampal and parahippocampal sub-regions in spatial memory processing. The right MTL substructures operate in conjunction with other networks and brain structures. Therefore, any claim that the MTL structures are required for spatial memory does not mean that other brain areas are not involved as well. 
A study conducted by Bakker and colleagues in 2008 showed that the CA3/DG subfields of the hippocampus are more likely to fully remap than CA1 across similar environments (pattern separation like signal). Therefore, it is hypothesized that the CA3 and DG sub-regions fire at rates higher than the CA1 sub-region. In the present study, the hemodynamic responses of the CA3/DG sub-regions are compared with the CA1 sub-regions for each subject to quantify the differential activity. In particular, the time taken by the hemodynamic responses of the sub-regions to attain maximum amplitude was compared. In most cases, a t-test revealed statistically significant differences between the CA1 and CA3 sub-regions as well as CA1 and DG sub-regions. These findings confirm that the firing rates of CA3/DG are higher than the CA1 subfield during spatial memory tasks.

By virtue of its anatomy, the entorhinal cortex acts as a neocortical 'gate-keeper'. It receives very highly processed multimodal input from the neocortical regions (perirhinal and parahippocampal cortex in particular) and relays synaptic information from the hippocampal formation. The entorhinal cortex, perirhinal cortex and parahippocampal cortex are closely associated to the hippocampal formation. Therefore the findings that the hippocampal formation is crucially involved in spatial memory processes suggests that this should also be the case for the entorhinal cortex, perirhinal cortex and parahippocampal cortex. Although the specific functional contributions of these cortical regions to spatial memory remain to be established, they are most likely different from, but complementary to those of the hippocampus. As seen from the plots of hemodynamic responses in the previous chapter the right entorhinal, perirhinal and parahippocampal cortices demonstrated characteristics similar to those of the hippocampal formation. These findings promote the hypothesized involvement of the right entorhinal, perirhinal and parahippocampal cortex in spatial memory and are convergent with the findings of previous studies (Sargolini et al., 2006; Pihlajamaki et al., 2004; Ploner et al., 2000).

The ability to remember and navigate spatial environments is critical for everyday life. So far it has been established that the hippocampus and its surrounding cortical structures are crucial for spatial memory. Studies in the past have shown that the DG subfield is particularly vulnerable to the effects of 
aging (West, 1993) and the dysfunction of the hippocampus is one of the first symptoms of AD (e.g. Kolb and Wishaw, 1996). Therefore comprehending the functions of these structures is important. The present research is an attempt to understand the underlying physiological processes of the MTL substructures. The findings of this research may allow us a greater understanding of how the human brain actually works. Furthermore, we can take this basic understanding of the brain and use it for the future treatment of neurological disorders.

A strength of this research is that the computed hemodynamic response functions were derived using an optimization algorithm implementing a cross validation approach instead of a convolution approach. The hemodynamic response function was parametrized with forms previously established and the parameters of the hemodynamic response function were allowed all possible values. A second order derivative and constraints were explicitly introduced to allow for subject specific variations of the hemodynamic response function. Each time course was split into two datasets and each dataset was used once for estimation and once for validation. The average from the two approaches thus provided the most optimal estimation of parameters of the hemodynamic response function.

One of the limitations of this study is that only 7 out of 24 participants that were scanned were included in the analysis of the study. A greater sample size would have given greater power to detect differences. However, the remaining 17 participants had to be eliminated either due to poor performance during the spatial memory paradigm and/or due to a great deal of head motion during scanning that negatively impacted the image quality. Secondly, the participants chosen for the study belonged to a narrow age group. The reason for this was since the scanning was carried out at the MRI facility at the University of Colorado, Boulder, it was convenient to recruit university students. Therefore the participants chosen for this study belonged to the 21- 30 year age group. Due to the exclusion of the elderly in this study I was unable to assess the impact of age on spatial memory performance. Lastly, the relationship of the left MTL structures in episodic memory were unexplored. An additional paradigm for episodic memory may have aided in understanding the physiological hemodynamics of the left MTL substructures, however this would 
require additional scanning time which in turn would increase the problem associated with subject motion and also be expensive.

As discussed in this thesis the right hippocampal formation and its associated structures are central to spatial memory. Now that our insights into the physiological hemodynamics of the hippocampal and parahippocampal regions are much more detailed, a future step would be to perform dynamic functional connectivity analysis, a technique to probe the relationship between spatially separated brain areas, in order to understand the integrated relationship between these structures.

To summarize, the optimization algorithm proposed allows for estimation of the hemodynamic characteristics from the BOLD signal. This study shows that the parahippocampal regions and the hippocampal formation are critically involved in spatial memory. The differentiation of the hemodynamic function between the regions of the hippocampal formation and adjacent parahippocampal structures is demonstrated in this work. Decoding the physiological processes has revealed the fundamental characteristics of the MTL substructures in response to a specific spatial memory task.

In conclusion, this research found evidence for the predicted difference between the time-to-peak characteristic of the hemodynamic response function of the MTL structures to a specific spatial memory task. These results are also consistent with a theoretical view which holds that the right MTL substructures play a crucial role in spatial memory. Additionally, it was also found that the hemodynamic response functions are different for different individuals. 


\section{Appendix}

Appendix A: The accuracy (percent correct responses) and response time (in seconds).

\begin{tabular}{|c|c|c|c|}
\hline Subject ID & Run & Accuracy (\%) & Response Time (s) \\
\hline \multirow[t]{2}{*}{ 0 } & 1 & 72.23 & 1.522 \\
\hline & 2 & 74.23 & 1.364 \\
\hline \multirow[t]{2}{*}{1} & 1 & 82.67 & 1.693 \\
\hline & 2 & 87.56 & 1.595 \\
\hline \multirow[t]{2}{*}{2} & 1 & 47.56 & 2.172 \\
\hline & 2 & 60.67 & 1.9 \\
\hline \multirow[t]{2}{*}{5} & 1 & 72.45 & 1.738 \\
\hline & 2 & 82 & 1.654 \\
\hline \multirow[t]{2}{*}{7} & 1 & 63.78 & 1.738 \\
\hline & 2 & 61.11 & 1.31 \\
\hline \multirow[t]{2}{*}{8} & 1 & 65.56 & 2.159 \\
\hline & 2 & 51.78 & 2.027 \\
\hline \multirow[t]{2}{*}{13} & 1 & 63.34 & 1.782 \\
\hline & 2 & 47.12 & 1.67 \\
\hline \multirow[t]{2}{*}{15} & 1 & 74 & 1.903 \\
\hline & 2 & 77.78 & 1.683 \\
\hline \multirow[t]{2}{*}{16} & 1 & 64.23 & 1.535 \\
\hline & 2 & 66.45 & 1.424 \\
\hline \multirow[t]{2}{*}{20} & 1 & 67.56 & 1.914 \\
\hline & 2 & 76.45 & 1.771 \\
\hline \multirow[t]{2}{*}{30} & 1 & 70.45 & 1.863 \\
\hline & 2 & 71.78 & 1.76 \\
\hline \multirow[t]{2}{*}{33} & 1 & 67.56 & 1.509 \\
\hline & 2 & 76.89 & 1.539 \\
\hline
\end{tabular}




\begin{tabular}{|c|c|c|c|}
\hline Subject ID & Run & Accuracy (\%) & Response Time (s) \\
\hline \multirow[t]{2}{*}{36} & 1 & 75.11 & 2.259 \\
\hline & 2 & 76.44 & 2.04 \\
\hline \multirow[t]{2}{*}{39} & 1 & 64.67 & 1.626 \\
\hline & 2 & 68.22 & 1.574 \\
\hline \multirow[t]{2}{*}{44} & 1 & 66 & 1.658 \\
\hline & 2 & 65.34 & 1.582 \\
\hline \multirow[t]{2}{*}{47} & 1 & 69.11 & 2.1 \\
\hline & 2 & 70 & 1.71 \\
\hline \multirow[t]{2}{*}{52} & 1 & 54.67 & 2.175 \\
\hline & 2 & 69.56 & 2.134 \\
\hline \multirow[t]{2}{*}{58} & 1 & 75.11 & 1.817 \\
\hline & 2 & 74 & 1.754 \\
\hline \multirow[t]{2}{*}{70} & 1 & 57.78 & 1.744 \\
\hline & 2 & 66.89 & 1.693 \\
\hline \multirow[t]{2}{*}{85} & 1 & 63.55 & 1.778 \\
\hline & 2 & 72.22 & 1.588 \\
\hline \multirow[t]{2}{*}{89} & 1 & 73.12 & 1.942 \\
\hline & 2 & 78 & 1.607 \\
\hline \multirow[t]{2}{*}{131} & 1 & 75.12 & 1.691 \\
\hline & 2 & 80.89 & 1.762 \\
\hline \multirow[t]{2}{*}{141} & 1 & 72.67 & 1.487 \\
\hline & 2 & 78.23 & 1.42 \\
\hline \multirow[t]{2}{*}{144} & 1 & 58.67 & 1.67 \\
\hline & 2 & 74.23 & 1.572 \\
\hline
\end{tabular}




\section{References}

Abrahams, S., Pickering, A., Polkey, C. and Morris, R. (1997). Spatial memory deficits in patients with unilateral damage to the right hippocampal formation. Neuropsychologia, 35(1), pp.11-24.

Aguirre, G., Zarahn, E. and D'Esposito, M. (1998). The Variability of Human, BOLD Hemodynamic Responses. NeuroImage, 8(4), pp.360-369.

Andersen, P. (2007). The hippocampus book. Oxford: Oxford University Press.

Ashby, F. (2011). Statistical analysis of fMRI data. Cambridge, Mass.: MIT Press.

Avants, B., Epstein, C., Grossman, M. and Gee, J. (2008). Symmetric diffeomorphic image registration with cross-correlation: Evaluating automated labeling of elderly and neurodegenerative brain. Medical Image Analysis, 12(1), pp.26-41.

Bakker, A., Kirwan, C., Miller, M. and Stark, C. (2008). Pattern Separation in the Human Hippocampal CA3 and Dentate Gyrus. Science, 319(5870), pp.1640-1642.

Barense, M., Gaffan, D. and Graham, K. (2007). The human medial temporal lobe processes online representations of complex objects. Neuropsychologia, 45(13), pp.2963-2974.

Beckmann, C. and Smith, S. (2004). Probabilistic Independent Component Analysis for Functional Magnetic Resonance Imaging. IEEE Transactions on Medical Imaging, 23(2), pp.137-152.

Bird, C. and Burgess, N. (2008). The hippocampus and memory: insights from spatial processing.Nature Reviews Neuroscience, 9(3), pp.182-194.

Bohbot, V., Kalina, M., Stepankova, K., Spackova, N., Petrides, M. and Nadel, L. (1998). Spatial memory deficits in patients with lesions to the right hippocampus and to the right parahippocampal cortex. Neuropsychologia, 36(11), pp.1217-1238.

Boynton, G., Engel, S., Glover, G. and Heeger, D. (1996). Linear Systems Analysis of Functional Magnetic Resonance Imaging in Human V1. The Journal of Neuroscience, 16(13), pp.4207-4221.

Buffalo, E., Bellgowan, P. and Martin, A. (2006). Distinct roles for medial temporal lobe structures in memory for objects and their locations. Learning \& Memory, 13(5), pp.638-643. 
Burgess, N., Maguire, E. and O'Keefe, J. (2002). The Human Hippocampus and Spatial and Episodic Memory. Neuron, 35(4), pp.625-641.

Canto, C., Wouterlood, F. and Witter, M. (2008). What Does the Anatomical Organization of the Entorhinal Cortex Tell Us?. Neural Plasticity, 2008, pp.1-18.

Cohen, M. and Bookheimer, S. (1994). Localization of brain function using magnetic resonance imaging. Trends in Neurosciences, 17(7), pp.268-277.

Cordes, D., Nandy, R., Schafer, S. and Wager, T. (2014). Characterization and reduction of cardiac- and respiratory-induced noise as a function of the sampling rate (TR) in fMRI. NeuroImage, 89, pp.314330.

Dale, A. and Sereno, M. (1993). Improved Localizadon of Cortical Activity by Combining EEG and MEG with MRI Cortical Surface Reconstruction: A Linear Approach. Journal of Cognitive Neuroscience, 5(2), pp.162-176.

Dale, A., Fischl, B. and Sereno, M. (1999). Cortical Surface-Based Analysis. NeuroImage, 9(2), pp.179194.

Desikan, R., Ségonne, F., Fischl, B., Quinn, B., Dickerson, B., Blacker, D., Buckner, R., Dale, A., Maguire, R., Hyman, B., Albert, M. and Killiany, R. (2006). An automated labeling system for subdividing the human cerebral cortex on MRI scans into gyral based regions of interest. NeuroImage, 31(3), pp.968980.

Doeller, C., Barry, C. and Burgess, N. (2010). Evidence for grid cells in a human memory network. Nature, 463(7281), pp.657-661.

Ekstrom, A., Kahana, M., Caplan, J., Fields, T., Isham, E., Newman, E. and Fried, I. (2003). Cellular networks underlying human spatial navigation. Nature, 425(6954), pp.184-188.

Feigenbaum, J., Polkey, C. and Morris, R. (1996). Deficits in spatial working memory after unilateral temporal lobectomy in man. Neuropsychologia, 34(3), pp.163-176.

Ferrier, D. (1876). The functions of the brain. London: Smith, Elder.

Fischl, B., van der Kouwe, A., Destrieux, C., Halgren, E., Ségonne, F., Salat, D., Busa, E., Seidman, L., Goldstein, J., Kennedy, D., Caviness, V., Makris, N., Rosen, B. and Dale, A. (2004). Automatically Parcellating the Human Cerebral Cortex. Cerebral Cortex, 14(1), pp.11-22. 
Fischl, B. and Dale, A. (2000). Measuring the thickness of the human cerebral cortex from magnetic resonance images. Proceedings of the National Academy of Sciences, 97(20), pp.11050-11055.

Fischl, B., Liu, A. and Dale, A. (2001). Automated manifold surgery: constructing geometrically accurate and topologically correct models of the human cerebral cortex. IEEE Transactions on Medical Imaging, 20(1), pp.70-80.

Fischl, B., Salat, D., Busa, E., Albert, M., Dieterich, M., Haselgrove, C., van der Kouwe, A., Killiany, R., Kennedy, D., Klaveness, S., Montillo, A., Makris, N., Rosen, B. and Dale, A. (2002). Whole Brain Segmentation. Neuron, 33(3), pp.341-355.

Fischl, B., Salat, D., van der Kouwe, A., Makris, N., Ségonne, F., Quinn, B. and Dale, A. (2004). Sequenceindependent segmentation of magnetic resonance images. NeuroImage, 23, pp.S69-S84.

Fischl, B., Sereno, M., Tootell, R. and Dale, A. (1999). High-resolution intersubject averaging and a coordinate system for the cortical surface. Human Brain Mapping, 8(4), pp.272-284.

Frisk, V. and Milner, B. (1990). The role of the left hippocampal region in the acquisition and retention of story content. Neuropsychologia, 28(4), pp.349-359.

Friston, K., Ashburner, J., Kiebel, S., Nichols, T. and Penny, W. (2007). Statistical parametric mapping. Amsterdam: Elsevier/Academic Press.

Friston, K., Fletcher, P., Josephs, O., Holmes, A., Rugg, M. and Turner, R. (1998). Event-Related fMRI: Characterizing Differential Responses. NeuroImage, 7(1), pp.30-40.

Friston, K., Holmes, A., Worsley, K., Poline, J., Frith, C. and Frackowiak, R. (1994). Statistical parametric maps in functional imaging: A general linear approach. Human Brain Mapping, 2(4), pp.189-210.

Glover, G. (1999). Deconvolution of Impulse Response in Event-Related BOLD fMRI. NeuroImage, 9(4), pp.416-429.

Hafting, T., Fyhn, M., Molden, S., Moser, M. and Moser, E. (2005). Microstructure of a spatial map in the entorhinal cortex. Nature, 436(7052), pp.801-806.

Han, X., Jovicich, J., Salat, D., van der Kouwe, A., Quinn, B., Czanner, S., Busa, E., Pacheco, J., Albert, M., Killiany, R., Maguire, P., Rosas, D., Makris, N., Dale, A., Dickerson, B. and Fischl, B. (2006). Reliability of MRI-derived measurements of human cerebral cortical thickness: The effects of field strength, scanner upgrade and manufacturer. NeuroImage, 32(1), pp.180-194. 
Hirano, Y., Stefanovic, B. and Silva, A. (2011). Spatiotemporal Evolution of the Functional Magnetic Resonance Imaging Response to Ultrashort Stimuli. Journal of Neuroscience, 31(4), pp.1440-1447.

Iglesias, J., Augustinack, J., Nguyen, K., Player, C., Player, A., Wright, M., Roy, N., Frosch, M., McKee, A., Wald, L., Fischl, B. and Van Leemput, K. (2015). A computational atlas of the hippocampal formation using ex vivo, ultra-high resolution MRI: Application to adaptive segmentation of in vivo MRI. NeuroImage, 115, pp.117-137.

Jacobs, J., Weidemann, C., Miller, J., Solway, A., Burke, J., Wei, X., Suthana, N., Sperling, M., Sharan, A., Fried, I. and Kahana, M. (2013). Direct recordings of grid-like neuronal activity in human spatial navigation. Nature Neuroscience, 16(9), pp.1188-1190.

Jenkinson, M., Beckmann, C., Behrens, T., Woolrich, M. and Smith, S. (2012). FSL. NeuroImage, 62(2), pp.782-790.

Jezzard, P., Matthews, P. and Smith, S. (2001). Functional MRI. Oxford: Oxford University Press.

Kesner, R. and Martinez, J. (2007). Neurobiology of learning and memory. Amsterdam: Academic Press.

Kolb, B. and Whishaw, I. (1996). Fundamentals of human neuropsychology. 6th ed. New York: Freeman.

Lacy, J., Yassa, M., Stark, S., Muftuler, L. and Stark, C. (2010). Distinct pattern separation related transfer functions in human CA3/dentate and CA1 revealed using high-resolution fMRI and variable mnemonic similarity. Learning \& Memory, 18(1), pp.15-18.

Lauterbur, P. (1973). Image Formation by Induced Local Interactions: Examples Employing Nuclear Magnetic Resonance. Nature, 242(5394), pp.190-191.

Logothetis, N., Pauls, J., Augath, M., Trinath, T. and Oeltermann, A. (2001). Neurophysiological investigation of the basis of the fMRI signal. Nature, 412, pp.150-157.

Mahmoudi, A., Takerkart, S., Regragui, F., Boussaoud, D. and Brovelli, A. (2012). Multivoxel Pattern Analysis for fMRI Data: A Review. Computational and Mathematical Methods in Medicine, 2012, pp.1-14.

Malonek, D. and Grinvald, A. (1996). Interactions between Electrical Activity and Cortical Microcirculation Revealed by Imaging Spectroscopy: Implications for Functional Brain Mapping. Science, 272(5261), pp.551-554.

Mansfield, P. (1977). Multi-planar image formation using NMR spin echoes. J. Phys. C: Solid State Phys., 10(3), pp.L55-L58. 
Miller, J., Fried, I., Suthana, N. and Jacobs, J. (2015). Repeating Spatial Activations in Human Entorhinal Cortex. Current Biology, 25(8), pp.1080-1085.

Morris, J. (2005). Early-stage and preclinical Alzheimer disease. Alzheimer Disease and Associated Disorders, 19(3), pp.163-165.

Nocedal, J. and Wright, S. (2006). Numerical optimization. New York: Springer.

Ogawa, S., Lee, T., Kay, A. and Tank, D. (1990). Brain magnetic resonance imaging with contrast dependent on blood oxygenation. Proceedings of the National Academy of Sciences, 87(24), pp.98689872.

O'Keefe, J. and Dostrovsky, J. (1971). The hippocampus as a spatial map. Preliminary evidence from unit activity in the freely-moving rat. Brain Research, 34(1), pp.171-175.

O'Keefe, J. and Nadel, L. (1978). The hippocampus as a cognitive map. Oxford: Clarendon Press.

O'Keefe, J., Burgess, N., Donnett, J., Jeffery, K. and Maguire, E. (1998). Place cells, navigational accuracy, and the human hippocampus. Philosophical Transactions of the Royal Society B: Biological Sciences, 353(1373), pp.1333-1340.

Papez, J. (1937). A proposed mechanism of emotion. Archives of Neurology And Psychiatry, 38(4), p.725.

Pihlajamaki, M., Tanila, H., Kononen, M., Hanninen, T., Hamalainen, A., Soininen, H. and Aronen, H. (2004). Visual presentation of novel objects and new spatial arrangements of objects differentially activates the medial temporal lobe subareas in humans. European Journal of Neuroscience, 19(7), pp.1939-1949.

Ploner, C., Gaymard, B., Rivaud-Péchoux, S., Baulac, M., Clémenceau, S., Samson, S. and PierrotDeseilligny, C. (2000). Lesions Affecting the Parahippocampal Cortex Yield Spatial Memory Deficits in Humans. Cerebral Cortex, 10(12), pp.1211-1216.

Rajapakse, J., Kruggel, F., Maisog, J. and Yves von Cramon, D. (1998). Modeling hemodynamic response for analysis of functional MRI time-series. Human Brain Mapping, 6(4), pp.283-300.

Reuter, M., Rosas, H. and Fischl, B. (2010). Highly accurate inverse consistent registration: A robust approach. NeuroImage, 53(4), pp.1181-1196.

Reuter, M., Schmansky, N., Rosas, H. and Fischl, B. (2012). Within-subject template estimation for unbiased longitudinal image analysis. NeuroImage, 61(4), pp.1402-1418. 
Richter, W., Ugurbil, K. and Kim, S. (1996). Limitations of temporal resolution in fMRI.NeuroImage, 3(3), p.S38.

Salimi-Khorshidi, G., Douaud, G., Beckmann, C., Glasser, M., Griffanti, L. and Smith, S. (2014). Automatic denoising of functional MRI data: Combining independent component analysis and hierarchical fusion of classifiers. NeuroImage, 90, pp.449-468.

Sargolini, F., Fyhn, M., Hafting, T., McNaughton, B., Witter, M., Moser, M. and Moser, E. (2006). Conjunctive Representation of Position, Direction, and Velocity in Entorhinal Cortex. Science, 312(5774), pp.758-762.

Ségonne, F., Dale, A., Busa, E., Glessner, M., Salat, D., Hahn, H. and Fischl, B. (2004). A hybrid approach to the skull stripping problem in MRI. NeuroImage, 22(3), pp.1060-1075.

Segonne, F., Pacheco, J. and Fischl, B. (2007). Geometrically Accurate Topology-Correction of Cortical Surfaces Using Nonseparating Loops. IEEE Transactions on Medical Imaging, 26(4), pp.518-529.

Sled, J., Zijdenbos, A. and Evans, A. (1998). A nonparametric method for automatic correction of intensity nonuniformity in MRI data. IEEE Transactions on Medical Imaging, 17(1), pp.87-97.

Sloan, N. and Jasper, H. (1950). Studies of the regulatory functions of the limbic cortex.Electroencephalography and Clinical Neurophysiology, 2(1-4), pp.317-327.

Smith, M. and Milner, B. (1981). The role of the right hippocampus in the recall of spatial location.Neuropsychologia, 19(6), pp.781-793.

Smith, S., Miller, K., Moeller, S., Xu, J., Auerbach, E., Woolrich, M., Beckmann, C., Jenkinson, M., Andersson, J., Glasser, M., Van Essen, D., Feinberg, D., Yacoub, E. and Ugurbil, K. (2012). Temporally-independent functional modes of spontaneous brain activity. Proceedings of the National Academy of Sciences, 109(8), pp.3131-3136.

Sperling, R., Aisen, P., Beckett, L., Bennett, D., Craft, S., Fagan, A., Iwatsubo, T., Jack, C., Kaye, J., Montine, T., Park, D., Reiman, E., Rowe, C., Siemers, E., Stern, Y., Yaffe, K., Carrillo, M., Thies, B., Morrison-Bogorad, M., Wagster, M. and Phelps, C. (2011). Toward defining the preclinical stages of Alzheimer's disease: Recommendations from the National Institute on Aging-Alzheimer's Association workgroups on diagnostic guidelines for Alzheimer's disease. Alzheimer's \& Dementia, 7(3), pp.280-292.

Taube, J., Muller, R. and Ranck, J. (1990). Head direction cells recorded from postsubiculum in freely moving rats. I. Description and qualitative analysis. The Journal of Neuroscience, 10(2), pp.420-435. 
Wansapura, J., Holland, S., Dunn, R. and Ball, W. (1999). NMR relaxation times in the human brain at 3.0 tesla. J. Magn. Reson. Imaging, 9(4), pp.531-538.

West, M. (1993). Regionally specific loss of neurons in the aging human hippocampus.Neurobiology of Aging, 14(4), pp.287-293.

Zarahn, E., Aguirre, G. and D'Esposito, M. (1997). A Trial-Based Experimental Design for fMRI. NeuroImage, 6(2), pp.122-138. 